\title{
Poster 1
}

\section{Coil Occlusion of Giant Serpentine Middle Cerebral Artery Aneurysm}

Syed W Abbas, Stroke Fellow', Varun Naragum, Fellow Stroke², Mohamad AbdalKader, Fellow Radiology'2, Viken Babikian, Stroke Program Director ${ }^{2}$, Keith Davies, Neurosurgery ${ }^{2}$, Thanh Nguyen, Neurointerventionalist ${ }^{2}$

${ }^{1}$ Boston University Medical Center, Boston, Massachusetts, USA; ${ }^{2}$ Boston Medical Center, Boston, Massachusetts, USA

Introduction: Giant serpentine brain aneurysms on neuroimaging present as large ( $>25 \mathrm{~mm}$ ) partially thrombosed globoid mass lesions containing eccentric serpiginous vascular channels with multiple entry and exit points (Coanda effect). Their clinical presentation is usually due to mass effect, surrounding edema and herniation. Commonly affected arteries include Middle Cerebral Artery, Vertebral artery and Posterior Cerebral Artery. Parent artery occlusion with endovascular coils alone or in combination with surgical bypass has been used in the past to manage such complicated vascular lesions.

Methods: Case-report of a patient presenting with giant MCA serpentine aneurysm

Results: A 60-year female presented for evaluation of chronic daily headaches complicated by gait difficulty, left hand weakness and cognitive slowing. Initial examination showed cognitive impairment, left face and arm weakness and bilateral extensor plantar response. A non-contrast CT head showed a heterogeneously calcified $5 \times 5.3 \times 5 \mathrm{~cm}$ right frontotemporal mass lesion with mid-line shift. MRI brain gradient-echo sequence showed laminated hemosiderin deposition inside the thrombus, postcontrast peripheral enhancement and mass effect. Immediately after the admission, her rapidly deteriorating neurological status prompted urgent intervention. Cerebral angiogram showed a $12-\mathrm{cm}$ long right MCA giant serpentine dissecting aneurysm. Surgical bypass or clipping were deemed technically inaccessible because of the extensive thrombus surrounding the aneurysm and significant mass effect. Balloon test occlusion followed by occlusion of the dysplastic MCA segment by endovascular coiling was chosen for treatment of the aneurysm. Follow up CT head showed complete obliteration of the aneurysm. It was complicated by malignant MCA ischemic stroke requiring urgent decompression hemicraniectomy. After rehabilitation, she showed recovery with residual left side hemiplegia. 
Conclusions: Our patient showed a complicated course following endovascular coil occlusion which was done in the setting of severe mass effect and worsening clinical condition of the patient. We recommend a case-based approach in such complicated patients.

Keywords: Aneurysm, Aneurysm Embolization, Coiling, Endovascular Financial Disclosures: The authors had no disclosures.

Grant Support: None. 


\section{Poster 2}

\section{Unique Variant of Idiopathic Intracranial Hypertension Dural Sinus Stenting}

Michael G Abraham, MD, FAHA

${ }^{1}$ University of Kansas Medical Center, Kansas CIty, Kansas, USA

Introduction: Endovascular dural sinus stenting is being performed with increased frequency for idiopathic intracranial hypertension (IIH). This case represents a unique variant of dominant dural sinus drainage. A 55 year-old female with IIH presented with decreased vision and papilledema. Two lumbar punctures demonstrated opening pressures of 31 and $38 \mathrm{cmH} 2 \mathrm{O}$.

Methods: Cerebral venography demonstrated hypoplastic bilateral transverse-sigmoid sinuses with dominant drainage through a persistent occipitalmarginal sinus (POMS). Multiple enlarged arachnoid granulations resulted in stenosis in the marginal sinus (85\%) with an $18 \mathrm{mmHg}$ mean pressure gradient across the stenosis. The decision was made to stent the POMS. Under general anesthesia a 6-French $90 \mathrm{~cm}$ Shuttle sheath (Cook Medical, Bloomington, IN) was placed in the right common femoral vein. A $105 \mathrm{~cm}$ 6-French 0.071 Benchmark guide catheter (Penumbra Inc., Alameda, CA) was advanced over its inner catheter over a $180 \mathrm{~cm} 0.035$ Glide wire (Terumo Interventional Systems, Somerset, NJ) into the right jugular bulb. The guide catheter was advanced into the superior POMS. A 7 x $40 \mathrm{~mm}$ Precise stent (Cordis, Milpitas, CA) was deployed with significant residual stenosis in the inferior portion of the stent. A Sterling $6 \times 20 \mathrm{~mm}$ balloon (Boston Scientific, Marlborough, MA) was prepped with 70:30 contrast:saline and angioplasty was performed to 6 ATM with resolution of the stenosis. Additional coverage was required below the stent; therefore, a 7 x $30 \mathrm{~mm}$ Precise stent was deployed into the persistent marginal sinus, overlapping the first stent with residual stenosis in the inferior portion of the stent. Angioplasty resulted in resolution of the stenosis.

Results: A final superior sagittal sinus venogram demonstrated resolution of the POMS stenosis with flow only into the POMS. Six-month follow-up venography demonstrated continued stent patency and clinical symptoms remained resolved.

Conclusions: Recognizing the dominant dural sinus drainage route is critical for proper treatment in IIH.

Keywords: Intracranial Stenosis Stenting And Angioplasty, Stenting, Endovascular, New Technique, Angioplasty

Financial Disclosures: Stryker Neurovascular - Consultant; Boehringer Ingelheim - Speaker's Bureau

Grant Support: None. 


\section{Poster 3}

\section{Subclavian Steal Syndrome Secondary to Dialysis AVF Treated with Covered Biliary Stent}

Shashank Agarwal, $M D^{1}$, Patrick Kwon, $M D^{1}$, Jeffrey Farkas, $M D^{1}$, George Selas, $M D^{1}$, Karthikeyan Arcot, $M D^{1}$, Ambooj Tiwari, $M D^{1}$, Lisa Schwartz, $M D^{1}$

${ }^{1}$ NYU Langone Health, New York, New York, USA

Introduction: Subclavian steal syndrome (SSS) is usually due to stenosis of the Subclavian Artery (SA) proximal to the origin of the vertebral artery (VA). SSS with intact SA in patients with dialysis arterio-venous fistulas (AVF) has been occasionally reported[1-4]. We present a unique case of the same successfully treated with a covered stent.

Methods: A 65-year-old male with DM, HTN, ESRD and left brachial artery- basilic vein fistula $(2.27 \mathrm{~L} / \mathrm{min}$ flow) had multiple admissions with vertebrobasilar symptoms in the setting of elevated BP. Repeated evaluations with CT/CTA/MRI were negative for steno-occlusive disease or infarction. Therefore, symptoms were attributed to hypertensive urgency. On his third presentation in 4 months, he had additional symptoms of left arm pain, weakness and numbness. Signs included left arm hyperemia, warmth and mild motor-sensory deficits.

Results: MRA demonstrated reverse flow of blood in left VA with focal stenosis of proximal left SA. Angiogram showed a kinked LSA and rapid/ early shunting to the subcalvian vein. There was no ante-grade visualization of the LVA with reverse flow from the RVA via VB junction. However on compression of the shunt with a BP cuff, antegrade flow in LVA reappeared with disappearance of RVA-LVA steal. BP transduction revealed a $50-\mathrm{mmHg}$ point difference across the kinked segment. Subsequently, proximal left SA was stented with a covered biliary stent resulting in disappearance in RVALVA \& LSA-LSV shunts, reappearance of antegrade LVA flow and resolution of symptoms.

Conclusions: High-flow AVF is an underdiagnosed cause of symptomatic SSS. We suggest determining AVF flow speeds in any hemodialysis patient who presents with symptoms of posterior circulation insufficiency and obtaining noninvasive vascular studies if flow exceeds $2 \mathrm{~L} / \mathrm{min}$ or if there has been a recent increase in measured flow during hemodialysis. Obtaining vascular studies with and without fistula compression could be of additional diagnostic utility.

Keywords: Vascular Imaging, Interventional Neuroradiology, Diagnostic Neuroradiology

Financial Disclosures: The authors had no disclosures.

Grant Support: None. 


\title{
Poster 4
}

\section{Case Report and Literature Review: Balloon Thrombectomy for Cerebral Venous Thrombosis with an Atypical Headache}

\author{
Anthony Alvarado-Ortiz, DO' ${ }^{1}$, Jason Nazar, DO', Michelle Evans, DO'2, Ritesh \\ Kaushal, $M D^{3}$, Jill Liebman, $D O^{2}$ \\ 'Larkin Community Hospital, Palmetto General Hospital, Miami, Florida, USA; \\ ${ }^{2}$ Larkin Community Hospital, Miami, Florida, USA; ${ }^{3}$ Palmetto General Hospital, \\ Miami, Florida, USA
}

Introduction: Cerebral venous thrombosis [CVT] ranges in incidence of 1.32 per 100,000 population.1. Often, CVT symptoms can present with comorbid headache [HA] and severe pain in isolation of other signs and symptoms, which substantiates the necessity of addressing a robust differential for new-onset HA. Furthermore, use of neurointerventional has been considered in some cases of CVT, however evidence based outcomes comparing best medical practice versus emergent endovascular treatment is limited to case studies and anecdotical clinical judgment.

Methods: A case study was performed which included detailing a patient suffering from a severe CVT, who presented with an atypical ahead, and was successfully treated with balloon thrombectomy. Also, a thorough literature review on Pubmed was completed, including search parameters "Cerebral Venous Thrombosis and thrombectomy."

Results: After anticoagulation and acute clot disruption with a balloon catheter the patient returned to baseline during two weeks of hospitalization, including immediate improvement of HA. So, this case highlights the importance of timely and methodically evaluating a robust differential for newonset HA. And then, specific prompting: in this case it was revealed the patient was involved in a motor-vehicle-accident two weeks prior to admission, which may have incited thrombosis in the context of hypercoagulability secondary to oral contraceptive use. Lastly, the case documents an efficacious, novel treatment modality for cerebral venous thrombosis: mechanical balloon thrombectomy.

Conclusions: Currently, the literature is limited to case studies/series, including sparse discussion of specific hardware and technique. There aren't controlled endovascular studies in the treatment of CVT; no well-powered studies defining utility or comparing the efficacy in each of these techniques for specific amiable populations. A balloon catheter is an advantageous hardware choice for mechanical disruption, because thrombolytic agents can be injected via the inner lumen with the balloon inflated.

Keywords: Cerebral Sinus Thrombosis Therapy, Cerebral Sinus And Venous Thrombosis, Balloon Angioplasty, Endovascular Therapy, Neurointerventional Education

Financial Disclosures: The authors had no disclosures.

Grant Support: None. 


\title{
Poster 5
}

\section{Direct Occipital Artery Cutdown for Onyx Embolization of Refractory Dural Arteriovenous Fistula}

\author{
Aashish Anand, $M D^{1}$, Naregnia Pierre-Louis, $M D^{1}$, Parita Bhuva, $M D^{1}$, \\ Albert Yoo, $M D^{1}$ \\ ${ }^{1}$ Texas Stroke Institute, Plano, Texas, USA
}

Introduction: Endovascular treatment of dural arteriovenous fistulas (DAVF) can be limited by difficult trans-arterial access due to vessel tortuosity. We describe a case of DAVF refractory to traditional trans-arterial access, and where endovascular cure was achieved after direct cutdown to a markedly tortuous arterial feeder.

Methods: Case-report

Results: A 60-year-old man presented with right hemianopia. An angiogram revealed a Cognard Type IV DAVF with drainage directly into ectatic cortical veins in the left parieto-occipital region. Arterial feeders were from bilateral middle meningeal (MMA) and right occipital arteries (OA). There was a second, smaller Cognard Type III DAVF in the leaflet of left transverse sinus with mixed direct drainage into a dilated cortical vein and transverse sinus, and arterial feeders from left OA. Angiographic occlusion of both DAVFs was achieved after staged Onyx embolization of the arterial feeders. There was interval resolution of visual symptoms. A follow-up angiogram revealed recurrence of the Cognard Type IV left occipital DAVF with recanalization of arterial feeders from petrosquamosal branch of left MMA and a trans-osseous branch of right OA. Attempts at achieving optimal microcatheter position for Onyx embolization of these arterial feeders were unsuccessful due to extreme vessel tortuosity. A successful surgical cutdown of the distal right $\mathrm{OA}$ was achieved after angiographic localization of the optimal cutdown site. The right OA was transected and the distal segment was directly cannulated with a DMSO-compatible balloon microcatheter. A stay suture around distal OA segment at the cannulated segment of microcatheter mitigated Onyx reflux, and facilitated antegrade Onyx penetration through the trans-osseous branch into the nidus of DAVF and proximal draining vein. A complete angiographic obliteration of the recurrent DAVF was achieved.

Conclusions: A direct cutdown to distal arterial feeders is a feasible and potentially safe option for endovascular treatment of DAVF complicated by difficult trans-arterial access.

Keywords: Avm Embolization, Intracerebral Hemorrhage, Cerebral Arteriovenous Malformations, Onyx

Financial Disclosures: The authors had no disclosures.

Grant Support: None. 


\title{
Poster 6
}

\section{Reversible Cerebral Vasoconstriction Syndrome (RCVS) Triggered by Aseptic Meningitis}

\author{
Yogesh Gujrati, Vascular Neurology Fellow', Anmar Razak, NIS Fellowship \\ Director ${ }^{1}$, Michael DeGeorgia, Professor of Neurology ${ }^{2}$ \\ ${ }^{1}$ Michigan State University Department of Neurology and Ophthalmology, \\ East Lansing, Michigan, USA; ${ }^{2}$ University Hospitals Cleveland Medical Center, \\ Cleveland, Ohio, USA
}

Introduction: RCVS is characterized by severe headaches, with or without acute neurological symptoms, and diffuse segmental constriction of cerebral arteries that resolve spontaneously within 3 months. CSF pleocytosis is generally not seen in RCVS.

Methods: Case report and literature pertinent to RCVS and aseptic meningitis was reviewed.

Results: A 47-year old woman developed acute headache. Head CT and CTA were normal. LP revealed CSF pleocytosis (30 WBCs, $85 \%$ lymphocytes) and mildly elevated protein. She was diagnosed with aseptic meningitis and treated initially with intravenous acyclovir. CSF cultures and HSV PCR were negative. She was discharged home but continued to have headache, nausea and vomiting. Ten days later, she developed right hemiparesis and confusion. Brain MRI showed acute, bilateral watershed territory hemispheric infarcts. Intracranial MRA showed diffuse and multifocal vascular narrowing. Intravenous methylprednisolone was started initially for possible CNS vasculitis. She developed worsening right hemiparesis associated with low blood pressure. Repeat brain MRI showed enlargement of the previous watershed territory infarcts and a new left cerebellar infarct. Following blood pressure augmentation, her exam stabilized. Repeat LP showed persistent but improving CSF pleocytosis (7 WBCs). Cytology showed no evidence of malignancy and VZV PCR was negative. Rheumatologic work up revealed no evidence of autoimmune disease. Catheter angiography revealed widespread and diffuse vasoconstriction that responded dramatically to intraarterial injection of verapamil. She was started on oral Verapamil, improved clinically, and was discharged to acute rehabilitation.

Conclusions: RCVS is a clinical diagnosis. CSF pleocytosis is very uncommon occurring in only $3-8 \%$ of patients. Our patient presented with aseptic meningitis and had normal vasculature initially only to develop severe vasoconstriction 10 days later. This suggests that RCVS may, in some cases, be triggered by aseptic meningitis.

Keywords: Acute Stroke, Angiogram, Cns Vasculitis, MRA, Cerebrovascular Disease

Financial Disclosures: The authors had no disclosures.

Grant Support: None. 


\section{Poster 7}

\section{Stroke Intervention During Hurricane Irma Without CT or MRI}

Ritesh Kaushal, $M D^{1}$, Wilson Cueva, $M D^{1}$, Ali Malek, $M D^{1}$, Jose Gomez, $R T^{2}$, Karan Gupta, $M D^{3}$, Nils Mueller-Kronast, $M D^{1}$

${ }^{1}$ Advanced Neuroscience Network, Tenet Florida, Hialeah, Florida, USA;

${ }^{2}$ Palmetto, Hialeah, Florida, USA; ${ }^{3}$ Palmetto Hospital, Hialeah, Florida, USA

Introduction: Hurricane Irma tore through South Florida early September 2017. It had significant impact on health care management services. The neurointerventional services at our hospital in South Florida stayed in-house during the storm.

Methods: 64 year old male, a supervisor for Florida Gas and Light (FPL) was brought by Miami-Dade EMS in the waning period of the hurricane. Patient was at work when he suddenly developed right-side weakness and aphasia. The EMS personnel were working close-by so they immediately brought him to our comprehensive stroke center within 40 mins of symptom onset. NIHSS on arrival 16. Patient was on Xarelto for history of DVT/PE. 30 mins prior to his arrival, all three of our CT scanners and MRI went down, likely secondary to the numerous switches due to power failure and generators kicking in. Any transfers were not possible as EMS was not at normal functioning due to the ongoing storm. With limited options, we did a DynaCT on our Seimens Artis biplane. We were able to rule out a hemorrhage on the DynaCT and then we proceeded with endovascular therapy.

Results: Patient was found to have a left MCA M1 occlusion. Mechanical embolectomy using Solitaire Platinum $6 \times 40$ was performed. TICI score at end of procedure was 3 . Door-to-groin time 27 mins. Groin-to-recanalization time 25 mins. Symptom onset-recanalization time 92 mins. The following morning his NIHSS was 0 . He was discharged two days later with no neurological deficits.

Conclusions: In emergent situations, if basic imaging tools like CTs and MRIs are not operational, DynaCT in the angio suite could be a useful modality to rule out hemorrhage in hyperacute strokes. Endovascular therapy can then be offered. Further studies can also look at risk-benefit of direct DynaCT in hyperacute strokes towards significantly lowering the door-groin time.

Keywords: Endovascular Therapy, Stroke, Imaging

Financial Disclosures: The authors had no disclosures.

Grant Support: None. 


\section{Poster 8}

\section{Cerebral Infarction Due to Thrombosis Transverse Sinus}

Aida Kondybayeva, $\mathrm{MD}^{1}$, Saltanat Kamenova, Professor ${ }^{1}$, Murat Zhanuzakov, Professor $^{2}$

${ }^{1}$ Asfendiyarov Kazakh National Medical University, Almaty, Kazakhstan;

${ }^{2}$ Kazakh Medical University of Continuing Education, Almaty, Kazakhstan

Introduction: Acute disturbance of cerebral circulation due to thrombosis of venous sinuses of the brain is a rare cause of cerebrovascular pathology. According to the International Studyon Cerebral Vein and Dural Sinus Thrombosis (2004), the incidence of venous stroke in adults is 3-4 cases per 1 million population annually. Risk factors for venous stroke are: severe dehydration, pregnancy, heart disease, nephrotic syndrome, coagulopathy, antiphospholipid syndrome, use of antifibrinolytic drugs, oral contraceptives, etc.

Methods: Clinical material was the observation of patient A., 35 years old, with thrombosis of the transverse sinus. Of the stroke risk factors take into account hypertension, pregnancy, childbirth and the antiphospholipid syndrome. From anamnesis: 6 days ago she gave birth to a healthy child, childbirth was physiological. Suddenly in the morning, after a dream, there was an acute headache in the occipital region, which later gradually grew. There was nausea and three times vomiting, which did not bring relief. The acute development of cerebral and focal symptoms indicated the development of acute cerebrovascular accident, one could think of the development of ischemic or hemorrhagic stroke. The character and severity of the headache in combination with local manifestations in the form of soft tissue edema in the mastoid process, pain during chewing and turnings of the head caused difficulties in the diagnosis of the etiology of the disease. Methods of neuroimaging, MRI of the brain, MRI angiography with contrasting allowed to diagnose venous thrombosis with the development of a stroke.

Results: A diagnosis was made: Cerebral infarction caused by thrombosis of the brain veins, nonpyogenic with hypertension. An anticoagulant, antibacterial, desensitizing and restorative therapy was performed. Against the background of treatment, the patient's condition in the dynamics improved.

Conclusions: Pregnancy and, especially, the postpartum period - the time of increased probability of sinus thrombosis in risk groups.

Keywords: Acute Stroke, Cerebral Sinus And Venous Thrombosis, Cerebrovascular Disease, Ischemic Stroke, Stroke

Financial Disclosures: The authors had no disclosures.

Grant Support: None. 


\title{
Poster 9
}

\section{Acute Dystonia in the Setting of Recanalized Left MCA with No Residual Neurological Deficits}

\author{
Rebecca Lalchan, $D 0^{1}$, Ryan Bo, $M D^{2}$, Jeremy $M$ Liff, $M D^{2}$, Jennifer Frontera, $M D^{1}$, \\ Patrick Kwon, $M D^{1}$, Ambooj Tiwari, MPH, MD ${ }^{2}$
}

${ }^{1}$ NYU Langone Hospital Brooklyn, Brooklyn, New York, USA; ${ }^{2}$ NYU Langone Hospital Brooklyn Interventional Neuro Associates, Brooklyn, New York, USA

Introduction: Adult onset focal dystonia often affects the upper extremities and cervical region but less often the lower extremities. Dystonia is the second most reported movement disorder post-stroke and often has a delayed presentation ranging from weeks to months. Most reports are in cases where there is permanent and substantial tissue injury. The clinical significance of Basal ganglia infarction or petechial hemorrhage following endovascular therapy for MCA occlusion is not well understood. The development of dystonia in the setting of a recanalized LMCA has never been reported before.

Methods: A 39-year-old female presented with Left MCA occlusion. She had no other medical history except for an idiopathic left basal ganglia hemorrhagic stroke 6 months ago with residual mild forearm weakness. She underwent urgent mechanical thrombectomy with successful sequential recanalization of her inferior followed by superior division. The latter was complicated by a mild self-limiting subarachnoid hemorrhage in the left sylvian fissure. She had small petechial hemorrhages in the left basal ganglia on MRI. However, she recovered completely in 5 days and was discharged with a NIHSS of 1 (similar to baseline) as well as mRS of 2 . Ten months later, she developed a painful, fixed right lower extremity dystonia where her ankle was inverted and plantarflexed with her toes curled. This was treated with multiple anticholinergic \& GABAergic medications as well as Botox to only achieve partial success. Currently DBS is also being considered.

Results: This presentation is unique and it is possible that her delayed presentation of dystonia demonstrates the abnormal plasticity of the basal ganglia network especially following multiple insults (hemorrhagic followed by ischemic). This may result in hyperactivity of the regulatory motor tone pathways without significant cortical insult.

Conclusions: The true extent of mild basal ganglia injury in context of MCA recanalization is not completely understood.

Keywords: MCA, Endovascular Therapy, Acute Ischemic Stroke Intervention

Financial Disclosures: The authors had no disclosures.

Grant Support: None. 
Poster 10

Abstract withdrawn 


\section{Poster 11}

\section{Spontaneous Internal Carotid Artery Dissections Associated with the Use of Phentermine, a Weight Loss Supplement}

Richard A Lewis, M.D. ${ }^{1}$, Vasu Saini, M.D. ', Nicholas Liaw, M.D. Ph.D. ', Sebastian

Koch, M.D. ${ }^{1}$

'University of Miami/Jackson Memorial Hospital, Miami, Florida, USA

Introduction: Phentermine is a centrally-acting amphetamine-related sympathomimetic anorectic, used as a short-term (few weeks) adjunctive treatment of obesity. Previous reports link the use of Phentermine, in combination with Fenfluramine ("Fen-Phen"), with the development of valvular heart disease. We report a case of bilateral spontaneous internal carotid artery (ICA) dissections linked with the prolonged use of Phentermine.

Methods: A case report study and review of the literature regarding spontaneous ICA dissections and the use of Phentermine.

Results: A 50 year-old right-handed Hispanic female with history of hypertension and migraine headaches, presented with a 2-week history of headache and neck pain, associated with the sudden-onset slurred speech and right-sided weakness. She denied head or neck trauma. Imaging revealed bilateral ICA dissections from origin of the ICAs to the supraclinoid segments with complete occlusion to the left ICA and "trickle flow" through the right ICA, with left fetal circulation leading to hypoperfusion and left ACAMCA border-zone territorial infarction. The patient was anticoagulated with intravenous Heparin, and eventually underwent endovascular stenting of the right ICA and started on dual antiplatelet therapy. On review of the patient's medications, she had been taking Phentermine $37.5 \mathrm{mg}$ daily for over 1 year for weight loss. Work-up for the cause of the dissections was largely negative. In a similar study, undertaken by Orzechowski et al. at the Mayo Clinic in 2006, a 37 year-old woman was found to have bilateral ICA dissections associated with the use of Phentermine as well as Pseudoephedrine for allergic rhinitis.

Conclusions: In conclusion, care must be taken with prolonged use of weight loss supplements, particularly those with sympathomimetic qualities, as they may cause severe vascular abnormalities, such as spontaneous ICA dissections.

Keywords: Carotid Stenting And Angioplasty, Acute Ischemic Stroke Intervention, Cerebrovascular Disease, Acute Stroke, Stenting

Financial Disclosures: The authors had no disclosures.

Grant Support: None. 


\section{Poster 12}

\section{Case Report: Pipeline and Coil Embolization of a Previously Ruptured and Coiled Dissecting P2 Aneurysm}

Conrad W Liang, $M D, P h D^{1}$, Mazen Noufal, $M D^{1}$, Vaninder S Chhabra, $M D^{1}$, Todd

$M$ Goldenberg, $M D^{1}$

${ }^{1}$ Kaiser Permanente, Fontana Medical Center, Fontana, California, USA

Introduction: Posterior cerebral artery aneurysms are relatively uncommon and the etiology and management of these vascular lesions remains uncertain. We present a case of a small, wide-necked ruptured P2 aneurysm which was initially treated with primary coil embolization. The lesion demonstrated substantial growth on follow up and was subsequently treated successfully with Pipeline plus coil embolization.

Methods: A 57 year old patient presented with $\mathrm{HH} 3 / \mathrm{F} 4 \mathrm{SAH}$ and was found to have a wide-necked, $3 \mathrm{~mm}$ left P2 aneurysm. Coil embolization was decided as the initial management strategy to avoid the need for antiplatelet therapy in the setting of acute rupture and ventriculostomy placement. Follow up angiogram 1 week after admission demonstrated persistent occlusion of the aneurysm. The patient was discharged to SNF after 24 days in the hospital. Over the next several months she made a gradual clinical recovery to modified Rankin 1 and returned for follow up at 6 months. Angiogram demonstrated substantial enlargement of the previously treated aneurysm to $9.6 \mathrm{~mm}$, with the previous coil mass only partially within the opacifying portion of the aneurysm.

Results: Repeat coil embolization was performed urgently to protect the aneurysm. The patient was then placed on aspirin and Plavix and 3 days later a Pipeline stent was placed along the neck of the aneurysm from the posterior communicating artery to the P3 segment. She did well and was discharged from the hospital the following day with no neurologic deficits.

Conclusions: Ruptured and presumed dissecting PCA aneurysms may be treated by primary coiling in the acute phase to protect the patient from rerupture, but close follow up is needed as these aneurysms may be unstable and demonstrate considerable growth over a short period of time. If growth occurs, flow diversion may be the best approach to prevent aneurysm regrowth and re-rupture.

Keywords: Aneurysm Embolization, Flow Diverter, Coiling, Decision Analysis

Financial Disclosures: The authors had no disclosures.

Grant Support: None. 


\title{
Poster 13
}

\section{Carotid Artery Web Causing Recurrent Middle Cerebral Artery Acute Ischemic Strokes in a Young Patient}

\author{
Sishir Mannava, M.D. ${ }^{1}$, Amer M Malik, $M D^{1}$, Sebastian Koch, $M D^{1}$, Dileep $R$ \\ Yavagal, $M D^{2}$ \\ 'University of Miami Miller School of Medicine, Miami, Florida, USA; \\ 2University of Miami, Miami, Florida, USA
}

Introduction: Carotid artery webs (CAW) are intraluminal shelf-like filling defects at the carotid bulb. CAWs have been investigated as a potential etiology of acute ischemic stroke (AIS), especially in young stroke populations.

Methods: A 43 year-old African-American female with hyperlipidemia suffered two recurrent right middle cerebral artery (MCA) AIS over a fouryear span. She received acute systemic thrombolysis for one of these presentations. Stroke etiology remained cryptogenic despite comprehensive workup that included prolonged cardiac monitoring and negative thrombophilia workup. Associated diagnoses included complicated migraine headaches and non-convulsive seizures. After the second AIS event, concern existed for extracranial right internal carotid artery (ICA) disease due to presence of a flap on cerebral digital subtraction angiogram (DSA) at an external institution not entirely consistent with dissection. Patient was treated with dual antiplatelet therapy for 3 months and then converted to clopidogrel monotherapy. She was referred to our tertiary institution. Follow-up computed tomography angiogram imaging continued to demonstrate proximal right ICA carotid vault dissection/intimal flap with bilateral proximal ICA fusiform aneurysmal dilatation concerning for possible fibromuscular dysplasia (FMD). Follow-up cerebral DSA in December 2016 showed no evidence of FMD and she was continued on clopidogrel monotherapy and statin. In September 2017, she experienced a new small right MCA AIS while faithfully taking her medication regimen.

Results: She underwent repeat cerebral DSA and was confirmed to have a right CAW that was successfully treated with carotid stent via endovascular approach.

Conclusions: We present a case of a young patient confirmed to have CAW causing recurrent AIS in a distribution ipsilateral to the CAW. This case report is important because it identifies an under-recognized etiology of AIS in young patients that can commonly be underappreciated on imaging. More rigor should be utilized when evaluating imaging for CAW as a potential cause of AIS.

Keywords: Carotid Stenting And Angioplasty, Cerebrovascular Disease, Interventional Neuroradiology, Stroke, Carotid

Financial Disclosures: The authors had no disclosures.

Grant Support: None. 


\title{
Poster 14
}

\section{Left Internal Carotid Artery Hypoplasia and Direct Aortic Arch Origin: Case Report and Embryology Review}

\author{
John J McGlynn, First author ', Atif Naveed, Second author', Hesham Masoud, \\ Third author ${ }^{1}$ \\ 'SUNY Upstate Medical University, Syracuse, New York, USA
}

Introduction: We present a case of a patient with rare variant anatomy, a hypoplastic left internal carotid artery (L-ICA) originating directly from the aortic arch. This rare congenital anomaly should be looked out for on imaging in acute stroke cases as it has distinct radiographic signs and can have important clinical implications.

Methods: Internal carotid artery (ICA) hypoplasia and agenesis are quite rare, with an estimated combined prevalence of $0.01 \%$. These anomalies are usually discovered incidentally, and are typically asymptomatic. This asymptomatic nature is due to the development of collateral circulatory routes. Despite this, there is still associated morbidity and mortality, as affected individuals have significantly increased risk for cerebral aneurysms. These anomalies can be readily identified on computed tomography; a narrow or absent carotid canal is indicative of ICA hypoplasia or agenesis respectively.

Results: A 69-year-old woman presented to a community emergency department with sudden-onset right hemiparesis. Initial computed tomography angiography (CTA) read at an outside hospital reported complete occlusion of the L-ICA, and the patient was transferred to our center for endovascular treatment of presumed emergent large vessel occlusion. On arrival, the CTA images were reviewed and disclosed direct aortic arch origin and congenital hypoplasia of the L-ICA, and separate arch origin of the left external carotid artery.

Conclusions: In the setting of acute hemispheric motor deficit, a congenitally hypoplastic ICA may be initially interpreted as vessel occlusion or dissection with luminal narrowing, resulting in referral to endovascular-capable centers for management. This case report serves to explain the embryology behind this rare anatomic variant and emphasise the importance of reviewing the aortic arch and ipsilateral carotid canal, to distinguish between congenital anatomic variance and acute cerebrovascular pathology.

Keywords: Ischemic Stroke, Angiographic Ct, Imaging, Diagnostic Neuroradiology, Cerebrovascular Disease

Financial Disclosures: The authors had no disclosures.

Grant Support: None. 
Poster 15

\title{
Pipeline Flow Diversion in Failed Stent-Assisted Repair of Recurrent Dissecting Intracranial Pseudoaneurysm - A Case Report
}

\author{
Varun V Reddy, $M D^{1}$, Sahmaelah Javed, $M D^{2}$, Waleed Elnour, $M D^{2}$, Sumeet \\ Multani, MD2, Tony Wong, MD, Ph. $D^{3}$, Scott Brehaut, MD', Yahia M Lodi, MD, \\ FAHA, FANA, FAAN ${ }^{5}$
}

${ }^{1}$ UHS-WMC/Upstate Medical University, Binghamton/MVHS-Health, Johnson

City, New York, USA ; ${ }^{2}$ UHS-Wilson Medical Center/Upstate Medical University, Johnson City, New York, USA; ${ }^{3}$ UHS-Wilson Medical Center/Upstate Medical

University/MVHS-Health, Johnson City, New York, USA; ${ }^{4}$ MVHS-Health, Utica,

Utica, New York, USA; ${ }^{5}$ Upstate Medical University, Binghamton/WHS-Wilson

Medical Center/MVHS-Health, Binghamton, New York, USA

Introduction: The use of flow-diverter after stent-assisted repair of symptomatic dissecting pseudo-aneurysm (SDPA) is not described. Objective is to describe the technical and clinical outcome of three patients who underwent rescue Pipeline flow diversion to repair their recurrent SDPA.

Methods: Retrospective review.

Results: The first case is a 35-year-old woman who underwent stentassisted repair of 9-mm right petro-cavernous SDPA. Symptoms recurred and angiogram demonstrate aneurysm recurrence; a second intracranial stent was used and aneurysm was recoiled resulting in aneurysm obliteration and resolution of symptoms. Six-month angiogram revealed recurrence of $11 \mathrm{~mm}$ SDPA. A pipeline flow diversion was considered. Patient underwent uneventful placement of a pipeline device under conscious sedation and symptoms resolved. Follow-up angiogram confirmed aneurysm obliteration and normal remodeling of the artery. The second case was a 31-year-old women, required emergent stent-assisted coiling for her SDPA in left para-ophthalmic region with complete recovery. Symptoms recurred in 3 months and underwent coiling and placement of a second stent leading to occlusion of aneurysm and became asymptomatic. However, symptoms returned in 6 weeks due to recurrence of $7 \mathrm{~mm}$ aneurysm which was repaired with a pipeline flow diverter and symptoms resolved. Angiograms confirmed persistent obliteration. The 3rd case was a 76-year-old woman who required urgent stent-assisted repair of her 43-mm left ophthalmic SDPA with partial obliteration. Aneurysm recurred in 4 weeks, repaired with stent-coils and remained symptomatic. Underwent pipeline flow diversion; navigation of pipeline delivery catheter required a stiffer wire exchange and uneventful delivery of a long pipeline device. Symptoms resolved immediately and aneurysm was obliterated in 6 months.

Conclusions: Our series demonstrated that pipeline flow diversion could be used in recurrent SDPA that were treated with intracranial stent with good radio-graphical and clinical outcome. Further studies are required.

Keywords: Aneurysm Embolization, Pipeline, Flow Diverter, Stent Assisted, Coiling

Financial Disclosures: Speaker for Xarelto

Grant Support: None. 


\section{Poster 16}

\section{Traumatic Vertebral Arteriovenous Fistula with Stroke as Presenting Symptom as Well as Steal Phenomenon}

Daniel Ro, $M D^{1}$, Steve Cordina, $M D^{1}$, Markus Lammle, $M D^{1}$

${ }^{1}$ University of South Alabama, Mobile, Alabama, USA

Introduction: Vertebral arteriovenous fistula (AVF), defined as abnormal direct communication between the vertebral artery and the neighboring venous system, is a rare clinical entity with severe consequences if left untreated.

Methods: Case Report

Results: A 22-year-old man with penetrating neck injury presented with decreased level of consciousness as well as severe dysmetria, left greater than right. Non-contrast CT of the brain showed an extensive left cerebellar infarct in evolution. In addition, CT angiography of the head/neck revealed a nondisplaced fracture of the left transverse process at C6 level with contrast present in venous plexus within the transverse foramen. Subsequently, patient underwent diagnostic cerebral angiography, which revealed a steal phenomenon with retrograde flow to the distal basilar artery and the posterior cerebral arteries being supplied via bilateral posterior communicating arteries. Further investigation led to the discovery of left vertebral artery V2 segmentleft vertebral venous plexus fistula as the etiology. This was treated using an endovascular approach with radiographic cure. On the day of the discharge, the only deficit continued to be dysmetria, and patient was able to ambulate without assistance.

Conclusions: This traumatic injury likely caused a thromboembolic event resulting in acute ischemic infarction of cerebellum and formation of the arteriovenous fistula, resulting in a steal phenomenon seen later on cerebral angiography. Vertebral arteriovenous fistula should be considered in patients presenting with traumatic neck injury, and careful monitoring for stroke is warranted.

Keywords: Angiogram, Acute Stroke, Vertebral, Basilar, Embolization Financial Disclosures: The authors had no disclosures.

Grant Support: None. 


\title{
DIC Presenting with Diffuse Cerebral Microhemorrhage in a Patient with Sickle Cell Disease and Thalassemia
}

\author{
Smeer Salam, Resident (MD) ${ }^{1}$, Yasmeen Rauf, Resident $(M D)^{1}$, Deepak K Gulati, \\ Attending Physician (MD) ${ }^{1}$ \\ ${ }^{1}$ The Ohio State University Wexner Medical Center, Columbus, Ohio, USA
}

Introduction: Disseminated Intravascular Coagulation (DIC) is a consumptive coagulopathy affecting multiple organ systems that may frequently be observed in ICU patients secondary to several conditions, including trauma, sepsis, pancreatitis, blood transfusion, liver disease, pregnancy, surgery, and snake bites. It is characterized by acute activation of coagulation which results in thrombosis due to intravascular fibrin formation. Diffuse hemorrhages occur due to the consumption of platelets and coagulation factors.Neurologic complications of DIC include large vessel occlusion, subarachnoid hemorrhage, multiple cortical and brainstem hemorrhages, infarction and obtundation or coma. It very rarely presents and a cerebral disease.

Methods: We reviewed lab data, radiological and operative reports of a patient who developed with acute onset diffuse cerebral microhemorrhages after an orthopedic procedure.

Results: We present a case of a 51 year old female with sickle cell disease, B-thalassemia, protein $S$ deficiency, and deep venous thrombosis with pulmonary emboli who developed diffuse microhemorrhages in the cerebral cortex, white matter, cerebellum, and brainstem secondary to DIC immediately after arthroscopic subacromial decompression and repair of the right rotator cuff. Postoperatively patient was noted to have altered mental status, and an MRI with SWI sequence showed acute diffuse cerebral microhemorrhages. Her lab results were consistent with DIC likely secondary to sepsis from a pulmonary source. She was stabilized in the NCCU and received an IVC filter due to inability to be anticoagulated for concern of expansion of cerebral microhemorrhages.

Conclusions: DIC rarely presents with cerebral disease. It is associated with a higher mortality rate and requires prompt diagnosis and treatment. Our case report adds to very few cases of DIC presenting with cerebral disease. Prompt diagnosis and treatment are required for successful management.

Keywords: Cerebrovascular Disease, Hemorrhage, MRI, Coagulation, Imaging

Financial Disclosures: The authors had no disclosures.

Grant Support: None. 


\section{Poster 18}

\section{Continuous Intra-arterial Verapamil Effective Against Severe Reversible Cerebral Vasoconstriction Syndrome (RCVS)}

Pankaj Sharma, $M D^{1}$, Eytan Raz, $M D^{1}$, Maksim Shapiro, $M D^{1}$, Miguel Litao, $M D^{1}$, Omar Tanweer, $M D^{1}$, Peter Nelson, $M D^{1}$

'Department of Radiology, NYU school of Medicine, New York, New York, USA

Introduction: Reversible cerebral vasoconstriction syndrome (RCVS) is a transient disorder characterized by reversible constriction of the cerebral arteries.It is complicated by ischemic and hemorrhagic strokes with incidence as high as $54 \%$.

Methods: We present a case of 34 year old female with history of scleroderma, polymyositis overlap syndrome complicated by interstitial lung disease, systemic and pulmonary hypertension, who presented with chief complaints of worsening dyspnea, blurred vision and headache. Her BP was found to be 220 systolic with non focal neurological exam. MRI demonstrated multiple cortical infarcts in bilateral cerebral hemispheres. MRA revealed multifocal areas of stenosis in multiple intracranial arteries.

Results: She was started on oral verapamil for concerns of RCVS. On day 9, she became globally aphasic and plegic in right upper and lower extremities. Repeat MRI showed extension of infarct in multiple vascular territories. Cerebral angiogram showed multiple areas of severe arterial stenoses. Verapamil was injected into bilateral internal carotid arteries and left vertebral artery with a robust response in vessel caliber. On repeat evaluation after 24 hours benefit had subsided and in light of clinical decline decision was made to administer continuous infusion of verapamil in bilateral internal carotid arteries. The infusion was discontinued after 2 days with normal TCD results. MRI and MRA of brain on day13 of admission showed no new infarcts while demonstrating progressive clinical improvement.

Conclusions: Continuous intra-arterial verapamil infusion may have a role in treatment of refractory severe RCVS after less invasive options are exhausted.

Keywords: Ischemic And Hemorrhagic Stroke, Vasospasm, Vasospasm Intervention, Intra-Arterial Therapy

Financial Disclosures: The authors had no disclosures.

Grant Support: None. 
Poster 19

\section{Direct Use of a Distal Access Catheter Without Guiding Catheter for Intracranial Mechanical Thrombectomy}

Jenny P Tsai, MDCM, FRCPC ${ }^{1}$, Gabor Toth, $M D^{1}$

${ }^{1}$ Cleveland Clinic, Cleveland, Ohio, USA

Introduction: Establishing proximal support for cerebral mechanical thrombectomy may be limited by steno-occlusive disease and vascular tortuosity, if a guiding catheter cannot be safely maneuvered to the target cervical vessel. We report a case of successful mechanical thrombectomy with concomitant severe aortoiliac stenosis and tortuosity, where a 6 French (Fr) distal access catheter was utilized directly without a larger profile guiding catheter or shuttle sheath.

Methods: A 69-year-old male with a history of multiple aortic bifurcation stents presented with acute occlusion of the left internal carotid terminus. Initial vascular access demonstrated near-occlusion of the aortic bifurcation. After placement of a short 6 Fr sheath at the right groin, access across the stenosis was carefully obtained with a 0.035 " glidewire, with subsequent introduction of a standard 5 Fr diagnostic catheter to the cervical carotid. A 6 Fr SOFIA Plus distal access catheter (Microvention, Tustin, CA) was then exchanged for the diagnostic system.

Results: After uneventful crossing of the severe aortoiliac stenosis and tortuosity over an exchange wire, the intermediate catheter was effortlessly advanced to the carotid terminus occlusion site over a microwire and microcatheter without additional guiding catheter or shuttle sheath. Mechanical thrombectomy was successfully performed with TICI 3 reperfusion by retracting a stentriever directly into the SOFIA catheter under continuous aspiration. Time from puncture to first thrombectomy device deployment was 38 minutes. There were no complications.

Conclusions: Direct use of the SOFIA Plus distal access catheter without a guiding catheter or shuttle sheath provided a safe and stable access for mechanical thrombectomy. Potential limitations include the inability to use a balloon guide catheter for proximal flow control, and no option to perform stand-alone suction thrombectomy. This technique can be considered as a rescue therapy for patients with difficult vascular access due to severe aortoiliac disease and/or proximal vessel tortuosity.

Keywords: Access Catheters, Acute Ischemic Stroke Intervention, Endovascular Therapy, New Technique

Financial Disclosures: The authors had no disclosures.

Grant Support: None. 
Poster 20

\title{
Spontaneous Intracranial Artery Dissection and Acute Ischemic Stroke in Marfan Syndrome
}

\author{
Lili Velickovic Ostojic, $M D^{1}$, Johanna T Fifi, $M D^{2}$, Thomas J Oxley, $M D^{2}$, Maryna \\ Skliut, $M D^{1}$ \\ 'Department of Neurology, Mount Sinai Downtown, New York, New York, \\ USA; ${ }^{2}$ Department of Neurosurgery, Icahn School of Medicine at Mount Sinai, \\ New York, New York, USA
}

Introduction: Marfan syndrome is a rare connective tissue disorder caused by mutation of the fibrillin-1 gene, resulting in disruption of arterial elastic fibers and predisposing patients to aneurysm formation and arterial dissection. Neurovascular complications of Marfan syndrome are extremely rare with very few reported in literature. Only one pediatric case of intracranial vertebral artery dissection causing an ischemic stroke is reported. There are no prior reports on endovascular intervention in patients with cerebrovascular complications of Marfan syndrome. We present a case of acute infarct caused by spontaneous middle cerebral artery (MCA) dissection in an adult with Marfan syndrome in whom endovascular intervention was attempted.

Methods: A chart and imaging review of the case was performed, and literature search completed for arterial dissection and stroke in Marfan syndrome.

Results: A 34-year-old man with known Marfan syndrome presented to the emergency room with right MCA stroke. He was last known well 12 hours prior to presentation. NIHSS was 12 . Noncontrast head CT revealed a right temporal lobe hypodensity with sulcal effacement, consistent with early infarction in the right MCA territory. CT perfusion revealed perfusion mismatch in the affected area consistent with presence of a large penumbra. CTA showed occlusion of the right M1 segment. Digital subtraction angiography confirmed the occlusion and demonstrated irregularity of the right internal carotid artery starting just past the ophthalmic segment. The entire vessel was irregular, consistent with intra-arterial dissection. The microguidewire was attempted to be passed into the occluded branch. However, resistance was repeatedly encountered. The wire was thought to be entering the false lumen of the dissection. Due to the danger of further intervention, the decision was made to stop.

Conclusions: This is a unique case of intracranial dissection in an adult with Marfan syndrome. Endovascular intervention may be a treatment option in patients with neurovascular complications of Marfan syndrome.

Keywords: Ischemic Stroke, Acute Ischemic Stroke Intervention, Endovascular

Financial Disclosures: The authors had no disclosures.

Grant Support: None. 


\section{Poster 21}

\section{Acute Pipeline In-stent Thrombosis with Increased Vascular Enhancement Distal to the Pipeline Device}

Claribel D Wee, $M D^{1}$, Amar Swarnkar, $M D^{1}$

'SUNY Upstate Medical University, Syracuse, New York, USA

Introduction: 59 yo lady with the cerebrovascular risks of HLD, right MCA pipeline stenting on ASA $325 \mathrm{mg}$ daily who presents to the ED with left sided plegia, dysarthria, and aphasia. Notably, she had discontinued clopidogrel 1 week prior to presentation after 1 year of DAPT.

Methods: NIHSS 17 on arrival. CTA Head/Neck appeared to show a possible chronic left ICA occlusion as signal intensity distal to the flow diversion device on the right was increased in comparison. Immediately after the CTA $\mathrm{H} / \mathrm{N}$ was completed NIHSS decreased to 8 but remained consistent thereafter. Digital subtraction angiography revealed complete occlusion of the distal portion of the stent.

Results: Mechanical thrombectomy and intra-arterial thrombolysis were completed using a Penumbra device and augmented with Reopro. NIHSS at the time of discharge was 2 .

Conclusions: Flow diversion emerged as a viable endovascular therapy for treating intracranial aneurysms in 2011, has continued to gain popularity, and has grown in the scope of application to not only include wide-necked ICA aneurysms, but also unruptured- and posterior circulation aneurysms. Given their nascency, there are no guidelines regarding the duration of DAPT. However, DAPT is frequently started pre-operatively and typically continued for 3-6 months and followed by ASA alone thereafter. Further research should be conducted to further define factors which would better predict the need for lifelong DAPT as the thrombotic risk is intuitively increased with the large surface area of pipeline embolization devices. Additionally, the change of pressure gradient across the device may promote the formation of collaterals through an incompletely thrombosed aneurysm, as was the case in this presentation. This may result in perceived increased flow distal to an instent thrombosis and stroke physicians should be aware of this phenomenon as these devices continue be increasingly used.

Keywords: Acute Stroke, Aneurysm Embolization, Pipeline, Antiplatelet, Stenting

Financial Disclosures: The authors had no disclosures.

Grant Support: None. 


\section{Poster 22}

\section{Hemichorea-Hemiballism: An Unusual Presentation of Stroke in Takayasu Arteritis}

Cynthia B Zevallos, Dr ${ }^{1}$, Miguel A Quiñones, Dr' ${ }^{1}$, Marla L Gallo, Dr ${ }^{1}$, Max E Molina, Dr' ${ }^{1}$, Manuel A Moquillaza, Dr², Zaida L Rojas, Dr', Carlos Zapata, Dr ${ }^{1}$

${ }^{1}$ Hospital Nacional Guillermo Almenara Irigoyen, Lima, Peru; ${ }^{2}$ Oulton Institute. Neurorradiological Comprehensive Center, Córdoba, Argentina

Introduction: Takayasu arteritis (TA) is a large-vessel vasculitis affecting the aorta and its main branches. It occurs mainly in young women. It starts with general symptoms but 10 to $20 \%$ of patients develop neurological symptoms over the course of the disease, being rare as first signs, while $15.8 \%$ will develop a cerebrovascular disease (CVD).

Methods: Case Report

Results: We report the case of a 24-year-old woman patient with a history of TA dating back 10 years, with transient episodes of left hemichorea-hemiballism, presenting ipsilateral motor deficit and dysarthria two weeks later. The examination revealed blood pressure difference between both upper limbs and pulse asymmetry. The cerebral computed tomography (CT) showed subcortical hypodensity as well as hypodensity in right basal ganglia. The angiography revealed severe stenosis of right common carotid artery in its two proximal thirds and collateral filling in internal carotid. CT perfusion showed right frontoparietal subcortical hypoperfusion.

Conclusions: Hemodynamic mechanism is proposed as a stroke etiology in a patient with severe right extracranial carotid artery stenosis.

Keywords: Stroke, Extracranial Stenosis, Cerebral Blood Flow

Financial Disclosures: The authors had no disclosures.

Grant Support: None. 
Poster 23

\section{Safety and Efficacy of Large Bore Suction Catheters for Treatment of Cerebral Venous Sinus Thrombosis}

Sudeepta Dandapat, $M D^{1}$, Edgar Samaniego, $M D^{1}$, Ume Kiddy, $M D^{2}$, Fazeel Siddiqui, $M D^{2}$, Waldo Guerrero, $M D^{1}$, Nazan S Aksan, PhD' ${ }^{1}$, Binbin Zheng, MD', Colin Derdeyn, $M D^{1}$, Santiago Ortega Gutierrez, $M D^{1}$

${ }^{1}$ University of lowa, lowa City, lowa, USA; ${ }^{3}$ Southern Illinois University, Springfield, Illinois, USA

Introduction: We describe our experience using the new generation Penumbra large bore suction catheters for treatment of anticoagulation refractory CVST.

Methods: We collected data on 8 patients with CVST who received EAT under general anesthesia at two academic centers. Transfemoral arterial access was obtained in all patients, transfemoral venous access in 7 patients and 1 patient had transjugular access. Neuron Max (Penumbra, Alameda, CA) (6 patients) or the 6 french shuttle (Cook, Bloomington, IN) (2 patients) were used for venous access. The ACE 64 catheter (3/8) (Penumbra, Alameda, $\mathrm{CA})$ or the $5 \mathrm{MAX}$ catheter $(5 / 8)$ (Penumbra, Alameda, CA) were placed inside the guide as a large bore suction catheter and advanced to the affected sinus. Suction was performed using the Penumbra Pump (Penumbra, Alameda, CA). In 5 patients, 3MAX microcatheter (Penumbra, Alameda, CA) and in one patient Renegade microcatheter (Boston Scientific, Marlborough, MA) was introduced into the suction catheter to use as a clot separator.

Results: Seven patients had $>=2$ sinuses involved. One patient had associated $\mathrm{SAH}$, one cerebral edema, three venous infarctions and three intraparenchymal hemorrhage. Seven patients were treated with heparin prior to EAT and one patient with low molecular weight heparin. Reasons to pursue EAT in CVST patients were worsening mental status in 5 patients, status epilepticus in 2 patients and anticoagulation failure in 1 patient. The mean time from symptom onset to EAT was 4 days (range 1-14 days) with time from admission to EAT 2 days (range 0-4 days). Complete recanalization was obtained in 3 patients and partial in 5 patients. One patient required blood transfusion due to anemia on day after procedure. At discharge, there were 4 patients with mRS 0-2 and 2 patients with mRS 3.

Conclusions: Endovascular aspiration treatment using large bore suction catheters for CVST is a safe and effective approach for treatment of anticoagulation refractory CVST.

Keywords: Cerebral Sinus And Venous Thrombosis, Penumbra Max, New Technique

Financial Disclosures: The authors had no disclosures.

Grant Support: None. 


\section{Poster 24}

\section{Ultrasonography in Carotid Webs}

Diogo C Haussen, $M D^{1}$, Aaron Anderson, $M D^{1}$, Thomas Madaelil', Clara Barreira, $M D^{1}$, Song Kim, $M D^{1}$, Jonathan A Grossberg, $M D^{1}$, Michael Frankel, $M D^{1}$, Raul G Nogueira, $M D^{1}$

${ }^{1}$ Emory University School of Medicine/Grady Memorial Hospital, Atlanta, Georgia, USA

Introduction: Carotid web is a shelf-like linear filling defect in the posterior aspect of the internal carotid artery bulb. It is thought to represent an intimal variant of fibromuscular dysplasia and has been increasingly recognized as a cause of recurrent stroke. The performance of different imaging modalities for the diagnosis of carotid web is not known.

Methods: We retrospectively reviewed the ultrasound (US) results of cryptogenic stroke patients diagnosed with carotid web via CT angiography (CTA) in a comprehensive stroke center from 8/2014-5/2017. In patients that underwent carotid artery stenting for symptomatic carotid web, only the contralateral carotid was evaluated. Patients with CTA bulb thickness $\geq 3 \mathrm{~mm}$, indicative of atherosclerosis, were excluded.

Results: Of 20 patients with carotid web treated during the study period, 17 patients underwent US imaging (5 baseline and 12 after stenting), leading to 22 carotids for the analysis. Median age was 46.5[41.0-59.7]years, 76\% were female and $82 \%$ black. By CTA, 15/22 (68\%) had the presence of webs with a median web size of $2.8[2.4-3.5] \mathrm{mm}$. In the 15 CTAs with carotid webs, ultrasonography identified 9 "plaques" ( 6 mild and 3 moderate). Conversely, in seven carotids without webs (normal CTA), ultrasonography indicated mild plaques in two. None of the US reports characterized the lesions as webs. If considering plaques as indicative of webs, the US sensitivity was $60 \%$ and specificity $25 \%$. Out of the seven US reports that described plaque echogenicity in patients with webs, all reported hyperechogenicity. Median degree of stenosis (NASCET criteria) was higher by US as compared to CTA (30[10$30] \%$ vs $0[0-0] \%$; $\mathrm{p}<0.001)$.

Conclusions: Ultrasonography may have low sensitivity and specificity for the diagnosis of carotid webs. US findings are typically inconspicuous, associated with lesion hyperechogenicity, and with overestimated degree of stenosis.

Keywords: Stenting, Acute Stroke

Financial Disclosures: The authors had no disclosures.

Grant Support: None. 
Poster 25

\section{Carotid Angioplasty and Stenting Performed with MER ${ }^{\circledR}$ Carotid Stents in a Stroke Unit}

Adam Kobayashi, MD, Ph.D. ${ }^{1}$, Wojciech Czepiel, MD, Ph.D. ', Przemyslaw S Richter, $M D$, Ph.D. ${ }^{2}$, Marcin Kotkowski, $M D^{1}$

${ }^{1}$ Institute of Psychiatry and Neurology, Warsaw, Poland; ${ }^{2}$ Institute of Psychiatry and Neurology, Dr W. Roeffler Hospital, Warsaw, Pruszkow, Poland

Introduction: Carotid angioplasty and stenting (CAS) is one of the equivalent methods in stroke prevention for carotid artery stenosis. The MER ${ }^{\circ}$ carotid stent was marketed in 2013 and is the first carotid stent manufactured in Poland. This is the report of the safety and efficacy of CAS with the MER stent.

Methods: The study included 22 patients treated from 2014 to 2016 with the $\mathrm{MER}^{\circledast}$ stent in the setting of a stroke unit. Both symptomatic and asymptomatic stenosis were found eligible. Patients are being followed up after 6 months and annually for 5 years.

Results: 24 procedures were performer in 22 patients ( 2 had bilateral CAS). $66.7 \%$ were male, median age was 64 years (IQR $61-71$ ). $66.7 \%$ had right internal carotid artery stenosis. $4.2 \%$ had atrial fibrillation. $8.3 \%$ had restenosis ( 1 after endarterectomy, 1 after CAS), 2 patients had CAS done few days after thrombectomy for tandem occlusion. Implantation was successful in all patients, $91.7 \%$ had distal protection implanted during the procedure (In 2 patients protection device implantation was impossible due to a tight stenosis). $8,3 \%$ had groin hematoma, $4.2 \%$ had periprocedural bradycardia, $8,3 \%$ had hyperperfusional syndrome manifesting as transient unilateral headache. stwierdzono zespół hiperperfuzyjny pod postacią ustępującego połowiczego bólu głowy. None of the patients suffered stroke myocardial infarction, other vascular events nor died in the periprocedural stage nor on follow-up. The follow-up period ranged from 8 to 34 months. Restenosis rate was $0 \%, 1$ pacjent $(4,2 \%)$ had CABG during follow-up.

Conclusions: $\mathrm{MER}^{\oplus}$ carotid stents appear to be safe, efficacious, with good navigability and implantability in carotid stenosis. They also have high radial force. $\mathrm{MER}^{\oplus}$ carotid stents require further observation to fully assess their utility in clinical practice.

Keywords: Neuroprotection, Carotid Stenting And Angioplasty, Carotid Financial Disclosures: Principal Investigator for the MER clinical trial Grant Support: None. 


\title{
Poster 26
}

\section{Single-Center Experience in the Treatment of Acute Ischemic Stroke due to Tandem Occlusions}

\author{
Roberta Santos, $M D^{1}$, Manuel F Granja, $M D^{1}$, Romulo A Almeida, $M D^{1}$, Pedro \\ Aguilar-Salinas, $M D^{1}$, Jussie Lima, $M D^{2}$, Eric Sauvageau, $M D^{1}$, Ricardo A Hanel, \\ $M D, P h D^{1}$, Amin Aghaebrahim, MD ${ }^{1}$ \\ ${ }^{1}$ Baptist Neurological Institute, Jacksonville, Florida, USA; ${ }^{2}$ Department of \\ Neurology, University of Connecticut Health Center-Hartford Hospital, \\ Hartford, Connecticut, USA
}

Introduction: Acute ischemic stroke caused by tandem occlusions (TOs) present treatment challenges. Both proximal-to-distal and distal-to-proximal approaches have been reported without a clear consensus over which lesion should be treated first.

Methods: We conducted a single-center retrospective review of a prospectively maintained database of consecutive ischemic stroke patients from 2014 - 2017. The inclusion criteria consisted of patients undergoing endovascular treatment for TOs. Demographic, procedural, radiological, and clinical outcome data were analyzed. Good clinical outcome was defined as a mRS of $\leq 2$. An exploratory analysis was performed comparing interventional approaches (proximal-to-distal and distal-to-proximal).

Results: Twenty-three patients were identified for inclusion in this study. The average age of these patients was 61 years. Nine patients received IV tPA before undergoing endovascular treatment. Sixteen patients underwent a proximal-to-distal approach with first carotid artery stenting in 12 patients, angioplasty in 2 , and thrombectomy in 2 , followed by intracranial thrombectomy, whereas 7 patients underwent distal-to-proximal approach with intracranial thrombectomy performed first. For intracranial thrombectomy procedures, thrombectomy with stent-retrieval alone was used in 1 patient, aspiration alone was used in 1 , and a combination of aspiration and stentretriever thrombectomy was used in the remaining 21 patients. The average time to revascularization was 36 minutes $(n=16)$. Successful recanalization (TICI Grade 2b/3) was achieved in $22(96 \%)$ patients. Mean NIHSS scores were $18(\mathrm{p}<0.05)(\mathrm{n}=23)$ at presentation and $3(\mathrm{p}<0.05)(\mathrm{n}=23)$ at discharge. There was $1(0.04 \%)$ in-hospital death. In the exploratory analysis, we found no statistical difference between both groups. Favorable outcomes $(\mathrm{mRS} \leq 2)$ were achieved at 3 months in $8(67 \%)$ of 12 patients available for follow-up.

Conclusions: Simultaneous treatment of TOs is safe and feasible with favorable clinical outcomes at discharge and at 90 days. Further studies are required to determine which interventional approach is superior.

Keywords: Acute Ischemic Stroke Intervention, Carotid Stenting And Angioplasty, Mechanical Thrombectomy, Endovascular Therapy, TICI

Financial Disclosures: The authors had no disclosures.

Grant Support: None. 
Poster 27

\section{Benefit of Endovascular Therapy across Clinical Trial Design and Technique: A Single Center Pooled Analysis}

Tudor G Jovin, $M D^{1}$, Shashvat M Desai, $M D^{1}$, Ashutosh P Jadhav, MD PhD'

${ }^{1}$ University of Pittsburgh Medical Center, Pittsburgh, Pennsylvania, USA

Introduction: The practice of endovascular therapy has evolved dramatically over the last 10 years with randomized clinical trials investigating the benefit of thrombectomy in select patient populations based on imaging criteria and time of presentation as well as procedural technique. We sought to understand the benefit of thrombectomy in patients treated within the context of a clinical trial at a single academic center.

Methods: Data were analyzed from patients enrolled in randomized controlled trials investigating the benefit of intra-arterial therapy over medical therapy (IMSIII, ESCAPE, SWIFT PRIME and DAWN) between the years of 2007 and 2017 at a single academic referral center.

Results: A total of 130 patients (intervention group, $\mathrm{n}=80$; medical group, $\mathrm{n}=50$ ) were identified across four clinical trials (IMSIII, $\mathrm{n}=46$; ESCAPE, $\mathrm{n}$-24; SWIFT PRIME, $\mathrm{n}=14$; DAWN, $\mathrm{n}=46$ ). There were no significant differences between the treatment arm and control arm in terms of age, gender, baseline NIHSS and site of occlusion. There were no differences in rates of sICH, PH-2 or mortality in the intervention group versus medical group. Rates of good outcome were superior in the intervention group with early neurological recovery (defined as NIHSS of $0-1$ or drop in NIHSS of 8 points at 24 hours) at a higher rate of $53 \%$ vs $14 \%(\mathrm{p}=0.0001)$ and higher rates of functional independence ( 90 day mRS $0-2$ of $54 \%$ vs $26 \%, p=0.002$ ). In multivariate logistic regression analysis, lower baseline NIHSS and younger age were predictors of good outcome. There were comparable rates of good outcome irrespective of clinical trial, imaging selection criteria (CTP vs MRI), early versus late time window design (0-6 hours versus 6-24 hours) and procedural technique (Merci/EKOS vs Solitaire/Trevo).

Conclusions: At a large academic center, the benefit of endovascular therapy over medical therapy is observed irrespective of clinical trial design, patient selection or procedural technique.

Keywords: Acute Stroke, Acute Ischemic Stroke Intervention, Clinical Trial, Endovascular Therapy, Clinical Investigations

Financial Disclosures: Consultant: Ownership Interest: Silk Road-modest, Anaconda-modest, Consultant: Ownership Interest: Blockade Medmodest Consultant: Covidien/Medtronic: unpaid Consultant: Strykerun paidPI: REVASCAT, unpaid PI: DAWN(Stryker), unpaid Consultant: NeuraviConsultant(DSMB): Codman

Grant Support: None. 


\section{Poster 28}

\section{Challenges in the Endovascular Management of Basilar Artery Occlusion: Case Report and Review of the Literature}

Zachary Bulwa, M.D. ${ }^{1}$

${ }^{1}$ The University of Chicago, Chicago, Illinois, USA

Introduction: Due to the devastating nature and high mortality rate associated with basilar artery occlusion, vascular neurologists and neurointerventionalists have been eager to improve outcomes. In older studies intra-arterial and intravenous thrombolytic therapy initially showed poor results, but newer-era mechanical thrombectomy devices have offered promise. It has been shown that early recanalization is related to improved outcomes, although successful and sustained recanalization can be challenging in the posterior circulation. Limited evidence has demonstrated that recanalization rates may be related to characteristics of the thrombus and chronic atherosclerosis. Clinical and radiographic presentations can assist in predicting clinical outcome regardless of recanalization and may be used to avoid unnecessary intervention in futile cases. Here we discuss a very timely case of a 73-year-old female who presented with stuttering dizziness and new onset left hemibody weakness found to have acute basilar artery occlusion. In describing the challenges of her acute management we will review current interventions for basilar artery occlusion and adjunctive medical and endovascular therapy used to improve outcome.

Methods: -

Results: -

Conclusions: -

Keywords: Acute Ischemic Stroke Intervention, Basilar, Endovascular Therapy

Financial Disclosures: The authors had no disclosures.

Grant Support: None. 
Poster 29

\title{
EpxEpilepsy: A SMS-Based Intervention for Patients with Epilepsy
}

\author{
Rajat K Duggirala, Bachelors of Science ${ }^{1}$, Robert Peters, Bachelor of Arts ${ }^{2}$, \\ Stephanie Zhang, Bachelors of Science ${ }^{1}$ \\ ${ }^{1}$ Saint Louis University School of Medicine, St. Louis, Missouri, USA; \\ ${ }^{2}$ Washington University in St. Louis School of Medicine, St. Louis, Missouri, \\ USA
}

Introduction: By utilizing a telecommunication tool, EpxEpilepsy, our goal was to improve management of seizures in epileptic patients. Daily short message service (SMS) and/or phone calls allowed us to observe if this interactive platform could better track an epileptic patient's seizures, manage medication, and assist in bettering communication between patients and their providers. Current self-recorded seizure frequency logs can be imprecise, further compromising their quality of care. Offering prompt interventions are crucial in observing a median reduction in seizure frequency.

Methods: EpxEpilepsy is an automated service that prompts patients to track their seizure frequency which is summarized and reported to the provider on a weekly basis. Since providers can set the baseline number of seizures for each patient, EpxEpilepsy will identify patients who have a seizure frequency outside the threshold parameters. Through the Washington University Specialty Care Clinic, 10 patients were enrolled, receiving one text message for 24 weeks. The goal was to observe how the intervention could help patients track and reduce seizures and assist physicians in better monitoring and communicating with patients.

Results: We were able to capture 31 triggers where patients experienced an above-baseline amount of seizures, and facilitated 13 phone calls totaling approximately 67 minutes between physicians and patients. On average, patients were 2 times as likely to respond to the intervention when they have seizure activity greater than their threshold during the first 7 weeks of enrollment.

Conclusions: Patients are mostly utilizing this system and responding to the intervention when they experience an above normal amount of seizures. The relatively high response rate triggering alerts shows that there is potential for this intervention to help patients in tracking and communicating their seizures to their physicians. Moving forward, a randomized control trial with a larger sample size can offer greater insight into the usability of this intervention.

Keywords: Care, Clinical Investigations

Financial Disclosures: The authors had no disclosures.

Grant Support: None. 
DTI Findings of Corticospinal-Tract Recovery After an IntraArterial Mesenchymal-Stem-Cell Infusion in a Canine Stroke Model

Luis Guada, $M D^{1}$, Pradip Pattany, PhD'2, Kevin Ramdas, $M D^{2}$, Kunakorn

Atchaneeyasakul, $M D^{2}$, Dileep Yavagal, $M D^{2}$

${ }^{1}$ University of Miami Miller School of Medicine, Miami, Florida, USA;

${ }^{2}$ University of Miami, Miami, Florida, USA

Introduction: The purpose of this study was to compare DTI biomarkers of neurological recovery in a canine stroke model over one month after ipsilateral intra-arterial (IA) Mesenchymal Stem Cell (MSC) infusion vs control.

Methods: Mongrel-Hounds $(n=9)$, aged 12-36-months. MCA-stroke was induced via endovascular using a detachable-coil over 35-80 min. MSC (1-40 million/240 cc) or saline/240 cc were infused IA, 48 hrs post-stroke. Brain-MRIs were performed prior to IA infusion, and $15 \& 30$ days poststroke. FA values of the ipsilesional CST were determined. DTT of the CST was generated. Weekly neurological score-evaluations were performed. Comparison of FA-DTT at 30 days post-stroke between treated vs control.

Results: We observed improvement in FA-values 30 days post-stroke in the MSC-group $(0.085 \pm 0.025, \mathrm{n}=5$ vs $0.068 \pm 0.058, \mathrm{n}=4: \mathrm{p}=0.82)$ and $\mathrm{a}$ qualitative increase in CST-caliber, not seen in controls. FA-increases in treated animals correlating with faster motor-recovery. FA did not change in the control group.

Conclusions: DTI-DTT imaging post IA-MSC therapy showed an increased FA and caliber of CST (DTT) correlating with motor recovery. These findings could support further development of DTI-DTT biomarkers to measure neurological-recovery in experimental models as well in clinical trials of novel stroke-therapies.

Keywords: Intra-Arterial Therapy, Stem Cell Therapy, MRI, New Innovation, Stroke

Financial Disclosures: The authors had no disclosures.

Grant Support: None. 
Poster 31

\title{
Safety and Clinical Outcomes after Transverse Venous Sinus Stenting for Treatment of Idiopathic Intracranial Hypertension: Single Center Experience
}

\author{
Ashish Kulhari, M.D. ', Siddhart Mehta' ${ }^{1}$ Rebekah Amarini', Spozhmy Panezai', \\ Jawad Kirmani, $M D^{\prime}$ \\ 1JFK Stroke \& Neurovascular Center, Seton Hall Hackensack Meridian School \\ of Medicine, Edison, New Jersey, USA
}

Introduction: Idiopathic intracranial hypertension (IIH) is a disorder characterized by signs and symptoms of increased intracranial pressure without structural cause seen on conventional imaging. Unilateral or bilateral transverse sinus or transverse-sigmoid junction stenosis is a very common finding in these patients. There is ongoing debate whether venous sinus stenosis is the cause of IIH or result of it. Multiple case reports and case series have proven venous sinus stenting (VSS) to be very effective in medically refractory IIH. Through this study, we want to share our experience with venous stenting in patients with IIH who had transverse sinus or transversesigmoid sinus junction stenosis.

Methods: All patient with medically refractory IIH who underwent venous sinus stenting (VSS) at our university affiliate community comprehensive stroke center in 2017 were analyzed.

Results: Our neuroophthalmology services identified four patients that had medically refractory IIH and underwent VSS or angioplasty. Mean age was 47 . Seventy five percent of patients were women $(n=3)$. Headache was the most common symptom (100\%) followed by transient visual obscurations $(75 \%, \mathrm{n}=3)$ and pulsatile tinnitus $(25 \% ; \mathrm{n}=1)$. All patients were found to have bilateral papilledema. Mean lumbar opening pressure was 36. All patients were on maximum doses of acetazolamide and diuretics. Half of the patients had right transverse sinus stenosis with hypoplastic left transverse sinus stenosis $(\mathrm{n}=2)$ and other half had bilateral transverse sinus stenosis $(\mathrm{n}=2)$. Mean pressure gradient across the transverse sinus was $17(32 \pm 15)$. Three patients were treated with transverse sinus stenting and one with angioplasty. All patients were able to come off their medications with significant improvement in symptoms and visual fields. No complications occurred during intervention.

Conclusions: Transverse sinus angioplasty $+/$ - stenting is a safe and effective means of treating IIH. Larger studies are required to support our results. *Due to insufficient provided space for supporting authors, we had put all authors in Title*

Keywords: Intracranial Stenosis Stenting And Angioplasty

Financial Disclosures: The authors had no disclosures.

Grant Support: None. 
Poster 32

Technical Description of Cerebrovenous Stenting: A Study of Operator Preference for Patients with Refractory Intracranial Hypertension

\author{
Ashish Kulhari, M.D. ${ }^{1}$, Siddhart Mehta' ${ }^{1}$, Rebekah Amarini', Spozhmy Panezai', \\ Jawad Kirmani ${ }^{1}$ \\ 1JFK Stroke \& Neurovascular Center, Seton Hall Hackensack Meridian School \\ of Medicine, Edison, New Jersey, USA
}

Introduction: Venous sinus stenting (VSS) has been gaining popularity for the treatment of refractory Idiopathic intracranial hypertension (IIH). Multiple case reports and series have described different techniques for venous stenting depending on challenges they face. After compiling the overview of techniques and our experience we describe the best sequence of technique in this case series.

Methods: All patient with medically refractory IIH who underwent VSS at our university affiliated community comprehensive stroke center in 2017 were analyzed. Angiogram images and clinical charts were reviewed.

Results: Femoral Arterial (5F cordis sheath, Left Femoral Access) and venous access ( $8 \mathrm{~F} 30 \mathrm{~cm}$ cook sheath) is gained at the start of the case and a diagnostic angiogram was obtained via $4 \mathrm{~F}$ Vert diagnostic catheter placed in RICA. A 8-French guide catheter (Infinity, Stryker) was advanced into the right jugular bulb. A 4-French $150 \mathrm{~cm}$ Bernstein hydrophilic glide catheter was used over the 035 guide wire (Terumo Inc.) to transverse and navigate through the stenotic right transverse sinus, torcula and into superior saggital sinus (SSS). The guide wire was removed and a standard arterial pressure transducer was calibrated and connected to the Bernstein catheter. Venous manometry was then performed, with recording of mean venous pressures in the SSS, torcula, pre- and post- transverse sinus (TS), sigmoid sinus (SS), and IJV. A difference of $\geq 10 \mathrm{~mm} \mathrm{Hg}$ across the stenosed segment was required to proceed with stenting. Bernstein catheter was again navigated to SSS over the 035 glide wire. It was then exchanged with 035 extra stiff Amplatzer wire. A 10X40 mm Protégé self expanding stent was then inserted over the Amplatzer wire and deployed in the transverse sinus. No angioplasty is necessary. Post stenting arterial run showed no residual stenosis with improved flow through the stent. Post stenting venous manometry confirmed equal mean pressures across the stent.

Conclusions: A rapid and reliable technique of this emerging neurointerventional procedure indication has been described here. Future specialized devices for cerebrovenous system may obviate the necessity of improvising with devices primarily designed for other indications. ${ }^{*}$ Due to insufficient space for supporting authors, we included authors in Title *

Keywords: Intracranial Stenosis Stenting And Angioplasty

Financial Disclosures: The authors had no disclosures.

Grant Support: None. 
Poster 33

\title{
A Case of Refractory Reversible Cerebral Vasoconstriction Syndrome Managed with Intra-Arterial Nicardipine
}

\author{
Stanislav Naydin, $M D^{1}$, Hana Choe, $M D^{2}$, Osman S Kozak, $M D^{2}$, Qaisar A Shah, \\ $M D^{2}$, Larami G MacKenzie, $M D^{2}$ \\ 'Department of Neurology - Drexel University College of Medicine, \\ Philadelphia, Pennsylvania, USA; ${ }^{2}$ Neurointerventional - Abington Jefferson \\ Health, Abington, Pennsylvania, USA
}

Introduction: Reversible Cerebral Vasoconstriction Syndrome (RCVS) is a neurological diagnosis that presents with sudden thunderclap headaches and features intracranial vascular focal constrictions.[1] RCVS can lead to dire consequences including permanent disability and death. To date there is no established guideline for diagnosis or treatment.

Methods: The response to intra-arterial (IA) calcium channel blockers for treatment has been demonstrated. Vasoconstriction responding to IA vasodilators may even be a diagnostic feature of RCVS.[2]

Results: We present a case of severe RCVS managed via serial administration of IA nicardipine. 54 year old female with history of tobacco abuse, hypertension, hyperlipidemia, migraines, recent angioplasty and stent intervention for left subclavian stenosis, posterior circulation transient ischemic attacks, and limb claudication who presented with severe headaches and confusion. MRI showed left parietal lobe restricted diffusion. CTA demonstrated (known) 76\% stenosis of left ICA with narrowing versus partially occlusive embolus of M1 and M2 segments of the left MCA, consistent with atherosclerosis. Left ICA stenosis was considered symptomatic, and stent was placed. After which left hemispheric function improved. During stenting, angiogram demonstrated multifocal areas of constriction. Vasculitis workup including lumbar puncture was negative. Several days later the patient developed left sided weakness. CTA and then conventional angiograms were obtained, showing new stenoses of multiple right hemispheric branches, consistent with RCVS. Patient was placed on a calcium channel blocker.[3] Symptoms did not remit. Conventional angiogram was performed, with arterial relaxation at stenotic sites following IA nicardipine. The patient showed clinical improvement following treatment, however focal neurologic symptoms would recur, leading to repeated angiograms for IA vasodilator therapy over 5 days. Patient eventually remained stable and was able to be discharged.

Conclusions: This case demonstrates that in the setting of RCVS with severe neurologic deficits and inadequate response to medical therapy, repeated IA nicardipine may contribute to favorable outcome.

Keywords: Ischemic Stroke, Cns Vasculitis, Endovascular Therapy, TIA, Intra-Arterial Therapy

Financial Disclosures: The authors had no disclosures.

Grant Support: None. 


\section{Poster 34}

\section{Intermittent Intra-Arterial Saline Flushing After Stroke Revascularization in Rats}

Prad Selvan, $M D^{1}$, Frank C Barone, $P h D^{2}$

${ }^{1}$ University of California San Diego, San Diego, California, USA; ${ }^{2}$ SUNY

Downstate, Brooklyn, New York, USA

Introduction: Mechanical thrombectomy (MT) for large vessel occlusive (LVO) stroke provides an opportunity to deliver targeted intra-arterial (IA) adjunctive therapies (ATs) to the ischemic brain. We developed a model of selective IA catheterization after transient middle cerebral artery occlusion (tMCAO) in rats to investigate targeted ATs. Here we report the brain protective effects of targeted IA flushing with normal saline as an AT to LVO revascularization.

Methods: We incorporated a continuous internal carotid artery (ICA) catheter infusion system after tMCAO in Sprague Dawley rats (300-350 g). Two groups were studied ( $\mathrm{N}=6$ /group): Control (tMCAO with IA catheter only) and Flushing (tMCAO with IA catheter and brief intermittent flushing). The Flushing group received $1 \mathrm{~min}$ infusions of normal saline at $2 \mathrm{cc} /$ min immediately, $1 \mathrm{hr}$, and $2 \mathrm{hrs}$ after reperfusion. Laser Speckle Imaging was used to measure microvascular perfusion. Functional measurements and histological analysis were performed at $24 \mathrm{hrs}$ post-reperfusion.

Results: Incorporating a IA catheter system after tMCAO was safe in rats. Flushing saline into the ICA caused a targeted, significantly increased microvascular perfusion of the ischemic MCA territory $(\mathrm{P}<0.05)$. Histological analysis demonstrated a significant $(\mathrm{P}<0.05)$ decrease in total and cortical infarct size and cortical and striatal swelling. Functional measurements were also significantly decreased $(\mathrm{P}<0.05)$ by intermittent flushing.

Conclusions: Continuous intra-arterial catheter access can be safely incorporated into a rat model of transient middle cerebral artery occlusion. Here we demonstrate that intermittent targeted flushing of the ischemic brain can be effective as an AT to LVO revascularization. It is now possible and necessary to investigate targeted IA therapeutics after tMCAO in rats.

Keywords: Neuroprotection

Financial Disclosures: Research support from Society of Vascular and Interventional Neurology

Grant Support: None. 
Poster 35

\section{Expanding Time Windows Present Niche Endovascular Stroke Care Opportunities}

Faheem G Sheriff, $M D^{1}$, Joshua A Hirsch, $M D^{2}$, Christopher J Stapleton, $M D^{2}$, Matthew Koch, $M D^{2}$, Susan C Williams, $M D^{2}$, James Rabinov, $M D^{2}$, Aman B Patel, $M D^{2}$, Thabele Leslie-Mazwi, $M D^{1}$

${ }^{1}$ Massachussets General Hospital; Harvard Medical School, Boston, Massachusetts, USA; ${ }^{2}$ Massachusetts General Hospital, Boston, Massachusetts, USA

Introduction: The DAWN and DEFUSE 3 trials have major implications for strokes caused by large vessel occlusion (LVO) that present outside of conventional treatment windows. We present our post-DAWN experience with a unique subpopulation, patients undergoing cardiothoracic surgery.

Methods: A prospective institutional stroke database was reviewed between May and August 2017 for cardiothoracic patients with LVO.

Results: Five cardiothoracic patients met criteria for emergent thrombectomy due to LVO. All were males with a range of cardiac pathology: all had left heart surgical endocardial violation. At presentation, median NIHSS was 16 and ASPECTS ranged from 7 to 9. All patients had M1 occlusions. Patients fell into two categories: detection of new deficit in the alert patient 1-2 days after surgery (Last seen well (LSW) to diagnosis median of 120 minutes) or detection of deficit on emergence of the sedated patient from anesthesia (LSW to diagnosis median of 750 minutes). The latter were patients placed on cardiopulmonary bypass, and warmed before being transferred to cardiac critical care for weaning of sedation. Median procedure duration was $56 \mathrm{~min}$ utes. Only $2 / 5$ patients achieved mTICI scores of $\geq 2 \mathrm{~b}$. Clot pathology was obtained in $2 / 5$ cases and showed myocardium in one case (mTICI $2 b$ ) and fat in another (mTICI 1). Hemorrhagic conversion occurred in $3 / 5$ patients (one PH2). NIHSS at day seven ranged from 0-21 (median 7).

Conclusions: DAWN and DEFUSE 3 open therapy to patients previously outside all treatment windows. Patients with deficit on emergence from anesthesia fall into this category. Endovascular stroke therapy for patients post cardiothoracic surgery is characterized by late treatment windows, and technically challenging procedures (unusual emboli pathology translate into lower recanalization rates, higher complication rates, and longer procedure durations). However, these patients represent a niche where late window treatment may positively modify the complication profile of cardiothoracic surgery.

Keywords: Acute Stroke, Treatment, Revascularization, Endovascular Therapy

Financial Disclosures: The authors had no disclosures.

Grant Support: None. 
Poster 36

\section{Traumatic Vertebral and Carotid Artery Dissections Related to Motor Vehicle Accidents: Injury Characteristics and Outcomes}

Rakesh Khatri, $M D^{1}$, Mohtashim Arbaab Qureshi, $M D^{1}$, Mohammad Rauf Afzal, $M D^{\prime}$, Anantha Ramana Vellipuram, $M D^{1}$, Salvador Cruz-Flores, $M D^{\prime}$, Gustavo J Rodriguez, $M D^{1}$, Alberto Maud, $M D^{1}$

${ }^{1}$ Texas Tech University Health Sciences Center, El Paso, Texas, USA

Introduction: Comparative analysis between carotid and vertebral artery dissections related to motor vehicle trauma at the national level is not well studied. Our objective was to compare patterns of injury and in-hospital outcomes in motor vehicle related traumatic carotid versus vertebral artery dissections at national level.

Methods: A retrospective study was conducted using a national database (years 20011 to 2014).To identify the patients from database who suffered motor vehicle, we used ICD-9-CM, E- codes. Out of this motor vehicle accident traumatic group, we identified patients admitted with primary diagnosis of carotid and vertebral artery dissection using respective codes: 443.21 and 443.24 .

Results: A total of 2969 patients had either carotid $(n=1300)$ or vertebral $(n=1669)$ artery dissection. Vertebral artery dissection patients were more commonly associated with (83\%) fractures of cervical vertebrae either without spinal cord injury (66.5\%) or with spinal cord injury (16.5\%). There was no statistically significant difference in the rate of ischemic stroke $(22 \%$ carotid vs $16.6 \%$ vertebral, $\mathrm{p}=0.10)$. Carotid dissection patients had more traumatic subarachnoid hemorrhage $(17.8 \%$ vs $11 \%, \mathrm{p}=0.02)$, traumatic brain injury $(68.8 \%$ vs $40.6 \% \mathrm{p}<.01)$ and other organs injuries. They also had increased length of stay ( 15.5 days vs 10.2 days, $\mathrm{p}=0.01)$ and higher moderate to severe disability $(61.8 \%$ vs $46.1 \%, \mathrm{p}=0.02)$ and the difference remained the same after adjusting for age, gender and the presence of other injuries (OR 1.679, CI $1.136-2.483, \mathrm{p}=0.01$ ). There was no difference in mortality $(12.0 \%$ carotid artery dissection vs $7.8 \%$ vertebral artery dissection, $\mathrm{p}=$ $0.110)$.

Conclusions: Carotid artery dissection related to motor vehicle accidents are associated with other multiple organ injuries and bone fractures suggesting higher impact trauma, and they lead to higher moderate to severe disability compared to vertebral artery dissections. There is an association of vertebral artery dissection and the presence of vertebrae fractures

Keywords: Carotid, Vertebral, Ruptured

Financial Disclosures: The authors had no disclosures.

Grant Support: None. 
Poster 37

\section{Vertebral Artery Congenital Variant in Velocardiofacial Syndrome (DiGeorge Syndrome)}

Mohamed Shehabeldin, MD ${ }^{1}$, Yazan J Alderazi, MD ${ }^{1}$

${ }^{1}$ Texas Tech University Health Sciences Center, Lubbock, Texas, USA

Introduction: Velocardiofacial syndrome (DiGeorge syndrome); is not an uncommon syndrome, caused by deletion of 22q11.2 chromosome which occurs in 1:4000 births. It is commonly associated with vascular anomalies in the cardiac and pulmonary vasculature. Also, anomalies in the carotid and vertebral arteries have been reported in literature.

Methods: Retrospective patient chart review and review of imaging in a patient with variant vertebral artery origin and course.

Results: A 26-year-old woman with history of velocardiofacial syndrome who underwent multiple open-heart surgeries during her childhood. She was referred to our vascular neurology clinic for evaluate of abnormal vessels found in MRA that was performed for evaluate of depressive and behavioral symptoms. CTA head and neck was performed for further assessment. MRA and CTA head and neck studies showed an interesting congenital variant of her vertebral artery anatomy. The distal cervical left internal carotid artery gave rise to a left dominant vertebral artery at the level of C1-2, which continued as the intracranial segment of the left vertebral artery. The dominant anastomotic channel represents a dominant $\mathrm{C} 1$ segmental artery. The proximal segments of the left vertebral artery were hypoplastic and joined the aberrant but dominant left vertebral artery at the $\mathrm{V} 3$ level. The right vertebral artery is hypoplastic and arises normally from the right subclavian artery and had a normal course.

Conclusions: We demonstrate a case of variant vertebral artery supplied via the internal carotid artery via the dominant $\mathrm{C} 1$ segmental artery anastomosis in association with velocardiofacial syndrome. This expands the spectrum of vascular anomalies of the velocardiofacial syndrome. Unfamiliarity with this condition might be very challenging during endovascular and surgical management of different cerebrovascular and cervical spine conditions.

Keywords: Vertebral, Vascular Imaging, Carotid

Financial Disclosures: The authors had no disclosures.

Grant Support: None. 


\section{Poster 38}

\section{Selection of Candidates for Delayed Recanalization with Endovascular Therapy After Acute Ischemic Stroke}

Oh Young Bang, $M D^{1}$, Jong-Won Chung, $M D^{1}$, Keon Ha Kim, $M D^{1}$, Pyoung Jeon, $M D^{1}$, Woo-Keun Seo, $M D^{1}$, Gyeong-Moon Kim, $M D^{1}$

'Samsung Medical Center, Seoul, Korea, Republic of

Introduction: Although the beneficial effect of endovascular therapy (EVT) was confirmed in acute ischemic stroke (AIS), not all patients showed favorable outcome after EVT. There have been efforts to select candidate patients for EVT using triage methods, including target mismatch profile (TMP), clinical-core mismatch (CCM), and collateral circulations. We compared these 3 triage methods in terms of the number of patients eligible for EVT, and 90th day clinical outcome and day 7 infarct growth after delayed ( $>5 \mathrm{~h}$ after onset) recanalization.

Methods: We included patients who presented within $8 \mathrm{~h}$ of onset; who underwent pretreatment brain MRI; who showed a NIHSS score of 4 or more and ICA and/or proximal MCA occlusion; and who received EVT. Collateral grading was assessed using collateral flow map generated based on contrastMR perfusion source data. CCM was defined with the DAWN trial methods, and TMP was classified based on the DEFUSE-3 trial definition.

Results: A total of 109 patients were included in this study. Number of patients eligible for EVT was varied greatly among 3 triage methods. Only 46 (42.2\%) of patients were eligible for EVT by all triage methods. The number of eligible patients were higher in TMP (97 patients, 89.0\%) selection than in collaterals (73 patients, 67.0\%) or CCM (75 patients, 68.8\%) selection. Patients who were ineligible for EVT by one criteria often could be eligible by other criteria. Among patients who had successful recanalization after $5 \mathrm{~h}$ of onset, good outcome was best predicted by collateral parameter than by CCM or TMP, whereas the degree of infarct growth was larger in patients with poor collaterals or absence of TMP.

Conclusions: The triage methods may provide differential information each other. In the studied cohort, collateral-based triage could best predict favorable outcome and infarct growth after delayed recanalization. Further studies are needed in a larger prospective cohort.

Keywords: Acute Stroke, Endovascular Therapy, MRI

Financial Disclosures: The authors had no disclosures.

Grant Support: None. 
Poster 39

\title{
Comparing Safety and Efficacy of Biplane versus Monoplane Angiography in Hyperacute Neuroendovascular Therapy
}

\author{
Gregory D Kurgansky, $B A^{1}$, Phillip Ye, $B S^{1}$, Ryan $T B o, M D^{1}$, Jeremy $M$ Liff, $M D^{1}$, \\ Karthikeyan Arcot, $M D^{1}$, David Turkel-Parrella, $M D^{1}$, Jeffrey Farkas, $M D^{1}$, Ambooj \\ Tiwari, $M D M P H^{2}$
}

${ }^{1}$ NYU Langone Hospital - Brooklyn Interventional Neuro Associates, Brooklyn, New York, USA; ${ }^{2}$ NYU Langone Hospital - Brooklyn, Brooklyn, New York, USA

Introduction: Most stroke/neuro-interventional centers require advanced biplane imaging for evaluation of complex cerebrovascular lesions. Purchasing such equipment is cost-prohibitive for many hospital systems. Additionally, operator-preference often prevents the use of other imaging platforms as a back up for acute neuro-interventional cases. However, most hospitals are often equipped with multiple single plane imaging platforms for IR \& Cardiac purposes. Advanced single plane imaging in most catheterization labs provide reasonable penetration and field of view (FOV) for doing acute cases like mechanical thrombectomy.

Methods: A retrospective review of our multi-center database of acute stroke patients treated with endovascular therapy was performed. 207 patients were categorized by type of imaging platform on which thrombectomy was performed and relevant angiographic and clinical data was gathered. Primary outcome was measured using angiographic outcome. This included comparisons between two groups of TICI scores: TICI $0-2 \mathrm{~A}$ vs. TICI $2 \mathrm{~B}-3$ and TICI $2 \mathrm{~B}$ vs. TICI $2 \mathrm{C} / 3$. Secondary outcome was safety which was reported as incidence of intracranial hemorrhage between the two groups

Results: 146 biplane patients achieved scores of $2 \mathrm{~B}$ or higher, meanwhile 12 biplane patients received scores of $2 \mathrm{~A}$ or lower. 44 of 49 single plane patients achieved TICI scores of 2B-3 while 5 had scores of $0-2 \mathrm{~A}$, showing no significant difference ( $\mathrm{p}>0.05)$. In a second comparison, 97 biplane patients that had outcomes of $2 \mathrm{C}$ or 3 , and 49 patients with outcomes of $2 \mathrm{~B}$; while 29 monoplane patients achieved a score of $2 c$ or 3 and 15 with $2 B(p>0.05)$. For our secondary measure, ICH in the biplane group was 38/146 and in monoplane group was $9 / 49$, the difference not being statistically significant ( $\mathrm{p}>$ 0.05)

Conclusions: There was no significant difference in safety or efficacy outcomes when comparing thrombectomies performed using biplane vs. monoplane imaging. Thrombectomies performed with either imaging system is equally safe and effective.

Keywords: Acute Ischemic Stroke Intervention, Angiographic Technology, TICI, Vascular Imaging, Mechanical Thrombectomy

Financial Disclosures: The authors had no disclosures.

Grant Support: None. 
Poster 40

Abstract withdrawn 


\title{
Poster 41
}

\section{Flat- Panel Cone Beam Computed Tomography is a NOT a Reliable Predictor for Early Changes in Ischemic Stroke Patients with Large Vessel Occlusions (LVO)}

\author{
Amrinder Singh ${ }^{1}$, Ashish Kulhari', Siddhart Mehta', Briana DeCarvalho', Hemal \\ Patel', Spozhmy Panezai', Jawad Kirmani, $M D^{1}$ \\ 1 JFK Stroke and Neurovascular Center, Seton Hall Hackensack Meridian School \\ of Medicine, Edison, New Jersey, USA
}

Introduction: The Alberta stroke program early CT score (ASPECTS) is a standardized tool to quantify the core infarct in MCA territory stroke. Current practice involves transporting a patient for CT imaging followed by transport of patients to the angiography suite for acute Neurointervention. In order to expedite this process and potentially improve patient outcomes, benefit may be derived from performing imaging directly in the angio suite. C-arm Cone Beam CT (CBCT) is an emerging technology being integrated into many of the leading angiography systems in neurointerventional suites. This system incorporates 3D functionality and extends the capability of $\mathrm{C}$-arm imaging to include soft-tissue applications by facilitating the detection of low-contrast objects.

Methods: Between 2011-2016, CBCT was performed in 8 patients during neurointervention procedures at our university affiliated community based comprehensive stroke center, for early detection of Intracerebral Haemorrhage, due to suspicion of peri-procedural perforation complication.

Results: Of a total 221 stroke patient that received acute stroke intervention, a total of 8 patients received CBCT who underwent neurointervention. Six of 8 patients (75\%) patients presented with acute ischemic stroke, 1 with cerebral aneurysm and 1 with SAH. Mean age was 73 years (Range 49-93); $62.5 \%(5 / 8)$ were females. With the use of CBCT intra-operatively, Left basal ganglia hemorrhage, IVH with midline shift was detected in patient who underwent L ICA stenting and Left M1 mechanical thrombectomy. SAH patient's CBCT revealed worsening diffuse SAH with hydrocephalus. All patients had thin sliced CT head prior and after procedure for comparison of ICH with CBCT. These findings were confirmed on Thin sliced CT. No ICH/ IVH was noticed on rest of the patients. Volume of ICH was not calculated on CBCT.

Conclusions: We concluded that the CBCT could provide useful information for early infarcts in M1-M6 areas of MCA LVO's. Early deep cortical infarcts can be easily missed on CBCT. Limitation of this study was low patient population. A large population based prospective study is needed to analyze future use of CBCT to expedite decision making in patients with LVO. *Due to insufficient provided space for supporting authors, we had to put all authors in Title*

Keywords: Acute Stroke, Angiographic Ct

Financial Disclosures: The authors had no disclosures.

Grant Support: None. 
Poster 42

\section{Impact of Ultra-Rapid-Sequential IV/IA Contrast on Incidence of CIN in a Comprehensive Stroke Center}

Phillip Ye, $B A^{1}$, Gregory Kurgansky, $B A^{1}$, Jeremy Liff, $M D^{1}$, Jeffrey Farkas, $M D^{1}$, Karthikeyan Arcot, $M D^{1}$, David Turkel-Parrella, $M D^{1}$, Ambooj Tiwari, $M D D^{2}{ }^{1} H^{1}$, Jennifer A Frontera, $M D^{2}$

${ }^{1} \mathrm{NYU}$ Langone Hospital - Brooklyn Interventional Neuro Associates, Brooklyn, New York, USA; ${ }^{2}$ NYU Langone Hospital - Brooklyn, Brooklyn, New York, USA

Introduction: The efficacy of MDCT-based-angiography in management of acute stroke and/or emergent-large-vessel-occlusion is well established. However, concern for contrast-induced nephropathy(CIN) especially in patients with major risk factors like Diabetes \& Chronic kidney disease often delays rapid evaluation of ELVO patients. Many published studies report the overall incidence of CIN after administration of IV or IA iodinated contrast and highlight the direct correlation of dose on higher incidence of CIN. None, however, have examined impact of sequential IV-IA bolus for neuroangiographic evaluation on renal function in patients with DM and/or CKD.

Methods: A retrospective study of our 2015-2017 stroke database of 168 patients was conducted to identify all patients with preexisting DM and/or CKD who developed CIN during their hospital course. We also reviewed the prevalence of dehydration (BUN/Cr $<20)$, $\mathrm{CHF}$ and anemia $(\mathrm{Hb}<8 \mathrm{~g} / \mathrm{dL}$ ) for these patients on admission.

Results: For all 168 patients; average IA, IV and cumulative IV-IA contrast (Omnipaque 350) doses within 24 hours were 89.9, 91.7 and $181.6 \mathrm{cc}$ respectively. 68 patients had DM and/or CKD of which 3 developed CIN. Under the definition of $\geq 25 \%$ increase in baseline $\mathrm{Cr}$ within $72-120$ hours of receiving contrast, all 3 had CIN. However, under the definition of $\geq 0.5 \mathrm{mg} / \mathrm{dL}$ increase in Cr within 72 hours, none had CIN. All 3 only had preexisting DM as risk factor and had age appropriate baseline $\mathrm{Cr}$ on admission. The baseline $\mathrm{Cr}$ for each of the 3 patients were $0.82,1.17 \& 0.47 \mathrm{mg} / \mathrm{dL}$ respectively while the elevated $\mathrm{Cr}$ were $1.03,1.17 \& 0.76 \mathrm{mg} / \mathrm{dL}$ respectively. All 3 returned to within baseline by discharge with no mortality or need for hemodialysis.

Conclusions: There is low risk of developing CIN in high risk patients like CKD or DM following rapid sequential dual IV/IA contrast bolus in acute stroke patients and therefore should not delay rapid neuro-angiographic evaluation.

Keywords: Acute Stroke, Angiographic Ct, Mechanical Thrombectomy, Imaging, Endovascular Therapy

Financial Disclosures: The authors had no disclosures.

Grant Support: None. 


\title{
Poster 43
}

\section{Transradial Access: A Feasible and Safe Approach to Neuroendovascular Procedures}

\author{
Waldo R Guerrero, $M D^{1}$, Sudeepta Dandapat, $M D^{1}$, Edgar E Samaniego, $M D^{1}$, \\ Colin P Derdeyn, $M D^{\prime}$, James $D$ Rossen, $M D^{\prime}$, Binbin Zheng-Lin, MD', Santiago \\ Ortega-Gutierrez, $M D, M S^{1}$
}

'University of lowa Hospitals and Clinics, lowa City, lowa, USA

Introduction: The majority of neuroendovascular interventions are done via a transfemoral approach. However, because of complex aortic anatomy or femoral arterial disease, this approach is, at times, not possible. Advantages of radial access include decreased cost and patient preference. Despite these advantages, radial access is rarely employed for neurointerventional procedures. Herein, we describe a variety of cases utilizing a transradial approach as well as describe our technique and periprocedural and major complications.

Methods: We reviewed our institutional database to identify patients who underwent neuroendovascular intervention via a transradial approach over 2 years (September 2015 to September 2017). Records were reviewed for periprocedural and major complications and requirement of radial approach.

Results: 13 cases were identified. The mean age was 70 and $31 \%$ were male. Radial access was performed using a 6 french short-sheath $(7 / 13)$ or Neuromax sheath $(6 / 13)$. A wide variety of procedures were done: 2 diagnostic angiograms follow-ups, 1 AVF Onyx embolization, 2 basilar artery thrombectomies, 2 vertebral artery angioplasties and stenting, 2 subclavian artery angioplasties and stenting, and 2 internal carotid artery angioplasties and stenting, 1 posterior communicating artery coiling, and 1 posterior communicating artery flow diverter embolization. Indications for radial access were: aortic arch/vessel tortuosity for 7 out of 13 patients (54\%), body habitus (BMI above 60 ) for 3 out of 13 patients (23\%), and bilateral severe femoral disease or occlusion for 3 out of 13 patients (23\%). There were no periprocedural and major complications.

Conclusions: For selected patients with difficult femoral access or anatomical variations precluding provision of a stable support for intracranial intervention, the transradial approach is a safe and feasible alternative route of access. We demonstrated that a wide spectrum of neurointerventional cases can be safely performed via a radial approach. It may warrant consideration as a standard method of access in neuroendovascular procedures.

Keywords: Endovascular Therapy

Financial Disclosures: The authors had no disclosures.

Grant Support: None. 


\section{Poster 44}

\section{Usual and Unusual Craniofacial Anomalies Associated with the Duplicate Pituitary Gland-Plus Syndrome}

Navi Nallasamy, $M B B S^{1}$, Virginia Hill, $M D^{1}$

${ }^{1}$ Cleveland Clinic, Cleveland, Ohio, USA

Introduction: Duplication of pituitary gland and associated craniofacial anomalies are rare in occurrence. The objective is to give information on the clinical and imaging findings of a rare case of duplicated pituitary gland with associated midline facial anomalies in a new-born female infant. A literature study of 58 cases of duplicate pituitary gland syndrome reported in the literature is also completed. The usual and unusual craniofacial anomalies associated with this syndrome are further classified.

Methods: The methodology involved case study using clinical results and imaging findings. Then a comprehensive literature survey was completed. A 3-day-old female infant was presented with multiple midline defects which included a nasal pit, midline tubular tongue mass, and a hard and soft palate cleft. An MRI of brain and neck was performed.

Results: MRI of the brain with axial T1 weighted sequence shows a broad, shallow sella turcica with no midline pituitary gland or infundibulum. On the contrary, there are two T1 hyperintense pituitary glands along the lateral borders of the sella. Two separate infundibula extend from these borders. Sagittal T1 weighted post-contrast sequence shows a nodular contour of the hypothalamus with a mass at the floor of the third ventricle. There is no associated enhancement of the mass. The mass likely represents "small, tightly packed nerve cells interspersed by glial cells", as described in multiple case reports. The table shows the unusual and unusual presentations associated with the DPG syndrome.

Conclusions: Duplication of the pituitary gland is a rare entity with less than approximately 60 cases reported in the literature. The associated anomalies may include midline craniofacial defects of the cleft palate, bifid tongue and uvula, nasoseptal mass, and commonly a nasopharyngeal teratoma or dermoid inclusion cyst. A tongue/oral mass, as in this case, has also been previously reported to be associated with the duplicated pituitary gland.

Keywords: Diagnostic Neuroradiology, Imaging, MRI, Cerebral, Head And Neck Malformation Therapy

Financial Disclosures: The authors had no disclosures.

Grant Support: None. 
Poster 45

\section{Surgical Removal of a Retained Microcatheter After Embolization of a Thoracic Spinal Metastasis}

Osama Jamil, $M D^{1}, M$ Yashar Kalani, $M D^{1}$, Min Park, $M D^{2}$

${ }^{1}$ University of Utah, Salt take city, Utah, USA; ${ }^{2}$ University of Virginia

Department of Neurosurgery, Virginia, USA

Introduction: Endovascular techniques can play an essential role in preoperative planning and embolization of vascular tumor. While complications are generally rare, they can occur in any neurointerventional procedure and can be divided into two categories: catheterization related and embolization related. We present the case of a 67 year old male who suffered a catheter fracture during Onyx embolization of a spinal metastasis requiring surgical removal.

Methods: Chart review

Results: A 67-year-old male with a history of metastatic renal cell carcinoma was found to have a T1 spinal metastasis with resulting canal compression. He underwent preoperative endovascular embolization with Onyx using a Marathon microcatheter via the thyrocervical trunk prior to undergoing resection of the lesion the following day . Despite limiting reflux to less than $3 \mathrm{~cm}$, the microcatheter became glued into the vessel. During attempted retrieval with gradual and gentle traction, the microcatheter fractured leaving a remnant of the catheter coiled within the subclavian artery. After discussions with the primary team and the patient, the decision was made to remove the microcatheter via an open surgery due to the patient's prior history of DVTs and hypercoagulable state secondary to the metastatic disease. The catheter was removed successfully via a supraclavicular approach without complication.

Conclusions: Endovascular entrapment of the catheter during embolization procedures are a rare but well described phenomenon in the literature. While there are multiple techniques described for attempted endovascular retrieval of the microcatheter, open surgical retrieval can be a viable option. 1234

Keywords: Embolization, Endovascular Therapy, New Technique

Financial Disclosures: The authors had no disclosures.

Grant Support: None. 
Poster 46

\section{Early Major Recurrence of Coiled Aneurysms: Report of Two Cases}

Mohamad Abdalkader, $M D^{1}$, Jean Raymond, $M D^{2}$, Varun Naragum, $M D^{3}$, Katharine M Cronk, $M D, P h D^{1}$, Thanh N Nguyen, $M D^{1}$

${ }^{1}$ Boston Medical Center, Boston, Massachusetts, USA; ${ }^{2}$ Centre Hospitalier Université de Montréal, Montreal, Canada; ${ }^{3}$ University of Maryland, Baltimore, Maryland, USA

Introduction: Recurrence of an intracranial aneurysm is a known shortcoming of endovascular coiling. It can be due to recanalization and/or regrowth and is typically reported after 6 months of coiling. Although early major recurrence (less than 6 months) is rare, it harbors an impending risk of rupture and needs to be addressed urgently.

Methods: We report two cases of early dramatic regrowth of ruptured aneurysms occurring within 4 months after endovascular coiling. Major aneurysm recurrence has been previously defined as recurrence that would permit introduction of additional coils. We define dramatic aneurysm recurrence as aneurysm recurrence greater than twice the size of the initially visualized aneurysm. We will provide a literature review about the risk factors associated with rapid recurrence after treatment and propose earlier followup for small ruptured aneurysms after coiling.

Results: Thrombus formation within the aneurysmal cavity, hemodynamic stress and turbulence of incompletely treated aneurysm and/or incomplete packing of the aneurysm inflow zone may have contributed in the rapid regrowth in our two cases. In addition, both aneurysms were very small size ( $3 \mathrm{~mm}$ or less) before coiling, presented in a ruptured state and were located at a communicating segment. Both patients underwent successful balloon assisted re-coiling.

Conclusions: Ruptured, small aneurysms, incomplete aneurysm occlusion are common and potential risk factors for dramatic recurrence of intracranial aneurysms. Consideration for earlier short term follow-up after coiling (i.e. 3 months) may be warranted for patients with very small ruptured aneurysms after coiling.

Keywords: Aneurysm, Aneurysm Embolization, Recanalization, Embolization, Coiling

Financial Disclosures: The authors had no disclosures.

Grant Support: None. 
Poster 47

\section{Coil Migration During Endovascular Coiling of Cerebral Aneurysms}

Mohamad Abdalkader, $M D^{1}$, Varun Naragum, $M D^{2}$, Hesham Masoud, $M D^{3}$, Nicholas Tarlov, $M D^{4}$, Alexander M Norbash, MD ${ }^{5}$, Thanh N Nguyen, $M D^{1}$

${ }^{1}$ Boston Medical Center, Boston, Massachusetts, USA; ${ }^{2}$ University of Maryland, Baltimore, Massachusetts, USA; ${ }^{3}$ Suny Upstate Medical University, Syracuse, New York, USA; ${ }^{4}$ Mercy Hospital of Buffalo, Buffalo, New York, USA; ${ }^{5}$ UC San Diego Medical Center, San Diego, California, USA

Introduction: With the widespread use of coil embolization, the periprocedural complications including coil migration remains an important problem. Coil migration during endovascular embolization is an unintended complication where appropriate salvage strategies are important to avoid catastrophic consequences.

Methods: We report a case-series of 4 cases of coil migration occurring during endovascular coiling of intracranial aneurysms.

Results: Four cases of coil migration were identified during endovascular aneurysm coiling from 2007-2017. Management of the migrated coil in the cerebral arteries depends on location of migration (proximal or distal), patient symptoms, the time of migration (during the procedure or delayed) and the presence of associated vessel occlusion. Two of our cases were detected incidentally on follow-up few months after the procedure, no treatment was initiated because of the asymptomatic course of the migration and the distal location. Two cases occurred during the procedure, one case was treated with snare device with no neurological sequela and another case with surgical extraction after non-successful attempt of endovascular extraction. Several factors have been identified in our series associated with coil migration including wide neck aneurysm, low aspect ratio (dome height to neck ratio), small aneurysm, insertion or removal of the last coil, and undersized or short length coils.

Conclusions: Because of the low incidence of coil migration, there is no standard management strategy regarding the best retrieval mechanism of the errant coils. If the patient is asymptomatic, leaving the coil is likely a safer strategy than its retrieval. As endovascular treatment of aneurysms continues to evolve and with the rapid technical advances, the development of new techniques for safe retrieval of migrated coils is important.

Keywords: Coiling, Aneurysm Embolization, Aneurysm, Endovascular Therapy, Aneurysm Embolization

Financial Disclosures: The authors had no disclosures.

Grant Support: None. 
Poster 48

Abstract withdrawn 
Poster 49

\section{Outward Remodeling of the Basilar Artery in a Pre- Aneurysmal Rat Model}

Stacie L Demel, DO, PhD' ${ }^{1}$, Courtney Fisher, $B S^{1}$

${ }^{1}$ Michigan State University, East Lansing, Michigan, USA

Introduction: Cerebral aneurysm formation is due to hemodynamic changes that lead to increased inflammation, smooth muscle cell apoptosis and wall dilatation. Early changes to arterial wall structure contributing to to aneurysm formation are not known. We hypothesized that we would see outward remodeling and increased distensibility of the basilar artery in a novel pre-aneurysmal rat model.

Methods: 18-week-old male spontaneously hypertensive stroke-prone (SHRSP) rats underwent bilateral carotid artery stenosis (BCAS). 2 months post-surgery, animals were sacrificed and basilar arteries were dissected out and prepared for arterial pressure myography. Sodium nitroprusside (10-5M) and EGTA ( $2 \mathrm{mM}$ ) were added to phosphate buffered saline solution (ph 7.4) and warmed to 37 degrees. Lumen diameter and vessel diameter were measured over a range of pressures $(4-140 \mathrm{mmHg}$ ). Wall thickness, wall area and distensibility were calculated. Groups were compared using two-way ANOVA.

Results: SHRSP rats had blood pressures consistently higher than WKY rats and BCAS surgery did not have an effect on blood pressures compared to their respective controls. WKY + BCAS rats had increased outer $(\mathrm{P}=0.001)$ and lumen diameters $(\mathrm{P}=0.0008)$ compared to controls, whereas SHRSP + BCAS and SHRSP controls had similar outer and lumen diameters $(\mathrm{P}=0.55$ and $\mathrm{P}=0.17$, respectively). BCAS resulted in increased distensibility in the normotensive rats, but not the hypertensive rats. Wall thickness and wall area was not different between groups.

Conclusions: There is outward remodeling and increased distensibility of the basilar artery in this novel, pre-aneurysmal rat model in normotensive, but not hypertensive animals. These findings suggest that the BCAS model in normotensive rats provides a physiologically relevant model to study the pathophysiology of early cerebral aneurysm formation.

Keywords: Aneurysm, Basilar

Financial Disclosures: The authors had no disclosures.

Grant Support: None. 


\section{Poster 50}

\section{Changes in Histone Deacetylase mRNA Expression in a Normotensive and Hypertensive Pre-Aneurysmal Rat Model}

Courtney L Fisher, BS ${ }^{1}$, Meredith K Herman, Student ${ }^{1}$, Stacie L Demel, DO/PhD ${ }^{1}$

${ }^{1}$ Michigan State University, East Lansing, Michigan, USA

Introduction: Hypertension is a leading risk factor for intracranial aneurysm (IA) formation. Subsequent rupture of IAs results in subarachnoid hemorrhage $(\mathrm{SAH})$ which carries high morbidity and mortality. Currently, there are no pharmacological treatments available to prevent the progression of IAs. Previously, a genome-wide association study (GWAS) identified a single nucleotide polymorphism (SNP) associated with IA. This SNP is located near histone deacetylase-9 (HDAC-9), FERD3L, and TWIST1. In a separate GWAS, SNPs within the HDAC-9 gene were associated with large vessel atherosclerotic disease and stroke. We developed a novel rodent model of IA formation and measured changes in mRNA expression of HDAC-1, -7, and -9 , as well as nearby genes FERD3L and TWIST1.

Methods: 18-week-old male normotensive Wistar Kyoto (WKY) and hypertensive stroke prone spontaneously hypertensive (SHRSP) rats underwent bilateral carotid artery stenosis (BCAS) or sham surgery. Animals were euthanized 8 weeks post-BCAS, the posterior circulation dissected, and total mRNA extracted for qRT-PCR analysis.

Results: HDAC-1, -7 , and -9 mRNA expression was upregulated in tissue from the posterior circulation in $\mathrm{WKY}+\mathrm{BCAS}$ rats compared to controls $(\mathrm{p}=$ $0.04,0.04,0.04$ ); it was unchanged in SHRSP+BCAS compared to controls. FERD3L and TWIST1 mRNA expression was unchanged in both $\mathrm{WKY}+\mathrm{BCAS}$ and SHRSP+BCAS groups compared to controls.

Conclusions: We have developed a novel rat model of IA formation that reflects changes in HDAC mRNA expression, as hypothesized by previous GWAS studies. Future studies are planned to test the hypothesis that increased expression of HDACs is protective against aneurysm formation.

Keywords: Intracerebral Aneurysm, Aneurysm, Subarachnoid Hemorrhage

Financial Disclosures: The authors had no disclosures.

Grant Support: None. 


\title{
Poster 51
}

\section{Systematic Review and Meta-Analysis of the Angiographic Evolution of Neck Residual (Class II) Aneurysms}

\author{
Rakesh Khatri, MD' ${ }^{\prime}$, Luis Carrete, B.Sc (Neuroscience)' ${ }^{1}$, Mohtashim Arbaab \\ Qureshi, MD', Anantha Ramana Vellipuram, MD' , Salvador Cruz-Flores, MD', \\ Gustavo J Rodriguez, $M D^{1}$, Alberto Maud, $M D^{1}$
}

${ }^{1}$ Texas Tech University Health Sciences Center, El Paso, Texas, USA

Introduction: Coiled intracranial aneurysms are more likely than clipped aneurysms to recur and may require additional intervention. Due to the variable morphology of neck residuals and the lack of natural history studies, the degree to which neck residuals can be considered a favorable outcome after initial treatment is questionable. Our systematic review was aimed to evaluate the existing data on the natural history neck residuals with regards to recanalization rate versus rate of progressive occlusion, and aneurysmal characteristics contributing to these angiographic outcomes.

Methods: The Raymond-Roy Occlusion Classification (RROC) was used in order to define neck residuals in this study. We undertook systematic literature review for treatment of ruptured and unruptured intracranial aneurysms, initial incomplete occlusion, recanalization rates and progressive occlusion of neck residuals. Out of 64 studies analyzed, 18 studies were selected to characterize the angiographic evolution of Class II aneurysms. Of those 18 studies, 12 were utilized for statistical analysis to study the difference between overall recanalization rates compared to progressive thrombosis rates.

Results: A total of 3131 aneurysms were included in the analysis (unruptured $n=1172,37.4 \%$, and ruptured $n=1959,62.6 \%$ ). The average angiographic follow-up after initial treatment was $19.1 \pm 17.3$ months. Upon angiographic follow-up the average percentage of recanalized Class II aneurysms (Class II to Class III) was $19.6 \pm 13.1 \%$ versus the average percentage of progressive thrombosis (Class II to Class I) at $44.2 \pm 24.4 \%(\mathrm{p}=0.03 ; 95 \% \mathrm{CI}=$ 3.02 to 45.99 ). Risk factors associated with recanalization were low volumeembolization ratio at initial treatment, large dome diameter $(>10 \mathrm{~mm})$, wide neck $(\geq 4 \mathrm{~mm})$, and ruptured aneurysm at time of initial treatment.

Conclusions: There is a greater tendency of neck residual (Class II) aneurysms to progress to complete obliteration than worsening to Class III residual status, especially in well packed, small unruptured aneurysms with favorable neck.

Keywords: Aneurysm, Intracerebral Aneurysm, Coiling, Recanalization

Financial Disclosures: The authors had no disclosures.

Grant Support: None. 
Poster 52

\section{Endovascular Treatment of Ruptured Vertebrobasilar Dissecting Pseudoaneurysms Using the Pipeline Flex Embolization Device: Single-Institution Experience}

Waldo R Guerrero, $M D^{1}$, Santiago Ortega-Gutierrez, MD, $M S^{1}$, Minako Hayakawa, $M D^{1}$, Colin Derdeyn, $M D^{1}$, James Rossen, $M D^{1}$, David Hasan, $M D^{1}$, Edgar $A$ Samaniego, $M D^{1}$

${ }^{1}$ University of lowa Hospitals and Clinics, lowa City, lowa, USA

Introduction: Treatment of ruptured posterior circulation dissecting aneurysms is technically challenging with potentially high morbidity and mortality. We sought to assess the safety and feasibility of using a flow diverting device (FDD) and a specific acute antiplatelet aggregation protocol in the management of patients with ruptured dissecting aneurysms.

Methods: Subjects with ruptured dissecting aneurysms treated in our institution during a period of three years were retrospectively identified from a prospective registry. Intraoperative and periprocedural management and complications as well as morbidity and mortality were recorded. Tirofiban maintenance infusion without bolus was administered intravenously (0.1 $\mathrm{mcg} / \mathrm{kg} / \mathrm{min}$ ) immediately following the deployment of the FDD and all patients were loaded with dual antiplatelet immediately post procedure. Clinical follow-up evaluation was performed and modified Rankin Scale (mRS) assessed.

Results: Nine subjects [ 6 males and 3 females with a mean age of 48 (range 25-72)] with ruptured posterior circulation dissecting aneurysms were analyzed. This cohort had 7 dissecting aneurysms (5 vertebral artery and 2 posterior inferior cerebellar artery) and two blister aneurysms with average size of $5 \mathrm{~mm}$ (Range of $2-8 \mathrm{~mm}$ ). Their average World Federation of Neurosurgical Societies score was 2 (range 1-5). Seven patients had an external ventricular drain placed. Two patients went on to have a ventriculoperitoneal shunt. Eight patients received tirofiban infusion without bolus after FDD placement. No intraoperative complications encountered. Two subjects developed asymptomatic/silent intraparenchymal hemorrhage (IPH) found on surveillance noncontrast computed tomography. One subject suffered major IPH few days postoperatively resulting in mortality after additional anticoagulation was added due to patient's severe cardiomyopathy and LVAD. Follow up mRS within 12 months was 0 in three subjects, 1 in three subjects, 2 in one subject, and 4 in one.

Conclusions: Treatment of dissecting pseudoaneurysms with flow diverter device and our suggested antiplatelet aggregation protocol is feasible and potential alternative to other endovascular treatment options.

Keywords: Intracerebral Aneurysm, Flow Diverter, Subarachnoid Hemorrhage, Aneurysm Embolization, Platelet Testing

Financial Disclosures: The authors had no disclosures.

Grant Support: None. 
Poster 53

\section{Outcome of Patients with Middle Cerebral Artery Aneurysms Including Complex Aneurysms Who Underwent Endovascular Repair}

Yahia M Lodi, MD, FAHA, FANA, FAAN. ${ }^{1}$, Varun V Reddy, $M D^{2}$, S Javed, $M D^{3}$, Waleed Elnour, $M D^{4}$, Sumeet Multani, $M D^{4}$, Nishant Runawat, $M D^{5}$, Scott Brehaut, $M D^{6}$, Tony Wong, MD, Ph.D $D^{7}$

'Upstate Medical University, Binghamton/WHS-WMC/MVHS-Health, Binghamton, New York, USA; ${ }^{2}$ Upstate Medical University, Binghamton/UHSWMC/MVHS-Health, Binghamton, New York, USA; ${ }^{3}$ UHS-Wilson Medical Center, Johnsoncity, New York, USA; ${ }^{4}$ UHS-WMC/Upstate Medical University, Binghamton, Johnson City, New York, USA; ${ }^{5}$ Lynchburg General Hospital, Lynchburg, Virginia, USA; ${ }^{6}$ MVHS-Health, Utica, Utica, New York, USA; ${ }^{7}$ UHSWMC/Upstate Medical University, Binghamton/MVHS-Health, Johnsoncity, New York, USA

Introduction: Endovascular repair of the aneurysm continue to gain popularity which has been well excepted by patients. However, middle cerebral artery aneurysms (MCA) are still favored for open craniotomy. Objectives: To evaluate the outcome of patients who underwent endovascular repair of the MCA aneurysm including wide neck and complex aneurysms. Additionally, we like to evaluate the average hospital stay after the treatment.

Methods: Consecutive patients who underwent envovascular repair of the MCA aneurysm from January 2011 to April 2017 analyzed retrospectively. Patients long-term outcome was measure using modified Rankin Scale (mRS).

Results: 22 patients with median age of 64 years (38 to 78) with 24 MCA aneurysms underwent endovascular repair; wide neck and complex anatomy 18 , ruptured 2, symptomatic 12 , recurrent 3 and asymptomatic 7 . Primary coiling was performed in 8 aneurysms. Stent-assisted coiling was intended in 18 but performed in 16 cases. Intra-operative asymptomatic LM2 occlusion in one which was corrected using intra-arterial interline. Clinical events in 2 cases; asymptomatic subarachnoid hemorrhage which resolved in 24 hours and discharged home in 48 hours. Temporal lobe stroke developed in other in primary coiling group with NIHSS 0 , who had significant vascular risk factors and didn't receive antiplatelet. Outcomes: Immediate complete and near complete obliteration of aneurysm achieved in 21 aneurysms and 3 had subtotal obliteration. Median 18 months (6 to 36 months) aneurysm obliteration: complete and near-complete in 18, recurrence in 3 and subtotal in 3 . Recurrent cases underwent treatment to obliteration. Median hospital stay was 1.4 days (1-7 days) excluding 2 ruptured cases. 90 days mRS 0 in 15, mRS 1 in 5 and mRS 2 in one patients.

Conclusions: Our series demonstrates that endovascular repair of MCA aneurysm is associated with short hospital stay and good functional outcome including whose are wide neck and complex in morphology. Further studies are needed. 
Keywords: Aneurysm Embolization, MCA, Stent Assisted, Interventional Neuroradiology, Subarachnoid Hemorrhage

Financial Disclosures: The authors had no disclosures.

Grant Support: None. 
Poster 54

\section{Stent-Assisted versus Lone Coiling in the Treatment of Unruptured MCA Aneurysms Outcome; Dual-Center Experience} Ossama y Mansour, MD PhD FNR'
Anton Valavanis, Is $M D^{3}$

${ }^{1}$ Alexandria university, Stroke and Interventional Neurology Department, Alexandria, Egypt; ${ }^{2}$ Qena University, Qena, Egypt; ${ }^{3}$ Klinik für Neuroradiologie am Universitätsspital Zürich., Zürich, Switzerland

Introduction: Endovascular coil embolization is an increasingly used and continuously evolving method. Simple coiling and stent assisted coiling are 2 methods widely used to treat MCA aneurysms We aimed to review our experience with endovascular treatment of UMCAa either with SAC or LC.

Methods: 52 incidental MCA aneurysms in 50 consecutive patients treated endovascularly from January 2009 to December 2014 in two institutions. We studied the outcome in comparison with the literature.

Results: Angiographic results immediately after embolization showed favorable occlusion in $86.5 \%$ which improved to $92.3 \%$ during long term follow up. We used LC in 30 aneurysms (57.7\%), double catheter technique in 6 aneurysms (11.5\%), catheter assisting technique in 2 aneurysms $(3.8 \%)$ and stent assisted coiling (SAC) in 22 aneurysms (42.3\%). There were 7 thromboembolic events (13.5\%) and two intraoperative perforations $(3.8 \%)$ all without clinical impact. All patients (100\%) had follow- up MRA. There were three recanalizations without retreatment and no bleeding. Progressive occlusion was more frequently reported in SAC treated aneurysms $77 \%$ with $\mathrm{P}<$ 0.05 .

Conclusions: Our study demonstrated that the good clinical and radiological outcome, the low morbidity and the stability of endovascular treatment, make it a good option for treatment for UMCAas. SAC showed a higher rate of progression toward favorable occlusion.

Keywords: Intracerebral Aneurysm, Unruptured, Stent Assisted, Coiling, Angiogram

Financial Disclosures: The authors had no disclosures.

Grant Support: None. 


\title{
Poster 55
}

\section{Y-Stenting of Complex Wide-Neck Intracranial Aneurysms: A Multicenter Experience with the LVIS Jr Device}

\author{
Aldo A Mendez, M.D. ${ }^{1}$, Santiago Ortega-Gutierrez, M.D. ${ }^{1}$, Thanh N Nguyen, M.D. ${ }^{2}$, \\ Ameer E Hassan, D.O. ${ }^{3}$, Guilherme Dabus, M.D. ${ }^{4}$, Vladimir Kalousek, M.D. ${ }^{5}$, Italo \\ Linfante, M.D. ${ }^{4}$, Edgar A Samaniego, M.D. ${ }^{1}$ \\ ${ }^{1}$ University of lowa, lowa City, lowa, USA; ${ }^{2}$ Boston Medical Center, Boston, \\ Massachusetts, USA; ${ }^{3}$ Valley Baptist Medical Center, Harlingen, Texas, USA; \\ ${ }^{4}$ Miami Cardiac and Vascular Institute, Miami, Florida, USA; ${ }^{5}$ University Clinical \\ Hospital Center "Sestre Milosrdnice", Zagreb, Croatia
}

Introduction: Complex wide-neck intracranial aneurysms are challenging to treat. In this study, we report a multicenter experience using the LVIS Jr stent for "Y-stent" assisted coiling embolization of wide-neck bifurcation aneurysms.

Methods: Five centers provided retrospective data on patients who underwent Y-stent assisted coiling embolization for the treatment of wide-neck bifurcation aneurysms using the LVIS Jr device. Technical complications, immediate post-treatment angiographic results, clinical outcomes and imaging follow- up were assessed.

Results: Nineteen patients/aneurysms were treated: 10 basilar tip, 6 middle cerebral artery and 3 anterior communicating artery. Mean aneurysm size was $7.16 \mathrm{~mm}$. Mean dome to neck ratio was $1.23 \mathrm{~mm}$. 36 LVIS Jr stents were successfully deployed without any technical issue. One patient developed basilar artery thrombosis after placement of a WEB device in a basilar tip aneurysm. Y-stenting through a Scepter balloon was performed as a bailout maneuver, however, the patient died due to basilar artery occlusion. Five (27.8\%) periprocedural complications occurred in the 18 patients who survived: one in-stent thrombosis, one posterior cerebral artery (PCA) infarct, one transient aphasia and two retroperitoneal hematomas. Only one patient suffered permanent neurological damage due to a PCA infarct $(6 \%, 1 / 18$ patients). Immediate complete obliteration was achieved in 9 cases, residual neck and aneurysm (RROC Class II-III) in 9 cases. Ten patients had clinical and imaging follow-up (mean of 5.5 months). Complete and partial angiographic occlusion on follow-up was observed in $8(80 \%)$ and $2(20 \%)$ cases respectively. Good functional outcome with a $\mathrm{mRS}(\leq 2)$ was observed in 17 cases. No patients required additional endovascular treatments.

Conclusions: Y-stent assisted coiling of complex wide-neck aneurysms with the LVIS Jr device was technically feasible in this small multicenter case series.

Keywords: Stent Assisted, Aneurysm Embolization, Coiling, Aneurysm,

Financial Disclosures: The authors had no disclosures.

Grant Support: None. 
Poster 56

Outcomes of Endovascular Coiling versus Surgical Clipping
of Unruptured Cerebral Aneurysms in a Large Cohort

Sunil A Sheth, Assistant professor ${ }^{1}$, FNU Komal, Professional trainee ${ }^{1}$, Farhaan $S$ Vahidy, Assistant Professor', Taylor A Daileda, Medical Sutdent ${ }^{2}$, Peng R Chen, Associate Professor ${ }^{1}$, Conrad W Liang, Interventional Neurologist ${ }^{3}$

${ }^{1}$ University of Texas Health Science Center, Houston, Texas, USA; ${ }^{2}$ McGovern Medical School, Houston, Texas, USA; ${ }^{3}$ Fontana Medical Center, Fontana, California, USA

Introduction: Endovascular coiling (EC) has become the preferred treatment over surgical clipping (SC) for unruptured CAs. However, this trend is based on trials for ruptured CAs; data comparing outcomes of unruptured CAs is lacking. Here, we compare the adverse events (AEs) and outcomes of EC vs. SC for unruptured CAs in a large, contemporary cohort.

Methods: Using administrative data on all discharges from acute care hospitals in California (2005-2011) and Florida (2005-2014), we identified patients with unruptured CAs who underwent EC or SC. We compared patient demographics, perioperative (within the same hospitalization) and 30-day AEs and discharge outcomes. We used logistic regression to assess factors associated with AEs and presented the results as OR [95\% CI].

Results: Among 9848 patients with treated unruptured CAs, median age was 60(IQR 51-69), 73\% were female and 62\% were white. 6504(66\%) patients underwent EC and 3344(34\%) SC. Patients treated with EC were more likely to have renal disease ( $3 \%$ vs. $2 \%, \mathrm{p}<0.05)$ and peripheral vascular disease (PVD) $(5 \%$ vs. $3 \%, \mathrm{p}<0.0001)$. Length of stay was shorter for EC ( 2 vs. 4 days, $\mathrm{p}<0.0001$ ). Perioperative AEs were less frequent for EC vs. SC (total $4.2 \%$ vs. $10.6 \%, \mathrm{p}<0.0001)$, including stroke/hemorrhage $(2.2 \%$ vs. $6.5 \%, \mathrm{p}<0.001)$, cardiac complications $(0.5 \%$ vs. $1.0 \%, \mathrm{p}<0.01)$, VP shunt placement $(0.4 \%$ vs. $1.3 \%, \mathrm{p}<0.001)$, EVD placement $(0.2 \%$ vs. $1.7 \%, \mathrm{p}<$ $0.001)$ and death $(0.32 \%$ vs. $0.72 \%, \mathrm{p}<0.01)$. Rates of discharge to skilled nursing or rehab were less for EC $(4.7 \%$ vs. $14.4 \%, \mathrm{p}<0.001)$. In multivariate logistic regression, independent predictors of AEs or death were SC vs. EC (OR $2.4[2.0-2.7])$, advanced age (65-69 vs. < 50, OR 2.0 [1.3-3.0]) and PVD (OR 1.5 [1.1-2.0]).

Conclusions: In this real-world cohort of nearly 10,000 patients with treated unruptured CAs, EC resulted in better outcomes compared to SC, despite greater pre-procedural co-morbidity.

Keywords: Clipping, Coiling, Unruptured, Endovascular

Financial Disclosures: The authors had no disclosures.

Grant Support: None. 


\section{Poster 57}

\section{Radiographic Efficacy of Middle Meningeal Artery Embolization in Treatment of Chronic Subdural Hematoma}

Ryan T Bo, $M D^{1}$, Nathan S Farkas, $M S^{1}$, Igor Teslya, $B A^{1}$, George Selas, $M D^{2}$, Ambooj Tiwari, MD, $M P H^{1}$, David Turkel-Parrella, $M D^{1}$, Karthikeyan Arcot, $M D^{1}$, Jeffrey Farkas, $M D^{1}$

${ }^{1}$ NYU Langone Hospital - Brooklyn Interventional Neuro Associates, Brooklyn, New York, USA; ${ }^{2}$ NYU Langone Hospital - Brooklyn, Brooklyn, New York, USA

Introduction: Chronic subdural hematoma $(\mathrm{cSDH})$ can be associated with slow cognitive decline, co-ordination symptoms and rarely motor-sensory deficits. Open surgical treatment may or may not be always effective or indicated. Some far east operators have studied hypertrophy of Middle Meningeal Artery (MMA) and its embolization for treatment of such recalcitrant lesions. We present our experience of MMA embolization as an earlyadoptive technique for treatment for poor or failed surgical candidates.

Methods: 10 patients diagnosed with unilateral or bilateral cSDH underwent MMA embolization. Size of SDH volume and densities were measured from time of initial discovery on imaging to pre-operative, immediate postoperative, and long-term follow-up. Time between procedure to obliteration was also measured based on follow-up imaging.

Results: Out of 10 patients, 5 patients were diagnosed with recurrent $\mathrm{cSDH}$, and 5 with primary cSDH. 7 patients had bilateral $\mathrm{cSDH}$, and 3 unilateral cSDH. Average volume on admission and pre-operatively were 20.7 and $20.6 \mathrm{cc}$, respectively. MMA embolization was on average performed of 26 days post symptoms onset. Immediate post-op CT was performed an average of 46 hours and showed enhancement of the subdural in $40 \%$ cases. This was associated with greater visualization of their extent with average increase in $\mathrm{SDH}$ volume to $21.2 \mathrm{cc}$. Follow-up CT imaging for these patients was done at an average of 128 days post-procedure. Average volume was down to $13.13 \mathrm{cc}$ with mean reduction of $45 \%$ from presentation. 3 patients were determined to have complete obliteration after 1 year.

Conclusions: MMA Embolization has been shown to have a marked reduction in SDH volume post-operatively and can be used as a curative measure for patients who fail conservative medical management and neurosurgical intervention.

Keywords: Embolization, Endovascular Therapy, SDH, Interventional Neuroradiology, Imaging

Financial Disclosures: The authors had no disclosures.

Grant Support: None. 
Poster 58

\title{
Basilar Artery Stent Assisted Percutaneous Transluminal Angioplasty in Symptomatic Isolated Basilar Artery Stenosis
}

\author{
Sudeepta Dandapat, $M D^{1}$, Waldo Guerrero, $M D^{1}$, Nazan S Aksan, $P h D^{\prime}$, Binbin \\ Zheng, $M D^{1}$, Santiago Ortega Gutierrez, $M D^{1}$, Edgar Samaniego, $M D^{1}$ \\ ${ }^{1}$ University of lowa, lowa City, lowa, USA
}

Introduction: We describe our experience of endovascular management with Basilar Artery - Stent Assisted Percutaneous Transluminal Angioplasty (BA-SAPTA) in Basilar Artery Stenosis (BAS) after failed medical management.

Methods: We retrospectively collected data on all patients with isolated symptomatic BAS who underwent BA-SAPTA at two institutions over a period ranging from 2001-2016 and analyzed it based on location of stenosis - Proximal vs mid - basilar artery.

Results: A total of 44 patients met our inclusion criteria out of which 16 patients had proximal BA-SAPTA [pB], 27 had mid BA-SAPTA [mB] and one patient underwent distal BA-SAPTA. The average age was 64 years with 34 males and 37 whites. 36 patients presented with strokes and rest with TIA. The median NIHSS of patients was 4 (range 1-25). The average degree of stenosis was $84.1 \%$ (range $50-100 \%$ ) for all patients. Stents used: Wingspan 15, Cardiac 21, Enterprise 5; Neuroform 3; balloon angioplasty performed in 31 cases. Aggrastat was used in 22 patients and alteplase in 3 patients during the procedure. Dual antiplatelet therapy with aspirin and Plavix was used in all cases except one case in which aspirin and ticagrelor were used. Periprocedural complications included: 3 (11.1\%) deaths in patients with $\mathrm{mB}$ (brainstem strokes leading to respiratory failure), 4 (25\%) $\mathrm{pB}$ and 5 (18.5\%) $\mathrm{mB}$ patients had new strokes, $6(37.5 \%) \mathrm{pB}$ patients and $1(3.7 \%) \mathrm{mB}$ patient experienced in-stent thrombosis, $1(6.25 \%) \mathrm{pB}$ patient had severe epistaxis, and $1(3.7 \%) \mathrm{mB}$ patient developed femoral artery pseudoaneurysm. Follow up mRS 0-2 was seen in $13(81.25 \%) \mathrm{pB}$ and 15 (55.56\%) mB patients.

Conclusions: BA-SAPTA is high morbidity and mortality procedure for treatment of BAS. Patients with proximal BA-SAPTA tend to do better than mid BA-SAPTA, possibly due to "snow-ploughing" effect. The results should be interpreted with caution due to its retrospective nature and small sample size.

Keywords: Basilar, Atherosclerosis, Intra Caranial Stenosis, Stenting, Angioplasty

Financial Disclosures: The authors had no disclosures.

Grant Support: None. 


\section{Poster 59}

\section{Endovascular Recanalization of Intracranial Stenosis in Patients with Recurrent or Progressive Symptoms Despite Medical Management}

Roberta Santos, $M D^{1}$, Pedro Aguilar-Salinas, $M D^{9}$, John J Entwistle, $D O^{9}$, Adnan $\mathrm{H}$

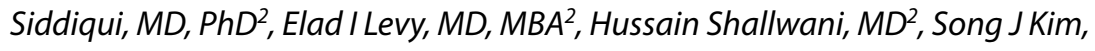
$M D^{3}$, Diogo C Haussen, $M D^{3}$, Raul G Nogueira, $M D^{3}$, Demetrius Lopes, $M D^{4}$, Ahmed Saied, $M D^{4}$, Tudor G Jovin, $M D^{5}$, Ashutosh Jadhav, $M D^{5}$, Limaye Kaustubh,

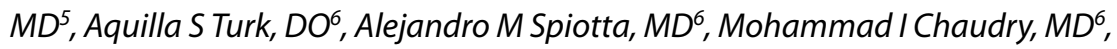
Raymond D Turner, $M D^{6}$, Leonardo B Brasilience, $M D^{7}$, Travis M Dumont, $M D^{7}$, Jacob Cherian, $M D^{8}$, Peter Kan, $M D^{8}$, Eric Sauvageau, $M D^{9}$, Ricardo A Hanel, $M D$, $P h D^{1}$, Amin Aghaebrahim

${ }^{1}$ Baptist Neurological Institute, Jacksonville, Florida, USA; ${ }^{2}$ Department of Neurosurgery and Toshiba Stroke Research Center, University at Buffalo, Buffalo, New York, USA; ${ }^{3}$ Department of Neurology, Grady Memorial Hospital / Emory University School of Medicine, Atlanta, Georgia, USA; ${ }^{4}$ Department of Neurosurgery, Rush University Medical Center, Chicago, Illinois, USA; ${ }^{5}$ Department of Neurology, University of Pittsburgh Medical Center, Pittsburgh, Pennsylvania, USA; ${ }^{6}$ Department of Neurosurgery, Medical University of South Carolina, Charleston, South Carolina, USA; ${ }^{7}$ Division of Neurosurgery, University of Arizona, Tucson, Arizona, USA; ${ }^{8}$ Department of Neurosurgery, Baylor College of Medicine, Houston, Texas, USA; ${ }^{2}$ Lyerly Neurosurgery / Baptist Health, Baptist Neurological Institute, Jacksonville, Florida, USA

Introduction: The optimal management of intracranial arterial stenosis is unclear, particularly in patients who have failed medical management. We now report a multicenter real-world experience of endovascular recanalization of intracranial atherosclerotic stenosis refractory to aggressive medical therapy.

Methods: Retrospective multicenter case series of consecutive endovascularly treated patients presenting with symptomatic (TIA or stroke) intracranial stenosis who had failed medical therapy were identified. All of the patients were considered to be at high-risk category with unstable symptomatic intracranial stenosis and progression or recurrence of symptoms despite best medical management.

Results: 101 patients presented with recurrent TIAs $(n=41)$ or recurrent or progressive strokes $(\mathrm{n}=60)$ and were treated in 8 stroke centers from August 2009 to May 2017. All patients were treated either with dual-antiplatelet therapy or anticoagulation and all of them had statin therapy prior to recurrence or progression of their symptoms. There were 2 periprocedural perforations which resulted in death. Periprocedural stroke occurred in 3 patients and 3 patients had symptomatic intraparenchymal hemorrhage secondary to reperfusion injury. The all-cause mortality rate at discharge was $8.9 \%$ and no mortality was recorded within the first 90 days beyond discharge. At the 90 -day follow-up, 7 patients had TIAs and 2 patients (3\%) had 
ipsilateral ischemic strokes. The 90 -day cumulative stroke rate was $6.7 \%$ and $77.5 \%$ of patients had $\mathrm{mRS}$ of 2 or less.

Conclusions: Endovascular recanalization of unstable intracranial atherosclerotic stenosis in patients who have failed medical therapy is feasible and safe.

Keywords: Intra Caranial Stenosis, Intracranial Stenosis Stenting And Angioplasty, Endovascular Therapy

Financial Disclosures: The authors had no disclosures.

Grant Support: None. 


\title{
Poster 60
}

\section{Safety and Efficacy of Eptifibatide in the Context of Hyperacute or Acute Neuroendovascular Stenting}

\author{
Igor Teslya, $B A^{1}$, Ryan TBo, MD' , Danielle Crotty, Pharm. $D^{2}$, Ting Zhou, MD², \\ David Turkel-Parrella, $M D^{1}$, Ambooj Tiwari, $M D M P H^{1}$, Jeffrey Farkas, $M D^{1}$, \\ Karthikeyan Arcot, $M D^{1}$
}

${ }^{1} \mathrm{NYU}$ Langone Hospital- Brooklyn Interventional Neuro Associates, Brooklyn, New York, USA; ${ }^{2}$ NYU Langone Hospital- Brooklyn, Brooklyn, New York, USA

Introduction: Eptifibatide (Integrilin) has been demonstrated to improve clinical outcomes in both intracoronary and carotid artery stenting, when administered perioperatively. This evidence promotes the investigation of eptifibatide's role in neuroendovascular stenting.

Methods: 38 patients between 2013-2017 underwent intracranial stenting with eptifibatide administration within 24 hours of procedure. Cumulative and average eptifibatide dosages were determined for all patients. Peri and post-procedural bleeding complications were defined as intercranial hemorrhage $(\mathrm{ICH})$, symptomatic intercranial hemorrhage $(\mathrm{sICH})$, and peripheral bleeding (retroperitoneal, access site bleeding, GI bleeding). Final Thrombolysis in Cerebral Infarction (TICI) scores as well as modified Rankin Scales (mRS) at discharge were also collected. Comparisons of these outcomes were made between patients with high-dose Integrilin and low-dose Integrilin, which we defined as above or equal to and below $0.75 \mathrm{mcg} / \mathrm{kg} /$ min, respectively. A similar comparison was performed for patients who received above and below calculated dosage of median cumulative dose ( 0.71 $\mathrm{mg} / \mathrm{kg}$ )

Results: Of all 38 patients, 7 (21.8\%) patients were found to have intracerebral hemorrhage, with 3 of these patients showing symptoms. Additionally, 6 patients (18.7\%) experienced peripheral bleeding complications. Mean eptifibatide dosage was determined to be $0.77 \mathrm{mcg} / \mathrm{kg} / \mathrm{min}$. Analysis of the primary endpoint of all-cause bleeding complications yielded no significance between high-dose and low-dose Integrilin $(\mathrm{p}>0.05)$. However, the incidence of sICH was significantly greater in patients receiving an average dosage at $0.75 \mathrm{mcg} / \mathrm{kg} / \mathrm{min}$ or higher $(\mathrm{p}<0.05)$. Furthermore, angiographic assessment revealed that more patients who receive a cumulative dosage of $0.71 \mathrm{mg} / \mathrm{kg}$ or greater achieved a TICI score of $2 \mathrm{c}-3$ in comparison to those who achieved TICI $2 \mathrm{~b}(\mathrm{p}<0.05)$.

Conclusions: Usage of eptifibatide for patients undergoing neurovascular stenting at higher average dosages may result in a higher incidence of symptomatic ICH, however higher cumulative dosages may improve angiographic outcomes.

Keywords: Angioplasty, Antiplatelet, Coagulation, Drugs Side Effects, Medical Management

Financial Disclosures: The authors had no disclosures.

Grant Support: None. 


\title{
Poster 61
}

\section{DAPT Administration Timing and Effective Reperfusion in Patients with Large Vessel Occlusion Treated with PTA}

\author{
Takeshi Yoshimoto, Department of Neurology', Hiroshi Yamagami, Division of \\ Stroke Care Unit ${ }^{1}$, Kazutaka Sonoda, Division of Stroke Care Unit ${ }^{1}$, Tetsu Satow, \\ Department of Neurosurgery', Jun C Takahashi, Department of Neurosurgery', \\ Masafumi Ihara, Department of Neurology ${ }^{1}$, Kazunori Toyoda, Cerebrovascular \\ Medicine
}

${ }^{1}$ National Cerebral and Cardiovascular Center, Osaka, Japan

Introduction: The efficacy of percutaneous transluminal angioplasty (PTA) in acute ischemic stroke (AIS) due to arteriosclerotic intracranial arterial occlusion remains unclear. We aimed to examine the impact of administration timing of dual antiplatelet therapy (DAPT) on emergent intracranial PTA with or without stenting.

Methods: Among 306 AIS patients undergoing endovascular therapy (EVT) in our hospital between December 2010 and June 2017, we retrospectively examined patients treated with PTA for intracranial artery occlusion. Patients were divided into 2 groups according to the administration timing of DAPT, before or after the first PTA (pre-PTA and post-PTA group). The incidence of effective reperfusion (TICI2b/3), good clinical outcome (mRS 0-2 after 3 months), and symptomatic intracranial hemorrhage (SICH) were compared between 2 groups. SICH was defined as a low intensity signal on MRI T2* images with any neurological deterioration within 36 hours after EVT.

Results: This study included 40 patients ( $74 \pm 11$ years, 26 men), and 18 patients in pre-PTA group. Pre-PTA group had lower NIHSS at admission (12 vs $19, \mathrm{p}=0.04)$, more atherosclerotic occlusion ( $89 \%$ vs $50 \%, \mathrm{p}=0.02$ ) than post-PTA group. Use of IV rt-PA ( $44 \%$ vs $50 \%, \mathrm{p}=0.76$ ), additional stenting ( $56 \%$ vs $23 \%, \mathrm{p}=0.08$ ) were not different. High rate of effective reperfusion $(89 \%$ vs $50 \%, \mathrm{p}=0.02)$ were acquired in pre-PTA group and good clinical outcome tended to be more frequent in pre-PTA compared with post-PTA group $(50 \%$ vs $23 \%, \mathrm{p}=0.10)$. The incidence of SICH ( $6 \%$ vs $14 \%, \mathrm{p}=0.61$ ) was not different between two groups. In multivariable analysis, pre-PTA DAPT was independently associated with effective reperfusion (aOR, 6.67; 95\% CI, 1.08-62.7; $\mathrm{p}=0.04$ ).

Conclusions: DAPT before PTA can be related to effective reperfusion in AIS with intracranial artery occlusion.

Keywords: Intracranial Stenosis Stenting And Angioplasty, Ischemic Stroke, Endovascular Therapy

Financial Disclosures: The authors had no disclosures.

Grant Support: None. 


\section{Poster 62}

\section{Triage to a Comprehensive Stroke Center Based on CT Perfusion Imaging}

Amin Aghaebrahim, Vascular Neurologist ${ }^{1}$, Eric Sauvageau, Director

Cerebrovascular', Pedro Aguilar-Salinas, Research Associate', Gustavo M Cortez, MD Candidate' ${ }^{1}$, Ricardo A Hanel, Director Cerebrovascular ${ }^{1}$

'Baptist Neurological Institute Lyerly Neurosurgery / Bapist Health, Jacksonville, Florida, USA

Introduction: Use of Computed Tomographic Perfusion (CTP) imaging at a referring hospital may help triage and shorten door-to-puncture time for patients with acute ischemic stroke.

Methods: We conducted a single center retrospective review of a prospectively maintained database of consecutive ischemic stroke patients transferred to our center for consideration of endovascular therapy. Patients were divided into two groups. Group 1 consisted of patients transferred from a facility where CTP (using automated RAPID software) was routinely performed and group 2 consisted of patients transferred from a facility that did not perform perfusion imaging.

Results: We identified a total of 132 patients all of whom were transferred to our center from April of 2014 to April of 2017. There were no differences in baseline characteristics between two groups. A total of 34 patients were transferred from a facility with RAPID CTP (Group 1) and 98 were transferred from a facility with no CTP (Group 2). Door-to-puncture time was significantly lower for patients in Group 1 compared with Group 2 (median [IQR], 12 [8-16] minutes and 48.5 [32.8-71.8] minutes, $\mathrm{p}<.001$ ). Despite having additional imaging in Group 1, there was no difference in door-in and door-out times at the referring facilities compared with group 2 .

Conclusions: We found that triaging from a primary stroke center with CTP ability significantly reduced door-to-puncture time as compared with transferred patients with no perfusion imaging without any significant delay in transfer process.

Keywords: Ischemic Stroke, Ct Perfusion, Acute Ischemic Stroke Intervention, Door To Groin Puncture, Mechanical Thrombectomy

Financial Disclosures: The authors had no disclosures.

Grant Support: None. 
Poster 63

Abstract withdrawn 


\section{Poster 64}

\section{The Impact of Ultrarapid Sequential Contrast Bolus for IV/IA Neuroangiographic Evaluation on Renal Function}

\section{Ryan Bo, $M D^{1}$}

${ }^{1}$ NYU Langone Hospital Brooklyn Interventional Neuro Associates, Brooklyn, New York, USA

Introduction: Although common practice in comprehensive stroke centers, community centers still have delays in obtaining rapid CTAs without baseline renal labs for acute stroke patients. This can lead to valuable loss of 30-60 min in urgent evaluation of these cases and has been proven by multiple studies that this can mean the difference between high-functional and poor functional outcomes. We therefore decided to investigate the overall effect of ultra rapid sequential contrast boluses for IV and IA angiographic evaluation of acute strokes and it's effect on final renal function.

Methods: A retrospective review of a comprehensive stroke center database was performed. 68 patients were categorized by baseline renal function at $72-120 \mathrm{~h}$ and renal function at discharge. Relevant angiographic, clinical, and lab data was collected. Outcomes reported included creatinine trends, $\mathrm{BUN}$ trends, and BUN/Cr ratio trends.

Results: Using $\mathrm{Cr}$ as the only measure, $69 \%$ of patients had overall decrease or no change in $\mathrm{Cr}$ at discharge while $31 \%$ had an increase. However, only $16.35 \%$ had increase in $\mathrm{Cr}$ in the first 72 hours while $83.65 \%$ had no change or decrease in Cr. This trend was the contrarian to the trend in BUN and $\mathrm{BUN} / \mathrm{Cr}$ ratio for patients at discharge. 58\% reported increase in both BUN and BUN/Cr from admission to discharge. However, only 56\% in the BUN group and $49 \%$ in the BUN/Cr group had increase in the first 72 hours. Therefore throughout the hospitalization there was an equivocal change in renal function secondary to contrast administration. This was controlled for severity of disease using $\mathrm{mRS}$ at discharge.

Conclusions: True impact of high-dose contrast administration for neuroangiographic evaluation is poorly understood. CIN or AKI in the acute setting may be overtly simplistic. Delay due to simplistic lab like Cr is costly and should be discouraged in any hospital.

Keywords: Vascular Imaging, Drugs Side Effects, Endovascular Therapy

Financial Disclosures: The authors had no disclosures.

Grant Support: None. 
Poster 65

\section{A Functional Outcome Model for a Telestroke-Guided Tissue Plasminogen Activator Treatment of Stroke Patients}

Leanne E Brechtel, Medical Student ' , Jordan Gainey, Student' ', Thomas Nathaniel, $P h D^{1}$

'University of South Carolina School of Medicine Greenville, Greenville, South Carolina, USA

Introduction: The success of telestroke systems to extend stroke-care expertise into rural and underserved areas has been reported. Evidence of clinical outcomes associated with the use of tissue-type plasminogen activator has been documented, but evidence of functional outcome studies in telestroke-guided tissue-type plasminogen treatment are lacking. This issue was investigated in this study.

Methods: Data from a stroke registry of 307 acute ischemic patients treated with intravenous rtPA in a telestroke network was analyzed. A new functional outcome model was developed to asses rtPA treatment outcome using a combination of visual basic and multivariate logistic models. Differences in functional outcomes between patients that received rtPA and those did not receive rtPA were determined. A partial proportional-odds model was developed for common odd ratio based on cumulative logits for all functional outcome variables. The significance level of all analysis was set to a probability level of $\mathrm{p}<0.05$.

Results: In a telestroke-guided rtPA treatment of stroke patients, age was significantly $(\mathrm{P}<0.0001$, OR 0.902$)$ associated with improved functional outcome. Stroke patients with a medical history of obesity $(\mathrm{P}=0.002$, OR 4.016), and a previous history of stroke $(\mathrm{P}=0.024$, OR 2.765) were associated with improved functional outcomes. The odds of an improved functional outcome was higher for stroke patients with systolic blood pressure $(\mathrm{P}=0.012$, OR $3.135)$ as the initial vital sign. Moreover, patients with initial lower NIH stroke scale scores $(\mathrm{P}<0.001$, OR 0.911$)$ were associated with improved functional outcome.

Conclusions: Modified Rankin Scale distribution and other measures have been used to measure clinical outcomes in rtPA-treated patients. The development of a new functional outcome model provides a new approach to improve the efficiency of telestroke and increase of rtPA for stroke patients.

Keywords: Ischemic Stroke

Financial Disclosures: The authors had no disclosures.

Grant Support: None. 


\title{
Poster 66
}

\section{Does Changing Technique During Mechanical Thrombectomy Improve Recanalization Rates?}

\author{
Brendan Eby, Presenter ', Akash Kansagra, Coauthor ${ }^{7}$
}

${ }^{1}$ Washington University in St. Louis, St. Louis, Missouri, USA

Introduction: Higher rates of successful recanalization correlate with improved outcomes following mechanical thrombectomy, with current data demonstrating no difference between stent retriever and aspiration catheter in either recanalization rates or clinical outcomes. When initial thrombectomy attempts fail, neurointerventionalists must decide between continuing with the initial device versus introducing an alternate device. This study investigates whether changing devices provides improved recanalization rates over continuing with the initial device.

Methods: Retrospective chart review of all mechanical thrombectomies performed at a single tertiary care academic hospital between January 2013 and October 2016 was performed. Cases were grouped by initial device technique (stent retriever, aspiration catheter). Cases initiated with a combination of both techniques were excluded. Main technical outcome was radiographic recanalization (TICI $2 \mathrm{~B}$ or 3 ).

Results: Of 152 thrombectomy cases reviewed, 16 did not demonstrate large vessel occlusion by angiography, 12 had technical limitations precluding thrombectomy, and 32 initially utilized a combination of stent retriever plus aspiration catheter; therefore, these were excluded. Of 92 remaining cases, 44 (48\%) were initiated with stent retriever, and 48 (52\%) with aspiration catheter. First pass provided recanalization in $15(34 \%)$ with stent retriever and 22 $(46 \%)$ with aspiration catheter $(\mathrm{p}=0.29)$. Of cases requiring multiple passes, device change was utilized in 6 of $29(21 \%)$ stent retriever cases and 15 of 24 $(63 \%)$ aspiration catheter cases $(\mathrm{p}=0.0042)$. In the stent retriever group, eventual recanalization was achieved in 4 of $6(67 \%)$ cases utilizing a device change, compared with 14 of $23(61 \%)$ without change $(\mathrm{p}=1.00)$. In the aspiration catheter group, eventual recanalization was achieved in 10 of 15 (67\%) cases utilizing a device change, compared with 6 of 9 (67\%) without change $(\mathrm{p}=1.00)$.

Conclusions: Among thrombectomy cases who fail first pass recanalization, final recanalization rates are comparable between cases that implemented a device change and those that continued with the initial device.

Keywords: Acute Ischemic Stroke Intervention, Recanalization, Stentretriever, Ischemic Stroke, Mechanical Thrombectomy

Financial Disclosures: The authors had no disclosures.

Grant Support: None. 
Poster 67

\title{
Effect of Antihypertensive Medications on rt-PA in Stroke Patients; Improved or Poor Outcomes?
}

\author{
Tyler R Fleming, Student' ${ }^{1}$, Benjamin Averkamp, Student ${ }^{1}$, James Sullivan, \\ Student ${ }^{1}$, Thomas Nathaniel, $P h D^{1}$ \\ 'University of South Carolina School of Medicine Greenville, Greenville, South \\ Carolina, USA
}

Introduction: Many studies have reported that antihypertensive medications reduce the severity of stroke or increase the rate of a favorable outcome in rt-PA-treated patients, while others reported worse outcomes. These discrepancies may be attributed to differences in the clinical characteristics of stroke patients. This issue was the focus of our study.

Methods: In 1,141 stroke patients from a stroke registry of data collected between January 2010 to June 2016, the effect of antihypertensive (antiHTN) medication on stroke patients treated using rt-PA was determined. Comparison of improved functional outcomes between rt-PA treated and non-treated groups with antiHTN medication history was determined using univariate analysis while multivariable models were used to adjust for the effect of confounding variables.

Results: Multivariate binary logistic regression analysis with adjustments made for known risk factors and group differences showed a significant association between several medical history factors and functional ambulation in acute ischemic stroke (AIS) patients who have a history of antiHTN use and did or did not receive rt-PA. Factors influencing functional ambulation in patients who did not receive rt-PA included NIHSS score $(\mathrm{OR}=1.159, \mathrm{p}=$ $0.002)$, risk of mortality GWTG $(\mathrm{OR}=0.840, \mathrm{p}=0.005)$, history of atrial fibrillation $(\mathrm{OR}=2.456, \mathrm{p}=0.004)$, and history of previous stroke $(\mathrm{OR}=$ $0.504, \mathrm{p}=0.022)$. Factors influencing functional ambulation in patients who did receive rt-PA included NIHSS score $(\mathrm{OR}=1.094, \mathrm{p}=0.051)$, history of $\mathrm{CHF}(\mathrm{OR}=0.385, \mathrm{p}=0.035)$, and history of previous TIA $(\mathrm{OR}=0.302, \mathrm{p}=$ $0.017)$. Factors influencing functional ambulation in all rt-PA treated patients regardless of antiHTN use included NIHSS score $(\mathrm{OR}=1.125, \mathrm{p}=0.003)$, history of carotid artery stenosis $(\mathrm{OR}=3.641, \mathrm{p}=0.037)$, history of previous TIA $(\mathrm{OR}=0.344, \mathrm{p}=0.007)$, and history of antiplatelet medication use $(\mathrm{OR}=$ $1.887, \mathrm{p}=0.024)$.

Conclusions: In an AIS population treated with antiHTN medication, several clinical variables can be used to determine the inclusion or exclusion of rt-PA therapy that have a significant impact on the functional ambulation of the patient.

Keywords: Acute Ischemic Stroke Intervention, Acute Stroke, Thrombolytics, Decision Analysis

Financial Disclosures: The authors had no disclosures.

Grant Support: None. 


\section{Poster 68}

\section{Comparison of $6 \mathrm{~mm}$ and 3-4 $\mathrm{mm}$ Diameter Stent Retrievers for MT. Is Bigger Better?}

Ameer E Hassan, DO, FAHA, FSVIN ${ }^{1}$, Christina Sanchez, $B A^{2}$, Adnan I Qureshi, $M D^{3}$

${ }^{1}$ UTSCHA - Valley Baptist Medical Center, Harlingen, Texas, USA; ${ }^{2}$ Valley Baptist

Medical Center, Harlingen, Texas, USA; ${ }^{3}$ Zeenat Qureshi Stroke Institute,

Rockford, Illinois, USA

Introduction: There is controversy regarding the optimal size of stent retriever for achieving timely recanalization in acute ischemic stroke patients.

Methods: To determine the relationship between stent retriever diameter, procedure time, rates of recanalization, and functional independence among AIS patients undergoing MT. We analyzed data from consecutive AIS patients treated with MT derived from a prospective database. Baseline demographic and clinical characteristics, NIHSS score on admission and discharge, intracranial hemorrhage occurrence, and mRS at discharge were analyzed. Thrombolysis In Cerebral Infarction (TICI) scale was used to grade pre and post procedure angiographic recanalization. Procedural time was defined by the time interval between microcatheter placement and recanalization. We compared the rates of thrombectomy attempts, complete recanalization (TICI grade of 3), and functional independence (defined by mRS 0-2) between patients treated with $6 \mathrm{~mm}$ and 3-4 mm diameter stent retrievers.

Results: Total of 230 AIS patients (mean age $71.8 \pm 12.5$; $46.6 \%$ women) were treated with stent retrievers. MT was performed with a $6 \mathrm{~mm}$ diameter stent retriever in 107 patients and 3 or $4 \mathrm{~mm}$ diameter stent retriever in 123 patients. There were no statistically significant differences in demographics or baseline characteristics, or admission NIHSS score between the two groups. There was a trend towards a fewer number of thrombectomy attempts required with a $6 \mathrm{~mm}$ diameter stent retriever $(\mathrm{p}=0.06)$. There was a higher rate of complete recanalization in patients treated with $6 \mathrm{~mm}$ diameter stent retriever compared with 3 or $4 \mathrm{~mm}$ diameter stent retriever ( $72 \%$ vs $57.7 \%$ $\mathrm{p}=0.02)$. There was no statistically significant difference in rates of functional independence between the two groups $(24.3 \%$ vs $25.2 \% \mathrm{p}=0.84)$ at discharge.

Conclusions: Among AIS patients undergoing MT, use of a $6 \mathrm{~mm}$ diameter stent retriever was associated with a higher rate of complete recanalization and lower number of thrombectomy attempts compared with 3-4 mm diameter stent retrievers.

Keywords: Acute Ischemic Stroke Intervention, Endovascular Therapy, Revascularization, Stentretriever, Recanalization

Financial Disclosures: Consultant, Speaker, Proctor and Steering Committee for Medtronic; Consultant and Speaker for Stryker; Consultant and Speaker for GE Healthcare; Consultant and Speaker for Microvention; Consultant and Speaker for Penumbra

Grant Support: None. 
Poster 69

\title{
Impact of Residual Stenosis after Successful Reperfusion in the STRATIS Registry
}

\author{
Diogo C Haussen, $M D^{1}$, Osama O Zaidat, $M D^{2}$, Nils Mueller-Kronast, $M D^{3}$, Dileep $R$ \\ Yavagal, $M D^{4}$, Jeffrey $L$ Saver, $M D^{5}$, Ali Aziz-Sultan, $M D^{6}$, Raul G Nogueira, $M D^{1}$, \\ David S Liebeskind, $M D^{7}$ \\ ${ }^{1}$ Emory University School of Medicine/Grady Memorial Hospital, Atlanta, \\ Georgia, USA; ${ }^{2}$ St. Vincent Mercy Hospital, Toledo, Ohio, USA; ${ }^{3}$ Advanced \\ Neuroscience Network, Tenet, Florida, USA; ${ }^{4}$ University of Miam, Miami, \\ Florida, USA; ${ }^{5}$ University of California Los Angeles, Los Angeles, California, \\ USA; ${ }^{6}$ Brigham and Women's Hospital, Boston, Massachusetts, USA; \\ ${ }^{7}$ University of California Los Angeles, Los Angeles, California, USA
}

Introduction: The impact of residual stenosis after thrombectomy is unclear. We analyzed the data from a large prospective registry of patients that underwent thrombectomy within 8 h of last normal with NIHSS $\geq 8 / \leq 30$ (STRATIS) to evaluate the significance of residual stenosis after stent retriever thrombectomy in successfully reperfused patients.

Methods: The STRATIS cohort Arterial Occlusive Lesion(AOL) scoring system at the end of the case was evaluated by the imaging core lab. Final AOL $0-1$, mTICI $0 / 1 / 2 a$, and vasospasm were excluded. Post-thrombectomy residual stenosis (PTRS, defined as final AOL-2 at the original occlusion site after retriever thrombectomy, with or without subsequent angioplasty and/or stenting) versus AOL-3 status was compared in successfully reperfused (mTICI 2b-3) patients.

Results: 43 PTRS patients were compared to 676 AOL-3. Mean age and frequency of comorbidities were not statistically different between groups, with the exception of less atrial fibrillation in the PTRS group ( $18 \%$ vs $38 \% ; \mathrm{p}=0.01$ ). NIHSS, systolic blood pressure, and ASPECTS were similar between groups. Posterior circulation occlusion was more common in the PTRS group (18\% vs $4 \% ; \mathrm{p}<0.001$ ). The use of IV-tpa and the time from onset-to-puncture were similar between arms, however time from puncture-to-reperfusion was longer in the PTRS group $54 \pm 36$ minutes vs $42 \pm 25$ minutes in the AOL-3 $(\mathrm{p}<0.01)$. First-pass reperfusion was less common ( $46 \%$ vs $66 \%$; $=0.001$ ) and the number of passes was higher $(2.1 \pm 1.2$ vs $1.7 \pm 1.1 ; \mathrm{p}=0.02)$ in PTRS patients. Parenchymal hematomas were similar in the PTRS vs AOL-3 groups ( $2.3 \%$ vs. $2.5 \% ; \mathrm{p}=1.00)$. The rates of functional independence (mRS0-2) $(60 \%$ vs $59 \% ; p=1.00)$ and mortality $(7.9 \%$ vs $14.1 \%$; $=0.34$ ) were similar between the PTRS vs AOL-3 arms at 90 days.

Conclusions: In the STRATIS registry, residual stenosis at the end of stent-retriever thrombectomy was associated with longer time to reperfusion, lower chances of first-pass reperfusion, and higher number of passes. Residual stenosis was not observed to impact clinical outcome.

Keywords: Mechanical Thrombectomy, Acute Stroke, Atherosclerosis, Stentretriever

Financial Disclosures: The authors had no disclosures.

Grant Support: None. 


\title{
Poster 70
}

\section{Active Reperfusion Hemorrhage During Mechanical Thrombecomy}

\author{
Diogo C Haussen, $M D^{1}$, Ivan C Ferrerira, $M D^{2}$, Clara Barreira, $M D^{1}$, Francesco \\ Diana, $M D^{3}$, Simone Peschillo, $M D^{3}$, Jonathan A Grossberg, $M D^{1}$, Fernando Diogo \\ Barbosa, $M D^{2}$, Raul $G$ Nogueira, $M D^{1}$ \\ ${ }^{1}$ Emory University School of Medicine/Grady Memorial Hospital, Atlanta, \\ Georgia, USA; ${ }^{2}$ Hospital Santa Lucia, Brazil; ${ }^{3}$ Policlinico Umberto I/Sapienza \\ University, Roma, Italy
}

Introduction: Symptomatic intracranial hemorrhage not uncommonly occurs after mechanical thrombectomy and impacts clinical outcome. Reperfusion injury with active extravasation may be identified intra-procedurally and potentially arrested.

Methods: This was a retrospective analysis of cases of reperfusion injury with active perforator extravasation during thrombectomy in three tertiary care centers.

Results: Six patients were identified. Mean age was $65 \pm 9$ years, $50 \%$ male, NIHSS was $15 \pm 6$, platelets level $193,000 \pm 49,000$, systolic blood pressure $163 \pm 33 \mathrm{mmHg}$, and ASPECTS 7.6 \pm 1.3 . Two patients were taking aspirin and none anticoagulation. One patient had received thrombolysis (and another only thrombolytic bolus). There were three MCA M1, one ICA terminus, and one vertebrobasilar junction occlusion. Three patients had anterior circulation tandem occlusions. Stroke etiology was large artery atherosclerosis $(\mathrm{n}=$ $2)$, intracranial atherosclerosis $(n=2)$, cardioembolic $(n=1)$, cervical dissection $(\mathrm{n}=1)$. Mean time from onset to puncture was $6.0 \pm 2.5$ hours and procedural time $1.7 \pm 0.7$ hours. Intravenous heparin was administered in all patients for a mean dose of $4500 \pm 2100$ units and four patients received additional intravenous abciximab. All tandem cases had the cervical lesion addressed first. Three patients were found to bleed after retriever thrombectomy, one after aspiration+retriever, one after intracranial angioplasty, and another after the neck was revascularized. Five lenticulostriates and one paramedian pontine artery were involved. The bailout included use of protamine (5/6), balloon guide catheter (3/6) or compliant balloon inflation plus coiling of targeted vessel (1/6). Blood pressure was dropped in (3) patients with elevated levels. All patients had cessation of bleeding at the end of the procedure with parenchymal hemorrhage type 1 in one case and type 2 in five. Four patients had modified Rankin score of 4 and 2 were dead at 90 days.

Conclusions: Active hemorrhage post reperfusion typically involved perforators in patients with intraprocedural administration of antithrombotics. Although cessation of bleeding was achieved in all cases, clinical outcomes were grim.

Keywords: Acute Ischemic Stroke Intervention, Hemorrhage

Financial Disclosures: The authors had no disclosures.

Grant Support: None. 


\title{
Poster 71
}

\section{CT Angiography Causes Time Delays and Potential Additional Risks That May Make It an Unnecessary Test for Acute Ischemic Stroke Treatment Algorithm}

\author{
Ashish Kulhari, M.D. ${ }^{1}$, Audrey Arango ${ }^{1}$, Siddhart Mehta', Laura Suhan', Sara \\ Strauss' ${ }^{1}$ Rebekah Amarini' ', Sonia Ahmed' , Amrinder Singh' ', Spozhmy Panezai', \\ Jawad Kirmani, $M D^{1}$ \\ 'JFK Stroke \& Neurovascular Center, Seton Hall Hackensack Meridian School \\ of Medicine, Edison, New Jersey, USA
}

Introduction: Most Stroke Centers in U.S. do vascular imaging (mostly CT angiography) on all acute stroke cases on presentation even if the neurological examination is suggestive of large vessel occlusion. CT angiography puts patient at risk for extra radiation, contrast exposure and delays thrombectomy which affects patients final outcomes. At our institute, we do not routinely get CT angiography on all patients who clinically have large vessel occlusion (LVO) and are within the time window for thrombectomy . Our goal in this study was to see if obtaining a CTA causes delay in recanalization.

Methods: Retrospective analysis of all acute stroke neurointerventions done at our University affiliated community hospital from Jan -Dec 2016 was performed. Patients were divided in two groups: CTA vs No CTA. Door to recanalization times for these groups were calculated and compared.

Results: Total 40 patients underwent endovascular therapy for acute LVO's in 2016. "CTA" group included 30 patients and "No CTA" group included 10 patients. 55\% (22) patients were male. $90 \%$ (36) cases were anterior circulation LVO's. Mean Door to Recanalization time for "CTA" group was 140 minutes (SD 20.84) and for "No CTA" was 179 minutes (SD 15.15). p value < 0.0001 .

Conclusions: Our results depict that Door to Recanalization times are prolonged when CT Angiography is done prior to endovascular therapy. CTA itself utilizes the substantial amount of time but analysis of CTA and decision making delays the neurointervention too. We agree that in complicated cases, CTA could be very useful. We conclude that CTA is an unnecessary test prior to acute neurointervention in patients where clinical examination strongly suggest LVO. * Due to insufficient space for supporting authors, we included authors in Title*

Keywords: Acute Ischemic Stroke Intervention

Financial Disclosures: The authors had no disclosures.

Grant Support: None. 


\section{Poster 72}

\section{Effective Use of Balloon Guide Catheter in Reducing Incidence of Mechanical Thrombectomy Related Distal Embolization}

\section{Dong Hoon Lee, $\mathrm{Dr}^{1}$}

'Department of Neurosurgery, St. Vincent's Hospital, The Catholic University of Korea, Suwon, Korea, Republic of

Introduction: The clinical benefit of endovascular stroke therapy has been demonstrated in several prospective randomized trials. However, in a relevant percentage of patients, mechanical thrombectomy bears the risk of causing new infarction in initially unaffected vascular territories through thrombus fragmentation and migration of clot debris. The goal of this study was to evaluate the use of the balloon guide catheter (BGC) to effectively achieve flow arrest and thrombus aspiration during the intervention in order to avoid distal embolization.

Methods: A retrospective study was performed in 139 patients between October 2010 and May 2016, to analyze occlusions in the middle cerebral artery (MCA) or internal carotid artery (ICA) by using a stent retriever with a BGC $(n=73)$ or a non-BGC $(n=66)$. The following data were collected: patient age and gender, along with risk factors. Data on procedure time, number of passes, and angiographic findings were also collected. The final reperfusion score was rated based on the TICI grading scale. Successful recanalization was defined as TICI 3 or $2 \mathrm{~b}$.

Results: A total of 139 patients underwent mechanical thrombectomy with the stent retriever. Of the 139 patients, 73 (52.5\%) underwent placement of a BGC. The numbers of passes were similar between the two groups. The procedure time $(99 \pm 49.4$ minutes vs. $124 \pm 72.2$ minutes; $\mathrm{P}=0.02)$ and the time from onset of symptoms to procedure end (302 \pm 102 minutes vs. $357.2 \pm 136.1$ minutes; $\mathrm{P}=0.009$ ) were shorter in the BGC group. TICI 3 or $2 \mathrm{~b}$ recanalization scores were higher in the BGC group compared to the nonBGC group (63/73, $86.3 \%$ vs. $48 / 66,72.7 \%$; odds ratio (OR), 0.6 ; $95 \%$ confidence interval $(\mathrm{CI}), 0.2-1.4 ; \mathrm{P}=0.04)$. Importantly, distal embolization was less frequent in the BGC group (5/73, $6.8 \%$ vs. $21 / 66,31.8 \%$; OR, 6.3 ; $95 \% \mathrm{CI}$, 2.2-18.0; $\mathrm{P}<0.001$ ).

Conclusions: The risk of distal embolization was significantly decreased with the use of a BGC.

Keywords: Acute Ischemic Stroke Intervention

Financial Disclosures: The authors had no disclosures.

Grant Support: None. 
Poster 73

\section{Experience of Intraarterial Thrombectomy in Patient with Cancer-Related Cerebral Infarction Over 5 years}

Dongwhane Lee, Clinical fellow, MD' ${ }^{1}$, Hyuk-sung Kwon, clinical fellow' ${ }^{1}$ Da-eun Jeong, clinical fellow' ${ }^{1}$, Min Hwan Lee, clinical fellow', Jong S. Kim, Professor ${ }^{1}$, Sun

U. Kwon, Professor ${ }^{1}$

${ }^{1}$ Department of neurology, University of Ulsan, Asan Medical Center, Seoul, Korea, Republic of

Introduction: Patients with current malignancy (CM) are excluded from intravenous thrombolysis for many causes of hematologic problem and history of recent surgery. Intraarterial thrombectomy (IAT) is now considered the standard therapy in acute ischemic stroke (AIS) patients. We aimed to investigate and compare the characteristics of IAT in AIS with CM and remote malignancy $(\mathrm{RM})$

Methods: We retrospectively reviewed twenty-seven patients with CM or RM of 207 patients treated with IAT from January 2012 to June 2017. We compared the clinical data including cancer associated factors, IAT related factors, laboratory and radiologic findings, and prognosis (modified Rankin scale, mRS).

Results: Thirteen patients had CM and the others had RM. CM group revealed younger, more leukocytosis and higher $\mathrm{C}$-reactive protein. $\mathrm{D}$-dimer was higher in CM group, but it was not statistically significant. Adenocarcinoma was the most common pathology of any cancer type (14 (66.7\%) of 21). Moreover, CM group had more ICA occlusion (12 (92.3\%) vs $5(35.7 \%), \mathrm{p}=0.004)$, more prevalent in hospital stroke $(10(76.9 \%)$ vs 2 $(14.2 \%), \mathrm{p}=0.002)$, less atrial fibrillation $(3(23.0 \%)$ vs $10(7.1 \%), \mathrm{p}=0.021)$, more concomitant balloon angiography $(6(46.1 \%)$ vs $1(35.7 \%), \mathrm{p}=0.033)$ and IAT (6 (46.1\%) vs $1(7.1 \%), \mathrm{p}=0.033)$. However, no significant difference were identified between two groups regarding 3 -month good functional outcome $(\mathrm{mRS}<3)(2(15.3 \%)$ vs $5(35.7 \%), \mathrm{p}=0.385)$

Conclusions: CM group seems to have more poorer outcome than RM group, it is seen to be less effective than the general outcome of IAT. Nevertherless, it didn't show significant differences. It was thought to be small numbers of RM group. Also, there is no reason to hesitate to perform IAT for patient with active cancer in AIS. Further study including healthy controls that have never had cancer will be needed.

Keywords: Acute Ischemic Stroke Intervention, Stentretriever, Endovascular Therapy, Intra-Arterial Therapy, Recanalization

Financial Disclosures: The authors had no disclosures.

Grant Support: None. 


\title{
Poster 74
}

\section{Intracranial Hemorrhage following Thrombectomy in Patients Presenting with a Large Vessel Occlusion and an Initial NIHSS of $\leq$ to 8}

\author{
Joshua Lukas, $M D^{1}$, Vasu Saini, $M D^{1}$, Priyank Khandelwal, $M D^{1}$, Omar Alkhatib, \\ $M D^{1}$, Ashutosh Mahapatra, $M D^{1}$, Kunakorn Atchaneeyasakul, $M D^{1}$, Dileep \\ Yavagal, $M D^{\prime}$ \\ 'University of Miami, Miami, Florida, USA
}

Introduction: Few studies have assessed the safety and efficacy of acute endovascular therapy for patients presenting with a LVO and an NIHSS of $\leq 8$. To date, no definite conclusion has been reached leaving the controversial question of whether or not to treat this population of patients. Therefore an in depth analysis of safety and outcomes is necessary to aid in selection and appropriate treatment.

Methods: We examined a retrospective cohort of consecutive patients $>18$ years-of-age with acute ischemic stroke undergoing mechanical thrombectomy at an academic center from 2/7/2015 to 6/29/2017. Our primary outcome was defined as TICI $2 \mathrm{~b}-3$ reperfusion and our safety outcomes were defined as the presence of symptomatic intracranial hemorrhage (sICH) or any hemorrhage within 36 hours post-thrombectomy. The patient was considered symptomatic if they had an increase in NIHSS of $>4$ within 24-36 hours post-thrombectomy.

Results: 26 patients with initial NIHSS of $\leq 8$ were analyzed. The mean age was $65.6+/-17.7$ years (range, 28-90). The mean initial NIHSS was $5.85+$ 2.13. $21 / 26$ (81\%) had successful reperfusion (TICI score of 2b-3). 6/26 (23\%) received intra-arterial TPA and $3 / 26(12 \%)$ received intra-arterial verapamil. $8 / 26(30.8 \%)$ of the patients with NIHSS $\leq 8$ had an ICH compared to $21 / 156$ (13.5\%) with NIHSS $>8$ (RR: $2.29 ; \mathrm{p}=0.0207 ; 95 \%$ CI: 1.1344 to 4.6053$)$. Of those with NIHSS of $\leq$ to $8,3 / 26(12 \%)$ had a symptomatic intracranial hemorrhage (sICH) and $8 / 156(5 \%)$ of the patients with NIHSS $>8$ had a sICH (RR: $2.25 ; \mathrm{P}=0.2037 ; 95 \% \mathrm{CI}: 0.6380$ to 7.9346 )

Conclusions: Our findings demonstrated a significant increased rate of ICH in patients with initial NIHSS of $\leq 8$, however fewer patients were symptomatic as compared to patients with NIHSS $>8$. Further investigation is necessary to identify specific factors contributing to this outcome.

Keywords: Treatment, Invention, Acute Ischemic Stroke Intervention

Financial Disclosures: The authors had no disclosures.

Grant Support: None. 
Poster 75

\section{Role of Thin-Sliced Reformatted CT Imaging for Acute Ischemic Stroke Patients, Do We Need CT Angiography Before Deciding for Acute Neurointervention?}

Siddhart Mehta, M.D. ', Ashish Kulhari', Amrinder Singh ${ }^{1}$, Sonia Ahmed', Sara Strauss', Spozhmy Panezai ', Jawad Kirmani, $M D^{7}$

1JFK Stroke \& Neurovascular Center, Seton Hall Hackensack Meridian School of Medicine, Edison, New Jersey, USA

Introduction: Thin-sliced reformatted non-contrast CT head are not widely used to detect the thrombus. The purpose of this study was to investigate whether thin-sliced reformatted non-contrast CT scans could be reliably used to detect and measure size of the thrombus size in patients with acute ischemic stroke due to LVO and therefore serve as a potential substitute of CT Angiogram (CTA). Success of this paradigm could reduce stroke decision algorithm to nonenhanced CT scan before a code neurointervention is activated; hence saving time and contrast.

Methods: Comprehensive prospective analysis of patients who underwent acute endovascular intervention for LVOs at a community based, university affiliated comprehensive stroke center during two year period (Jan 2015-Dec 2016) was done. The raw data of non-enhanced CT scans and CTAs were collected. All raw data were reconstructed with thin slices of 0.625 $\mathrm{mm}$ using standard GE software. Vessel diameter and clot length were measured on both CT and CTA. (5-mm maximum intensity projections of the thin slices on CT and axial/coronal projections on CTA). Each patient's CT and CTA were paired for length and diameter in millimeters. Paired two sample hypothesis test was run on SPSS. Digital subtraction angiography (DSA) images was reviewed to confirm the clot location.

Results: There were 926 patients who presented with acute ischemic stroke during the specified time period. Of those 99 were LVOs that received endovascular treatment of which, 37 had both CT and CTA done and had intracranial lesions. Mean clot length measured was 13.99 (SD 5.34) on thin slices CT and 14.18 (SD 5.64) on CTA. Vessel diameter measured was 2.83 (SD 0.54) on thin sliced reformatted CT and 2.55 (SD 0.51) on CTA. There was no significant difference in estimated clot length done on CT as compared with CTA. $(\mathrm{P}=0.601)$, CI with lower limit of -0.92 to and upper limit of 0.54. There was significant difference in estimated vessel diameter on CT as compared with CTA. ( $\mathrm{P}<0.0001)$, CI with lower limit of 0.17 and upper limit of 0.38. DSA confirmed clot location in all cases.

Conclusions: Thin-sliced reconstructions of standard cranial nonenhanced CT raw data can be reliably used to detect and measure the thrombus size in LVOs. It does slightly overestimate the vessel diameter but still making intervention planning possible. Larger multicenter trials are needed to validate our data. 
* Due to insufficient space provided for supporting authors, we included authors in Title*

Keywords: Acute Ischemic Stroke Intervention

Financial Disclosures: The authors had no disclosures.

Grant Support: None. 
Poster 76

\title{
A Proposed Cocktail for Fluctuating Small Vessel Infarction to Prevent Deterioration
}

\author{
Siddhart Mehta, M.D. ${ }^{1}$, Ashish Kulhari ${ }^{1}$, Anna Barminova ${ }^{1}$, (Peter) Yong-Bum \\ Song', Sara Strauss', Spozhmy Panezai', Jawad Kirmani, $M D^{1}$ \\ 1JFK Stroke \& Neurovascular Center, Seton Hall Hackensack Meridian School \\ of Medicine, Edison, New Jersey, USA
}

Introduction: Small vessel infarcts, accounting for approximately one fourth of all ischemic strokes, pose a unique challenge in terms of acute management. As with other types of infarcts, the guidelines for lacunar strokes recommend the use of intravenous tissue plasminogen activator provided the patients present within the accepted time window and have no contraindications. However, unlike large vessel infarcts, patients presenting with small vessel infarcts are not candidates for intra-arterial thrombectomy due to challenges posed by vessel caliber and the acute angles at which small vessels tend to branch from parent vessels. This raises questions regarding treatment options for these patients in the acute setting, particularly in those patients with demonstrating fluctuation and deteriorating symptoms.

Methods: Comprehensive evaluation of a patient who underwent acute medical management for deteriorating small vessel infarction in a dedicated Neurocritical Care Unit (NCCU) at a community based, university affiliated comprehensive stroke center was done.

Results: A 61-year-old African American right handed female with hypertension, diabetes and hyperlipidemia presented to the emergency department with acute onset slurred speech, right facial droop and right sided weakness that began the morning prior. Imaging revealed acute left paramedian pontine infarction with no underlying visualized stenosis/occlusion of the basilar artery. Initial treatment was our standard stroke protocol which includes intravenous fluids, and oral agents: Aspirin, Clopidogrel and high dose Atorvastatin. After fluctuation of symptoms cilastazole was added as results of platelet function Assays were pending. On the second day of admission, the patient began having episodic deterioration of her presenting symptoms. More aggressive management was initiated including Neosynephrine infusion to maintain cerebral perfusion pressure. There was no improvement and further deterioration ensued to NIHSS of 12 . Verapamil and magnesium infusion for vasodilatory/neuroprotective effect, low dose Heparin infusion for anticoagulation, and Albumin pushes for plasma volume expansion. The patient was closely monitored with an arterial line and central venous pressure monitoring in NCCU. Patient showed rapid stabilization with significant improvement in her right sided weakness and dysarthria. At her two-month follow up visit, the patient had no neurological deficits.

Conclusions: This case illustrates a unique approach in achieving stabilization and improvement in patients with fluctuating symptoms in acute small vessel infarction. Due to the beneficial results attained, further studies into 
using a multimodal approach is warranted utilizing this method. * Due to insufficient space for supporting authors, we included authors in Title*

Keywords: Ischemic Stroke, Treatment

Financial Disclosures: The authors had no disclosures.

Grant Support: None. 
Poster 77

\title{
Safety of Acute Intracranial Stenting for Treating Posterior Circulation Strokes in the Stentriever Era
}

\author{
Siddhart Mehta, M.D. ', Ashish Kulhari', Amrinder Singh', Sonia Ahmed', Spozhmy \\ Panezai ', Jawad Kirmani ${ }^{1}$ \\ 1JFK Stroke \& Neurovascular Center, Seton Hall Hackensack Meridian School \\ of Medicine, Edison, New Jersey, USA
}

Introduction: Multiple recent positive endovascular trials have proven mechanical thrombectomy as standard of care for large vessel occlusion (LVO) of the Anterior Circulation (AC). None of these endovascular studies included patients with Posterior Circulation (PC) strokes. However, there are few trials evaluating the benefit of clot retrieval in posterior circulation strokes. Best treatment options for PC LVO are a focus of intense investigation. We explore the safety and early efficacy of acute intracranial stents in acute PC LVO.

Methods: We conducted a retrospective analysis of patients who underwent acute endovascular intervention for posterior circulation LVOs at a community based, university affiliated comprehensive stroke center during a five year period (2012-2017). Patients with acute intracranial stenting were included. Complications were noted and categorized in major versus minor. Initial NIHSS, discharge NIHSS and discharge mRS were abstracted. A severity matched comparison of discharge mRS to composite 90 day mRS of recent multi-centered prospective randomized control trials (MPRCT) for acute endovascular treatment of acute ischemic stroke involving LVOs was made.

Results: There were 2,980 patients who presented with acute ischemic stroke during the pre-specified time period. Of those 310 were posterior circulation strokes; 16 underwent endovascular treatment using acute stenting. These were compared with 1,386 patients with proximal anterior circulation strokes included in the recent MPRCTs. The mean age was 62 (SD 12.60). Mean initial NIHSS was 21 (SD 11.31). Mean change in NIHSS was 9.1 (SD 8.94). Mean discharge NIHSS was 11.2 (SD 10.09). Mean discharge mRS was 3.3 (SD 1.30). There were no major or minor complications. Comparative group analysis revealed no statistically significant difference when outcomes were compared to MPRCTs.

Conclusions: The safety and early efficacy of acute intracranial stenting for posterior circulation LVO compares well with those recently achieved by the trials evaluating the treatment for anterior circulation strokes. A prospective randomized trial should be attempted elucidate efficacy of acute stenting versus stentrievers for posterior circulation LVO. * Due to insufficient space for supporting authors, we included authors in Title *

Keywords: Acute Ischemic Stroke Intervention

Financial Disclosures: The authors had no disclosures.

Grant Support: None. 


\section{Poster 78}

\section{Direct Aspiration Catheter Fracture and Retrieval During Neurothrombectomy}

Pouria Moshayedi, $M D, P h D^{1}$, Ashutosh P Jadhav, $M D, P h D^{1}$

${ }^{1}$ Department of Neurology, University of Pittsburgh Medical Center, Pittsburgh, Pennsylvania, USA

Introduction: Application of direct aspiration catheters has revolutionized acute stroke care and has led to significant improvement in clinical outcome with a good safety profile. Catheter fracture and retention is a rare but potentially devastating complication.

Methods: Here we present two cases of acute stroke complicated by aspiration catheter fracture and retention. Foreign body retrieval was successfully achieved using snare devices.

Results: Successful catheter retrieval and revascularization was achieved in both cases. The stenosis or tortuosity of vascular anatomy appears to be the probable contributor to catheter breakage by anchoring the catheter with resultant fracture at the constraint point from catheter withdrawal tensile stress.

Conclusions: This report describes application of snare devices in retrieving broken catheter during thrombectomy in the anterior and posterior circulation, and therefore presents a technique that can be safely utilized to address catheter breakage complicating thrombectomy in different vascular anatomic locations.

Keywords: Acute Ischemic Stroke Intervention, Mechanical Thrombectomy

Financial Disclosures: The authors had no disclosures.

Grant Support: None. 
Poster 79

\title{
Direct Aspiration (ADAPT) and Stent Retriever Thrombectomy for Acute Stroke: Systematic Review in 5441 Patients
}

\author{
Christopher T Primiani, $B S^{1}$, Maxim Mokin, $M D, P h D^{2}$, Adnan H Siddiqui, MD, \\ $P h D^{3}$, Aquilla S Turk, $D O^{4}$, Elad I Levy, $M D^{3}$, Italo Linfante, $M D^{5}$ \\ 'University of South Florida Morsani College of Medicine, Tampa, Florida, USA; \\ ${ }^{2}$ University of South Florida, Tampa, Florida, USA; ${ }^{3}$ University at Buffalo, Buffalo, \\ New York, USA; ${ }^{4}$ Medical University of South Carolina, Charleston, South \\ Carolina, USA; ${ }^{5}$ Miami Cardiac and Vascular Institute, Miami, Florida, USA
}

Introduction: The advent of modern devices for thrombectomy has revolutionized endovascular stroke therapy. The two most common approaches to thrombectomy currently include: direct aspiration first pass technique (ADAPT) or primary stent retriever thrombectomy. We aimed to compare radiographic and clinical outcomes in patients with acute stroke from large vessel occlusion (LVO) treated with these two approaches.

Methods: Using the PubMed database, we searched for studies that included patients with acute ischemic stroke from LVO treated with direct aspiration (ADAPT) or primary stent retriever thrombectomy. The literature search included 305 studies, excluding 133 manuscripts. Statistical analysis was performed on 38 publications with a total of 3028 patients that underwent thrombectomy using a stent retriever device and 29 publications with a total of 2413 patients that underwent thrombectomy using ADAPT. We compared the proportions of patients with successful recanalization, favorable functional outcomes, intracranial hemorrhage, mortality rates, and occurrence of distal embolization in the two treatment groups by calculating chisquared and confidence intervals for odds ratios.

Results: 5541 patients from 67 publications were included in the results. There was no difference in age ( $\mathrm{p}=0.6221,95 \%$ CI $-1.75-2.90)$, NIHSS ( $\mathrm{p}=$ $0.1140,95 \%$ CI $-2.31-0.25)$, the use of intravenous thrombolysis $(\mathrm{p}=0.6374$, 95\% CI -17.09-27.69), or location of occlusion (internal carotid artery, middle cerebral artery or posterior circulation; $p=0.3829$ ) between the two groups. We found no difference in rates of successful recanalization TICI $2 \mathrm{~b} / 3$ (83.2\% with ADAPT vs. $75.9 \%$ with primary stent retriever; $\mathrm{p}=0.4044$, 95\% CI -0.071-0.17) or good clinical outcome at 3 months defined as mRS $0-2(46.7 \%$ with ADAPT vs. $46.5 \%$ with primary stent retriever; $\mathrm{p}=0.9550$, 95\% CI -0.092-0.087).

Conclusions: According to our systematic review, endovascular treatment with direct aspiration (ADAPT) and primary stent retriever thrombectomy approaches results in similar radiographic and clinical outcomes in patients with acute stroke from LVO.

Keywords: Acute Ischemic Stroke Intervention, Endovascular Therapy, Mechanical Thrombectomy, Ischemic Stroke

Financial Disclosures: The authors had no disclosures.

Grant Support: None. 


\section{Poster 80}

\section{Endovascular Treatment of Acute Ischemic Strokes $>6 \mathrm{~h}$ from Last Known Well: Community Hospital Cohort Outcomes}

Pankajavalli Ramakrishnan, $M D, P h D^{1}$, Diane Forbes, MIS, RRA, RT(R)(VI), RDMS', Adam Akari, BS candidate ${ }^{2}$, Wolfgang Leesch, $M D^{1}$, J. Frank Sanderson, $M D^{1}, M$. O'Brien Gossage, RN, BSN, SCRN ${ }^{1}$, Dean Kostov, $M D^{1}$

${ }^{1}$ Riverside Regional Medical Center, Newport News, Virginia, USA;

${ }^{2}$ Christopher Newport University, Newport News, Virginia, USA

Introduction: Endovascular treatment of acute ischemic stroke has been established as the standard of care in patients with anterior circulation emergent large vessel occlusion (ELVO) who present within 6 hours. Recent data indicates that patients with ELVO presenting beyond this window with clinical-imaging mismatch demonstrated benefit following treatment with mechanical thrombectomy.

Methods: Here we present the results of the endovascular treatment of patients presenting with ELVO in the anterior or the posterior circulation beyond 6 hours $(n=23)$, and compare their clinical and radiographic outcomes in comparison to those patients presenting within 6 hours from last known well (LKW) between 2015-2017 $(n=52)$. Patients were selected for treatment in the beyond $6 \mathrm{~h}$ window with imaging using ASPECTS, MRI, and/or collaterals on post contrast CT.

Results: The baseline characteristics were as follows: median age (74 vs. 76), NIHSS (16 vs. 18.5), LKW (507 min vs. $58 \mathrm{~min}$ ), ASPECTS (7 vs. 8) in the LKW $>6 \mathrm{~h}$, or unwitnessed onset/ wake up stroke patients ( LKW $>6 \mathrm{~h}$ ) vs. in those LKW $<6 \mathrm{~h}$, respectively. ELVO was identified in the proximal MCA M1 segment (52\% vs. $59.6 \%$ ), and in the ICA Terminus (30\% vs. $19.2 \%$ ) in the LKW $>6 \mathrm{~h}$, and the LKW $<6 \mathrm{~h}$ cohort, respectively. TICI $2 \mathrm{~b}$ or 3 reperfusion was achieved in $78.3 \%$ in the LKW $>6 \mathrm{~h}$ cohort, and $67.3 \%$ in the LKW $<6$ h cohort. sICH rates were $8.7 \%$ vs. $11.5 \%$ in the LKW $>6$ h group vs. LKW $<6 \mathrm{~h}$. Modified Rankin Scores at $90 \mathrm{~d}$ were collected and will be analyzed once the 2017 data is gathered.

Conclusions: We demonstrate that in a community based hospital, the endovascular treatment of patients with ELVO presenting beyond $6 \mathrm{~h}$ or with an unwitnessed onset, or deficit discovery upon wake up is comparable to those patients presenting $<6 \mathrm{~h}$ from LKW.

Keywords: Acute Ischemic Stroke Intervention, Mechanical Thrombectomy

Financial Disclosures: The authors had no disclosures.

Grant Support: None. 


\title{
Poster 81
}

\section{Improvement of Acute Cognitive Deficits in Mild Ischemic Stroke is Associated With TPA Administration}

\author{
David Rosenbaum-Halevi, $M D^{1}$, Andrew Bursaw, DO², Rahul R Karamchandani, \\ $M D^{1}$, Susan E Alderman, $R N^{1}$, Joshua I Breier, PhD', Farhaan S Vahidy, $M D^{1}$, Sean I \\ Savitz, $M D^{1}$ \\ 1 Department of Neurology, University of Texas Health Science Center, \\ Houston, Texas, USA; ${ }^{2}$ San Antonio Military Medical Center, San Antonio, \\ Texas, USA; ${ }^{3}$ Center for Clinical Neurosciences, University of Texas Health \\ Science Center, Houston, Texas, USA
}

Introduction: Cognitive deficits as result of Acute Ischemic Stroke (AIS) are a known, but underappreciated phenomena. TPA administration could help minimize cognitive sequelae in AIS thus warranting assessment of cognitive deficits in the acute phase.

Methods: 46 patients with AIS were prospectively enrolled. TPA was administered based on institutional guidelines. Cognitive battery included the California Verbal Learning Test(CVLT-II), Rey-Osterrieth Complex Figure test (ROCF), Weschler Memory Scale(WMS-III), Symbol Digit Modality Test(SDMT), Trail-Making Tests A\&B, FAS test, and the Montreal Cognitive Assessment(MoCA). Battery was repeated at 90 days. Baseline cognition was determined by age, years of education (YrE), history of cognitive impairment, and extent of white matter lesions on MRI as defined by Fazekas score (Faz-score).

Results: 25 patients completed the study. There was no significant difference in age, sex, Faz-score, or YrE between TPA/No-TPA groups. Two sample t-test for Z-score improvement in WMS-III (follow-up - baseline) $\pm \mathrm{SD}$, was significant in TPA $0.74 \pm 0.77$ vs No-TPA $-0.02 \pm 0.83(\mathrm{P}=0.0395)$. Logistic regression model for predicting normal status in WMS-III at follow-up correcting for Faz-score and age showed OR 8.96, CI 0.98-82.12 $(\mathrm{P}=0.053)$ in favor of treatment group. Z-score for improvement (follow-up - baseline) by Faz-score in treatment group was significant for improvement on ROCFDR, Faz score 0/1 $(\mathrm{N}=4)-0.51 \pm 0.61$ Faz-score $2-5(\mathrm{~N}=3) 0.60 \pm 0.46(\mathrm{P}=0.0484)$, and with trend toward improvement on WMS-III, Faz-score $0-1(\mathrm{~N}=4) 0.40$ \pm 0.37 , Faz-score $2-5(\mathrm{~N}=3) 1.43 \pm 0.8(\mathrm{P}=0.0696)$.

Conclusions: TPA administration is associated with improvement in cognitive functioning at 90 days. Patients with limited cognitive reserve are more likely to show improvement in cognition after TPA. Screening for cognitive deficits in the acute phase of AIS for the purpose of TPA administration is feasible and should be incorporated into clinical decision making for TPA administration. Additionally, tests of cognitive function should be included in outcome measures.

Keywords: Acute Ischemic Stroke Intervention, TPA, MRI, Decision Analysis, Cerebrovascular Disease

Financial Disclosures: The authors had no disclosures.

Grant Support: NIH T32 Grant 


\section{Can Real-World Experience from a High Volume Center Confirm Existing Strong Evidence for Thrombectomy Devices?}

Jan Rothaupt, Dr. ${ }^{1}$, Lars Krause, Dr. ${ }^{1}$, Florian Stoegbauer, Prof. ${ }^{1}$, Bernd Tombach, Prof.', Stephan Lowens, Dr. ${ }^{1}$

${ }^{1}$ Klinikum Osnabrück, Osnabrück, Germany

Introduction: In the light of a growing body of strong evidence for mechanical thrombectomy it becomes more and more important to put these results in perspective with real-world data.

Methods: A total of 371 patients with large intracranial vessel occlusion were treated in a single center with mechanical thrombectomy since 2012 in an all-comer scenario. Emergency setting and treatment related parameters were monitored constantly and thoroughly over this 5 year period and were used to improve hospital-internal processes and thus patient outcomes. These outcomes are compared to published data from the ARTESp study in order to evaluate the relevance of this study in real world (1).

Results: During the period of 2012-2015 Tici2b/3 could be achieved in 92.5\% compared to $84 \%$ in the ARTESp study, whereas sICH rates were slightly higher ( $4 \%$ compared to $2 \%$ in ARTESp) as well as overall mortality rate with $12.1 \%$ (all causes) compared to $7 \%$ in ARTESp. Updated numbers including the more recent experience will be presented in order to show the improvement over 5 years.

Conclusions: Morbidity and mortality rates are slightly higher as to be expected in the absence of inclusion/exclusion criteria to select a certain patient cohort, but the overall results are encouraging and show the validity of clinical studies such as ARTESp beyond their controlled area of investigation. Patient outcomes can be improved significally beyond average even in an all-comer setting when compared to clinical study data, if thorough monitoring is conducted for all relevant parameters and process improvement is driven based on these facts (2).

Keywords: Ischemic Stroke, Stentretriever, Mechanical Thrombectomy, TICI, Acute Ischemic Stroke Intervention

Financial Disclosures: The authors had no disclosures.

Grant Support: None. 
Poster 83

\section{Pilot Study of Safety of Direct Mechanical Thrombectomy Without Prior Brain CT in LVO Stroke During Cardiac Catheterization}

Vasu Saini, $M D^{1}$, Samir Sur, $M D^{1}$, Priyank Khandelwal, $M D^{1}$, Brian Snelling, $M D^{1}$, Mauricio Cohen, $M D^{1}$, Eric C Peterson, $M D^{1}$, Dileep R Yavagal, $M D^{1}$

'University of Miami Miller School of Medicine, Miami, Florida, USA

Introduction: Large vessel occlusion (LVO) stroke is uncommon but potentially devastating complication during cardiac catheterization (CC) procedures. IV tPA is contraindicated in this group of patients due to therapeutic heparinization. With the arterial access that is already in place, a cerebral angiogram can definitively diagnose a LVO prior to MT. Therefore a brain CT and CT angiogram may not be necessary to safely proceed to MT and adds to the time to reperfusion without a definite benefit. Hence, we adopted a protocol of directly proceeding with cerebral angiogram and MT WITHOUT brain CT, if LVO is clinically suspected during CC .

Methods: A policy of direct MT without brain CT in LVO during CC was adopted at our center in $1 / 2016$. From $1 / 2016$ to $6 / 2017,3$ patients underwent direct MT. We included one comparative case prior to this time period in which a CT brain was done prior to MT. In cases of direct MT, the same access sheath placed by cardiologists was used for MT and the same single plane cardiac angiography suite was utilized for MT without moving the patient. We measured the feasibility of this approach for MT, accuracy of suspected LVO diagnosis, safety and functional outcomes.

Results: In the 3 patients undergoing direct MT without brain CT, the mean age was $68+/-2$ and all were females. The one patient who underwent indirect MT (after obtaining CT brain) for LVO during CC was 78 years old and male. In all 3 direct MT cases, it was feasible to perform a successful MT in the same cardiac catheterization laboratory in single plane. LVO was seen in all 3 patients on the cerebral angiogram prior to MT. The median time to first cerebral angiogram and time to revascularization was 17 (IQR 16-21.5) and 46 (IQR 38.5-65.5) minutes respectively in the three direct cases and 48 and 164 minutes respectively in the indirect case. Successful revascularization was achieved in all the case (TICI 2b-3).

Conclusions: Direct MT without prior brain imaging may save critical time to reperfusion with comparable safety in suspected LVO stroke during CC.

Keywords: Acute Ischemic Stroke Intervention, Angiographic Technology, Atherosclerosis, Cerebrovascular Disease, Diagnostic Neuroradiology

Financial Disclosures: The authors had no disclosures.

Grant Support: None. 


\section{Poster 84}

\section{SCAR Status (Sex, Contralateral Occlusion, Age, and Restenosis) to Risk Stratify Patients Undergoing Carotid Artery Stenting}

Vasu Saini, $M D^{1}$, Joshua Lukas, $M D^{1}$, Priyank Khandelwal, $M D^{1}$, Dileep R Yavagal, $M D^{1}$, Amer M Malik, $M D^{1}$, Seemant Chaturvedi, $M D^{1}$

'University of Miami Miller School of Medicine, Miami, Florida, USA

Introduction: Several studies have compared the risk of carotid artery stenting (CAS) versus carotid endarterectomy (CEA). Long-term outcomes are well-established to be similar between both treatment groups. Few studies have examined the perioperative risk between the two procedures. With similar long-term outcomes, exploration of the criteria that might identify CAS to be beneficial to a subgroup of patients is warranted.

Methods: We selected patients from within our institution that underwent CAS following either internal or common carotid artery stenosis. From this group, we identified four factors which comprise the SCAR status (sex, contralateral carotid occlusion, age, and restenosis) with SCAR-negative being either female $<$ age 75 , contralateral carotid occlusion or restenosis. All others were labeled SCAR-positive. We calculated complication rates in each group of patients and compared with previous data as outlined in Touze et al. 2013.

Results: We analyzed the predictive value of the SCAR status in 61 patients who underwent CAS at our institution from 2010 to 2014. Of our 61 patients, 48 were SCAR-positive (79\%) and 13 were SCAR-negative (21\%). The mean age is $67+8$. There were 7 perioperative complications, which were defined as stent re-occlusion or decline in neurological function. Six of the 7 complications were in the SCAR- positive group and 1 was in the SCAR-negative group. All had multiple chronic vascular risk factors and 3 had prior cardiovascular events. Five patients had a lesion length greater than $1 \mathrm{~cm}$. Two of the 7 had proximal protection devices during the procedure while 5/7 had distal protection devices. The SCAR-negative CAS group had a complication rate of $7.7 \%(1 / 13)$ versus $12.5 \%(6 / 48)$ in SCAR-positive group, though the small sample size limits accurate comparison to the absolute risk of CEA identified at 5.6\% in Touze et al. 2013.

Conclusions: We have shown the SCAR status to have predictive value in our patient population who underwent CAS. We identified patients who were SCAR-negative to have fewer perioperative complications than the SCAR-positive group. This scoring system can help to identify patients at increased risk of perioperative stroke or death and aid in identifying which population will benefit from CAS.

Keywords: Acute Ischemic Stroke Intervention, Atherosclerosis, Carotid Stenting And Angioplasty, Endovascular Therapy, Stenting

Financial Disclosures: The authors had no disclosures.

Grant Support: None. 


\section{Poster 85}

\section{A Predictive Model to Measure Treatment Outcomes in a Stroke Belt State}

Matthew C Scalise, $B S^{1}$, Benjamin Bailes, $B S^{1}$, Leanne Brechtel, BS ${ }^{1}$, Zachary Conn, $B S^{1}$, Jordan Gainey, BS ${ }^{1}$, Thomas Nathaniel, $P h D^{1}$

${ }^{1}$ USC School of Medicine-Greenville, Greenville, South Carolina, USA

Introduction: Major contributors to stroke mortality in the stroke belt include a high prevalence of hypertension, diabetes, heart disease, and obesity. However, it is not clear whether existing treatment efforts resulted in improved functional outcome in stroke patients within stroke belt states. This issue was investigated in this study.

Methods: A retrospective data analysis of 4,665 stroke patients was analyzed. Binary logistic regression was used to examine clinical factors associated with improved functional outcome in acute ischemic stroke patients eligible to receive rtPA. Predictor variables for each logistic regression model were selected by stepwise regression and variables with $\mathrm{p}<0.01$ remained in the model. Predictive power of each regression model was analyzed using ROC analysis. Multicollinearity was examined using variance inflation factors to confirm no significant interactions among independent variables existed.

Results: In rtPA treated acute ischemic stroke patients, only aphasia at presentation was positively associated with functional ambulation at discharge $(\mathrm{OR}=2.270, \mathrm{P}=0.002)$. Conversely, increasing age $(\mathrm{OR}=0.942, \mathrm{P}=$ $<0.001)$, congestive heart failure $(\mathrm{OR}=0.496, \mathrm{P}=0.040)$, NIH stroke scale score [NIHSS] $(\mathrm{OR}=0.876, \mathrm{P}=0.001)$, and antihypertensive medication $(\mathrm{OR}=0.436, \mathrm{P}=0.023)$ were negatively associated with functional ambulation. In non-rtPA treated patients, increasing age $(\mathrm{OR}=0.961, \mathrm{P}=<0.001)$, female gender $(\mathrm{OR}=0.686, \mathrm{P}=0.028)$, previous stroke $(\mathrm{OR}=0.538, \mathrm{P}=$ $0.002)$, NIHSS ( $\mathrm{OR}=0.899, \mathrm{P}=0.005)$ and atrial fibrillation $(\mathrm{OR}=2.133, \mathrm{P}=$ $0.001)$ were associated with worse functional ambulation at discharge.

Conclusions: Due to the higher risk of stroke, better understanding of clinical characteristics from a state within the stroke belt could provide information needed to address and decrease the burden of stroke. Analysis of clinical-level population data on ischemic stroke can aid our understanding of how clinical characteristics influence treatment decisions.

Keywords: Acute Ischemic Stroke Intervention, Thrombolytics, TPA

Financial Disclosures: The authors had no disclosures.

Grant Support: None. 


\section{Poster 86}

\section{Does Administration of Intravenous Contrast Impact Outcomes Upon Large Vessel Occlusion Patients Undergoing Intra-Arterial Therapy?}

Kavit Shah, $M D^{1}$, Stephan A Mayer, MD, $F C C M^{1}$, Maximilian K Kole, $M D^{1}$, Horia L Marin, $M D^{1}$, Lonni Schultz, PhD' ${ }^{1}$, Daniel J Miller, $M D^{1}$

${ }^{1}$ Henry Ford Hospital, Detroit, Michigan, USA

Introduction: Recent guidelines published by the American Heart Association/American Stroke Association strongly recommend urgent noninvasive vascular imaging in patients eligible for intra-arterial therapy (IAT) who present with a suspected large vessel occlusion (LVO) 1. CT Angiography (CTA) is typically the most expedient test; however, it requires administration of intravenous (IV) contrast. Additional contrast is administered if the patient undergoes digital subtraction angiography (DSA) for IAT. This has raised concerns about possible development of acute kidney injury (AKI) and/or contrast induced nephropathy (CIN)2-4. We sought to investigate what impact IV contrast had upon renal function and resultant outcomes of LVO patients undergoing IAT.

Methods: A retrospective chart review was conducted upon 130 consecutive patients who underwent IAT for LVO from 1/2014-8/2017. Primary outcomes included discharge and 90-day modified rankin scales (mRS). Patients were grouped into favorable $(\mathrm{mRS} \leq 2)$ and unfavorable $(\mathrm{mRS}>3)$. AKI was defined as an increase in serum creatinine ( $\mathrm{SCr}$ ) $\geq 0.3$ within 48 hours while CIN was defined as an increase in SCr $\geq 0.5$ or $25 \%$ at $48-72$ hours5.

Results: Of 130 patients (mean age $67.1 \pm 16$ ), 59 (45\%) were males and 71 (55\%) were females. At discharge, 30 (23\%) had a favorable mRS. Of the 96 patients with 90 -day follow-up available, $31(32 \%)$ had a favorable mRS. A total of 12 patients (9\%) suffered AKI while 4 patients (3\%) suffered CIN during their admission; however, there was no significant difference in discharge outcomes ( $\mathrm{p}=0.203$ and 0.791 , respectively). Of the 96 patients with 90-day mRS, a total of 8 (8\%) suffered AKI while $2(2 \%)$ suffered CIN. Again, there was no significant difference in outcomes $(\mathrm{p}=0.211$ and 0.343 , respectively).

Conclusions: IAT is associated with AKI and/or CIN in a small percentage of our patients; however, there was no significant impact upon outcome.

Keywords: Acute Ischemic Stroke Intervention, Angiogram, Endovascular Therapy, Intra-Arterial Therapy, Stroke

Financial Disclosures: The authors had no disclosures.

Grant Support: None. 
Poster 87

\title{
Fine Structure of Cerebral Thrombi Retrieved by Mechanical Thrombectomy Devices in Ischemic Stroke Patients
}

\author{
Meghana Shownkeen ${ }^{1}$, Karen Bates, PhD ${ }^{1}$, Patricia Blackwelder, PhD ${ }^{1}$, Dileep $R$ \\ Yavagal, $M D^{1}$ \\ ${ }^{1}$ University of Miami, Coral Gables, Florida, USA
}

Introduction: In order to improve stroke mechanical thrombectomy, a better understanding of the fibrin morphology of stroke thrombi and their association with etiology, first-pass success and clinical outcomes is needed. Scanning electron microscopy (SEM) can show fixed thrombi at low (100x) and high magnifications $(>20,000 \mathrm{x})$ for analysis of the relationships between cell and fiber morphology. We hypothesize that fine structure of the thrombi correlates with stroke etiology, first-pass success, and clinical outcome.

Methods: We used field emission scanning electron microscopy (SEM) to describe the fine structure of stroke-causing thrombi. Thrombi were collected from 15 acute ischemic stroke patients who underwent MT and consented to tissue collection from March 2017 to August 2017. Thrombi were fixed, prepared, and imaged in a Phillips XL-30 Field Emission SEM. Image J was employed to quantify observed fiber and cell relationships.

Results: Retrieved thrombi were from patients with atrial fibrillation, carotid, and cryptogenic etiology. There was a consistent pattern of fibrin formation and cell type distribution across all etiologies. We observed aggregation of fibers on the outside of the clots with more individual fibers and diverse cell types in the interior. Thrombi were red blood cell dominant, followed by white blood cells and platelets. Interestingly, thrombi presenting with exterior aggregated fibrin exhibited isolated surface platelets. Imaging data indicates that fibers coalesce to form a crust surrounding the thrombi and may indicate a mature stage of formation. Additional ongoing data collection examines these trends as related to individual history and etiology of thrombi. Statistical results will be presented.

Conclusions: SEM analysis of thrombi from stroke MT identifies distinct stages of fibrin formation in thrombi, which may prove to be important in improving clinical management of acute stroke. Also, further characterization of distinct formational stages of thrombi may help with developed new interventional tools and therapeutics.

Keywords: Acute Stroke, Ischemic Stroke, Thrombosis, Mechanical Thrombectomy, Basic Sciences

Financial Disclosures: The authors had no disclosures.

Grant Support: None. 


\section{Poster 88}

\section{Safety and Efficacy of Eptifibitide with Dichotomous Dosing Protocol in Conjunction with Hyperacute Neuroendovascular Intervention}

Ashik Shrestha, $M D^{1}$, Phillip Ye, $B A^{2}$, Danielle Crotty, PharmD ${ }^{1}$, Ting Zhou, $M D^{1}$, Karthikeyan Arcot, $M D^{2}$, David Turkel-Parrella, $M D^{2}$, Ambooj Tiwari, $M D M^{2} H^{2}$, Jeffrey Farkas, $M D^{1}$

${ }^{1} \mathrm{NYU}$ Langone Hospital - Brooklyn, Brooklyn, New York, USA; ${ }^{2} \mathrm{NYU}$ Langone Hospital - Brooklyn

Interventional Neuro Associates, Brooklyn, New York, USA

Introduction: Eptifibitide is a commonly used antithrombotic shown to reduce ischemic complications related to percutaneous coronary intervention. Recent findings suggest that eptifibatide administration has the potential to improve post-procedural outcomes in the context of neuroendovascular therapy for acute ischemic stroke.

Methods: 49 patients between 2014 and 2017 underwent thrombectomy for acute stroke and received eptifibitide. Cumulative and average eptifibatide dosages were determined for all patients. Peri- and and post-procedural bleeding complications were categorized into: intercranial hemorrhage (ICH), symptomatic intercranial hemorrhage (sICH), and peripheral bleeding (retroperitoneal, access site bleeding, and GI bleeding). Additionally, reperfusion Thrombolysis in Cerebral Infarction (TICI) scores as well as discharge modified Rankin Scales (mRS) were also collected. Patients were divided into those who received an average infusion rate of $0.75 \mathrm{mcg} / \mathrm{kg} / \mathrm{min}$ or higher and those who received lower, with rates of functional and clinical outcomes analyzed. An identical analysis was done for patients above and below median cumulative dosage $(0.32 \mathrm{mg} / \mathrm{kg})$.

Results: Of 49 total patients, 16 (32.7\%) patients were found to have intracranial hemorrhage, with 5 showing resulting clinical symptoms. 14 patients (28.6\%) experienced peripheral bleeding complications. The mean eptifibatide infusion for the selected patients was $0.75 \mathrm{mcg} / \mathrm{kg} / \mathrm{min}$ with a median cumulative dosage of $0.32 \mathrm{mg} / \mathrm{kg}$. On analysis, patients who received a higher average infusion of eptifibitide had higher incidence of all-cause bleeding complications $(\mathrm{p}<0.05)$, however individual analysis of each bleeding complication showed no significant relationship ( $p>0.05$ ). Additionally, patients who received higher infusions of eptifibitide or higher cumulative doses of eptifibitide increased rate of achieving TICI scores of $2 c-3$ in comparison to patients who achieved a TICI score of $2 b(p<0.05)$.

Conclusions: Usage of eptifibatide for patients undergoing neuroendovascular therapy for acute stroke at increased dosages may increase risk of overall bleeding complications. Higher dosage or infusion rate of eptifibatide may contribute to better post-procedural cerebral reperfusion.

Keywords: Acute Ischemic Stroke Intervention, Antiplatelet, Drugs Side Effects, ICH, TICI

Financial Disclosures: The authors had no disclosures.

Grant Support: None. 
Poster 89

\section{Workflow Metrics for MRI Selection of Patients for Endovascular Therapy in the GOLIATH Trial}

Claus Z Simonsen, $M D, P h D^{1}$, Albert J Yoo, $M D, P h D^{2}$, Sanja Karabegovic, $M D^{1}$, Lasse Speiser, $M D^{1}$, Mads Rasmussen, MD, OhD ${ }^{1}$, Leif H Sørensen, $M D^{1}$

${ }^{1}$ Aarhus University Hospital, Aarhus, Denmark; ${ }^{2}$ Texas Stroke Institute, Dallas, Texas, USA

Introduction: MRI has been criticized for being too time consuming for the evaluation of hyperacute stroke patients eligible for reperfusion therapy. Using data from the GOLIATH randomized trial, we aimed to study the workflow of patients undergoing MRI before endovascular therapy (EVT) and compare the time measures with CT-based studies.

Methods: GOLIATH randomized 128 patients with anterior circulation large vessel occlusion stroke within six hours of onset to EVT using either general anesthesia (GA) or conscious sedation (CS) (1:1 allocation). We compared standard workflow time measures to ESCAPE and SWIFT PRIME, two CT-based studies that emphasized rapid workflow.

Results: There was no difference in symptom onset to admission. Time used in GOLIATH was 101 minutes, interquatile range (IQR) 63.5-197. In ESCAPE it was 107.5 minutes (IQR 49.5-224) and in SWFT-PRIME 109.5 minutes (IQR 54-192.5.) In GOLIATH, door-to-scanner interval was a median of 9 minutes (IQR 6-13), versus 19 (IQR 11-29) and 16 minutes (IQR 10-23.5) in ESCAPE and SWIFT-PRIME respectively. Median time from the beginning of scan to groin puncture was 56.5 minutes (IQR 44.573.5) in GOLIATH and 51 minutes (IQR 39-68) in ESCAPE (not reported in SWIFT-PRIME). The median time from admission to groin puncture was 68 minutes (IQR 54.5-87) in GOLIATH and 90 minutes (IQR 69-120) in SWIFT-PRIME (not reported in ESCAPE.)

Conclusions: MRI can be performed with no significant difference in time delay compared to $\mathrm{CT}$ when evaluating stroke patients with large vessel occlusion for EVT.

Keywords: Acute Ischemic Stroke Intervention, Imaging, MRI, Endovascular Therapy

Financial Disclosures: The authors had no disclosures.

Grant Support: None. 


\section{Poster 90}

\section{Role of Flat Panel Cone Beam Computed Tomography in Detecting ICH}

Amrinder Singh, M.D. ', Ashish Kulhari', Siddhart Mehta' ${ }^{1}$, Briana DeCarvalho', Anna Barminova', Hemal Patel', Spozhmy Panezai' , Jawad Kirmani, MD'

1 JFK Stroke and Neurovascular Center, Seton Hall Hackensack Meridian School of Medicine, Edison, New Jersey, USA

Introduction: Intracranial hemorrhage is the second most common stroke and leaving many of its survivors with persisting neurological deficits. Endovascular intervention carries the peri-procedural adverse risk of hemorrhage in treatment of both cerebral aneurysm and acute ischemic stroke. A new C-arm system with 3D functionality extends the capability of C-arm imaging to include soft-tissue applications by facilitating the detection of low-contrast objects-Cone beam CT (CBCT). Our goal was to evaluate the application of this technology in detection of intracranial hemorrhage in patients undergoing neurointervention.

Methods: Between 2011-2016, CBCT was performed in 8 patients during neurointervention procedures at our university affiliated community based comprehensive stroke center, for early detection of Intracerebral Hemorrhage, due to suspicion of peri-procedural perforation complication.

Results: Of a total 221 stroke patient that received acute stroke intervention, a total of 8 patients received CBCT who underwent neurointervention. Six of 8 patients $(75 \%)$ patients presented with acute ischemic stroke, 1 with cerebral aneurysm and 1 with SAH. Mean age was 73 years (Range 49-93); $62.5 \%(5 / 8)$ were females. With the use of CBCT intra-operatively, Left basal ganglia hemorrhage, IVH with midline shift was detected in patient who underwent L ICA stenting and Left M1 mechanical thrombectomy. SAH patient's CBCT revealed worsening diffuse SAH with hydrocephalus. All patients had thin sliced CT head prior and after procedure for comparison of ICH with CBCT. These findings were confirmed on Thin sliced CT. No ICH/ IVH was noticed on rest of the patients. Volume of ICH was not calculated on CBCT.

Conclusions: We found that implementation of CBCT was successful in detecting intracranial hematomas. Our study was limited due to small population. Given the opportunity for expediting decision making in this critical setting, the use of intra-operative CBCT for inclusion or exclusion of hemorrhage warrants further large population based prospective trial. * Due to insufficient space for supporting authors, we had to include author names in Title*

Keywords: Acute Stroke, Hemorrhage

Financial Disclosures: The authors had no disclosures.

Grant Support: None. 


\title{
Poster 91
}

\section{Mechanical Thrombectomy in Elderly Stroke Patients with Mild-to-Moderate Baseline Disability}

\author{
Diana Slawski, BS ${ }^{1}$, Hisham Salahuddin, $M D^{2}$, Julie Shawver, PA-C', Syed Zaidi, \\ $M D^{4}$, Mouhammad Jumaa, $M D^{4}$ \\ ${ }^{1}$ The University of Toledo College of Medicine, Toledo, Ohio, USA; ${ }^{2}$ University \\ of Texas Southwestern Medical Center, Dallas, Texas, USA; ${ }^{3}$ ProMedica Toledo \\ Hospital, Toledo, Ohio, USA; ${ }^{4}$ The University of Toledo Medical Center, Toledo, \\ Ohio, USA
}

Introduction: The number of elderly patients suffering from ischemic stroke is rising. Randomized trials for mechanical thrombectomy (MT) generally excluded patients over age 80 with baseline disability. The aim of this study is to understand the efficacy and safety of MT in elderly patients, many of whom may have baseline impairment.

Methods: Between Jan 2015 and April 2017, 96 consecutive patients $\geq 80$ years old who underwent MT for stroke were selected for chart review. Data included baseline characteristics, time to treatment, rate of revascularization, procedural complications, mortality, and 90-day good outcome defined as modified Rankin scale (mRS) score $0-2$ or return to baseline.

Results: Out of 96 patients, 50 had mild baseline disability (mRS $0-1$ ) and 46 had moderate disability (mRS 2-4). Good recanalization was achieved in $84 \%$ of patients, and rate of symptomatic hemorrhage was $6 \%$. At 90 days, $34 \%$ of patients achieved good outcome. There were no significant differences for good outcome in those with mild or moderate baseline disability ( $43 \%$ vs. $24 \%, p=0.08)$, age $\leq 85$ or $>85$ years $(40.8 \%$ vs $26.1 \%, p=0.19)$ and treatment within or beyond 8 hours $(39 \%$ vs $20 \%, \mathrm{p}=0.1)$. Mortality was $38.5 \%$ at 90 days, and was significantly higher in patients with moderate compared to mild disability $(52.2 \%$ vs $26 \%, \mathrm{p}=0.01)$. ASPECTS score and NIHSS predicted good outcome regardless of baseline disability $(\mathrm{p}<.001$ and $\mathrm{p}=0.009$, respectively).

Conclusions: Advanced age, baseline disability, and delayed treatment are associated with suboptimal outcomes after MT. Redefining good outcome to include return to baseline functioning demonstrates that overall, one-third of elderly patients with baseline mRS 0-4 benefit from MT. This suggests reallife utility of this treatment.

Keywords: Acute Ischemic Stroke Intervention, Mechanical Thrombectomy, Acute Stroke

Financial Disclosures: The authors had no disclosures.

Grant Support: None. 


\section{Poster 92}

\section{Radiographic and Clinical Variables Correlating with Outcome After Endovascular Thrombectomy in Basilar Artery Occlusion (BAO)}

Jazba H Soomro, MD' ${ }^{1}$, Amrou Sarraj, Associate Professor', Liang Zhu, Statistician ${ }^{2}$

'University of Texas Health Sciences at Houston, Houston, Texas, USA;

${ }^{2}$ University of Texas Health Sciences, Houston, Texas, USA

Introduction: Early recanalization remains the main factor that can improve outcomes in BAO. We sought to evaluate the correlation between clinical and radiographic variables with favorable outcomes after endovascular thrombectomy (EVT) in BAO. .

Methods: Retrospective cohort from single comprehensive center of AIS patients with BAO from June 2015 to June 2017. PC-ASPECT score used to evaluate extent of hypodensities in posterior circulation. CTA collateral score (CS) used to evaluate collateral flow, 0 , no posterior communicating artery; 1 , unilateral; 2, bilateral. MRI DWI- ASPECT used for extent of infarcts post EVT. Primary outcome was good mRS of $0-2$ at 3 months. The correlation between NIHSS and PC-CT ASPECT, clot location, door to groin puncture (GP) and final infarct were evaluated. Correlation between CS, final infarct and good outcome at 90 days were evaluated.

Results: 18 patients who met the criteria were included. Mean age 71 (3484), baseline NIHSS 17 (5-35), pc-ASPECT 7 (5-10). CS 2 present in 16 patients (88.89\%), clot location; mid 6 (33\%), distal 9 (50\%), entire length 3 (16\%). Baseline NIHSS was not significantly correlated with pc-CT ASPECT, location of the clot, door to GP, and time to recanalization. Negative correlation was found between NIHSS and DWI-ASPECT $(r=-0.62 \mathrm{p}<0.05)$. Good outcome was present in $6(46 \%)$. The presence of good CS was not significantly correlated with good outcome at 90 days. There was a significant correlation between DWI ASPECT post EVT and good outcome at 90 days ( $\mathrm{r}=$ $-0.81 \mathrm{p} 0.03$ ).

Conclusions: Our results are limited by small sample size showed no correlation between CS and good outcome. Baseline NIHSS to be a predictor of DWI- ASPECT post EVT, which correlated with good outcome at 90 days.

Keywords: Ischemic Stroke, Acute Stroke, Basilar, Aspects, Acute Ischemic Stroke Intervention

Financial Disclosures: The authors had no disclosures.

Grant Support: None. 
Poster 93

\section{Pediatric Stroke: Neuroimaging of Rare Cerebral Arteriopathies and Vascular Abnormalities}

Navi Nallasamy, $M B B S^{1}$, Priya Sundaram-Simonelli, $M D^{1}$

${ }^{1}$ Cleveland Clinic, Cleveland, Ohio, USA

Introduction: This exhibit presents the application of various state-ofthe-art neuroradiological modalities in the assessment of cerebral arteriopathy associated with pediatric stroke. The objective is to give an overview of the prevalence, presentation, work up, and treatment of pediatric stroke with emphasis on cerebral arteriopathy using few rare cases. A review of common and uncommon cases is discussed along with their radiological presentation.

Methods: We present a series of cases demonstrating imaging findings of cerebral arteriopathies and vascular abnormalities using CT, CTA, MRI, and MRA (T1/T2/DWI). We discuss the etiology, classification, anatomic, and pathophysiology of cases which include: (1) Fibromuscular dysplasia (2) Hereditary hemorrhagic telangiectasia (3) PHACES syndrome (4) Moyamoya disease (5) Primary angiitis of CNS (6) Intracranial dissection, and (7) Secondary vasculitis.

Results: Key clinical and imaging findings are discussed for diagnoses and its impact on patient management. Pathologic features will be presented as it correlates to the diagnostic imaging features.

Conclusions: Stroke is infrequent in the pediatric age group but has high morbidity and mortality. For pediatric stroke, MRI is the first line imaging modality. However, it may not be readily available in the emergency setting. $\mathrm{CT}$ is less sensitive but readily available in such cases. Angiography is gold standard and is often necessary in suspected small and distal vessel pathologies. Arteriopathy is the cause of up to two-thirds of pediatric stroke, most often resulting in ischemic stroke. Delayed work-up and diagnosis may affect treatment and prognosis. Early identification of underlying disease process with the help of neuroimaging expedites the management strategies.

Keywords: Cns Vasculitis, Diagnostic Neuroradiology, Stroke, MRI, Vascular Imaging

Financial Disclosures: The authors had no disclosures.

Grant Support: None. 


\section{Poster 94}

\section{Endovascular Repair of the Ruptured Basilar Artery Bifurcation Aneurysms Including Those with Poor Grades}

Yahia M Lodi, MD, FAHA, FANA, FAAN', Varun V Reddy, $M D^{2}$, Shamaelah Javed, $M D^{3}$, Waleed Elnour, $M D^{4}$, Sumeet Multani, MD ${ }^{4}$, Tony Wong, MD, Ph. $D^{5}$, Scott Brehaut, $M D^{6}$

${ }^{1}$ Upstate Medical University, Binghamton/UHS-WMC/MVHS-Health, Binghamton, New York, USA; ${ }^{2}$ UHS-WMC/Upstate Medical University, Binghamton/MVHS-Health, Johnson City, New York, USA; ${ }^{3}$ UHS-Wilson Medical Center, Johnson City, New York, USA; ${ }^{4}$ UHS-WMC/Upstate Medical University, Binghamton, Johnson City, New York, USA; ${ }^{5}$ UHS-WMC/Upstate Medical University/MVHS-Health, Utica, New York, USA; ${ }^{6}$ MVHS-Health, Utica, New York, USA

Introduction: Endovascular repair of the basilar artery bifurcation aneurysms (BABA) remained the preferred approach, as clipping is associated with a higher chance of mortality and morbidity. Additionally, surgical clipping is not offered to those with poor grades. Objectives: To evaluate the outcome of patients with ruptured BABA who underwent endovascular repair including those with poor grades.

Methods: Consecutive patients who underwent envovascular repair of ruptured BARA aneurysm from January 2011 to December 2016 were retrospectively analyzed. Patients long-term outcome was measure using modified Rankin Scale (mRS)

Results: Results: 24 patients with median age 54 (31-71), 19 women, median aneurysm size $11 \mathrm{~mm}(4-30)$ and 14 wide-neck underwent repair; primary coiling 15, stent-assisted coiling 9 and 13 required ventriculostomy. Presenting H\&H grades; I in 7, II in 6, III in 4 and IV in 7. Pontine stroke developed in one who achieved mRS 4 and ventriculostomy related subdural hemorrhage in one (initial grade was IV, no improvement and died). Immediate complete and near complete aneurysm obliteration in 79\% (19; complete 10 , near complete 9 ) and subtotal in $21 \%$ (5). 90 days good outcome was observed in 75\% (mRS 0 in 14, mRS 1 in 2, mRS 2 in 2 ) and poor outcome in $25 \%$ (mRS 3 in 3, mRS 4 in 3, mRS 6 in 1). 7 patients (29\%) with $\mathrm{H} \& \mathrm{H} \mathrm{IV}$; mRS 0 in 2, mRS 1 in 2, mRS 4 in 2 and mRS 6 in 1. Aneurysm racialized in 9 cases (5 large, 3 giant) including subtotal obliterated cases and were retreated ( 5 stent-coil, 4 coiling).

Conclusions: Endovascular therapy for ruptured BABA is associated with low perioperative risk and good outcome including those with poor grades or required acute stent-assisted repair. Therefore, endovascular therapy should be offered for all ruptured BABA aneurysm including those with poor grades. Further studies are required.

Keywords: Endovascular Therapy, Subarachnoid Hemorrhage, Aneurysm Embolization, Stent Assisted, Basilar

Financial Disclosures: The authors had no disclosures.

Grant Support: None. 
Poster 95

Improving Time to Groin Puncture for In-House Strokes

Adam Barron, $M D^{1}$, Jody Manners, $M D^{1}$, Namir Khandker, $M D^{1}$, Ashutosh $P$ Jadhav, $M D, P h D^{\prime}$

'University of Pittsburgh School of Medicine, Pittsburgh, Pennsylvania, USA

Introduction: Inpatient stroke is associated with high morbidity and mortality, which is likely due to multiple factors including delayed time to recognition, associated comorbidities, and initial care from non-stroke trained providers. Implementation of a formalized 'stroke code' system can improve rapid diagnosis and reduce time to thrombolysis and thrombectomy.

Methods: In-house stroke cases identified at a single tertiary care center before and after implementation of a protocol utilizing improved provider communication, rapid imaging, and an accepted tool to identify cases of large vessel occlusion (the rapid arterial occlusion scale; RACE). Data from the electronic medical record including time of stroke code call, examination, imaging and intervention were recorded, and relevant time points compared between groups (code call to examination, examination to imaging, imaging to intervention).

Results: Cases of suspected in-house stroke analyzed as follows using descriptive statistics: 137 in the pre-protocol group (2013-2016) and 40 in the post-protocol group (2016-2017). Identification of all stroke (ischemic, hemorrhagic) increased by $22.3 \%$ after protocol initiation $(39.7 \%$ vs. $62.0 \%)$. Mean time to thrombolysis decreased by 10.2 minutes ( 38.7 vs. $28.5 \mathrm{~min}$ ) and the mean time to thrombectomy decreased by 24.2 minutes ( 53.0 vs. 28.8 min) in the post-protocol group after imaging was completed

Conclusions: Implementation of a streamlined stroke code protocol using the RACE scale improved identification of inpatient strokes and lessened time to intravenous and intra-arterial intervention. Future improvements focused on reduction in time from last seen well to stroke code call may expand therapeutic interventions.

Keywords: Door To Groin Puncture, Acute Ischemic Stroke Intervention Financial Disclosures: The authors had no disclosures.

Grant Support: None. 


\section{Poster 96}

\section{Does Location Matter in Stroke Care? Specialized and Nonspecialized Stroke Care Units in Thrombolytic Therapy}

Brice Blum, Student ${ }^{1}$, Leanne Brechtel, Student ${ }^{1}$, Meagan Reynolds, Student ${ }^{1}$, Thomas I Nathaniel, Ph.D., FAHA ${ }^{1}$

'University of South Carolina School of Medicine Greenville, Greenville, South Carolina, USA

Introduction: The benefits of a specialized stroke care unit over a nonspecialized stroke care unit is well documented in the literature. However, there have been concerns that the benefits seen in the SSCU do not consider the clinical variables that account for the criteria for rtPA eligibility. This issue was investigated in this study.

Methods: Retrospective data were collected from a stroke registry of patients admitted for acute ischemic stroke between January 1, 2010 to June 30, 2016. Univariate analysis determined differences in exclusion criteria between the SSCU and NSSCU, while multivariable binary logistic regression adjusted for confounding variables.

Results: Of the 1,446 patients determined eligible for rtPA, $65.7 \%$ of the patients were admitted to SSCU. Overall, $41.3 \%$ of patients received rtPA. For patients excluded from receiving rtPA in the SSCU: age $(\mathrm{OR}=1.024, \mathrm{p}=$ $<0.001$ ), altered level of consciousness ( $\mathrm{OR}=1.551, \mathrm{p}=0.041)$, risk of mortality GWTG $(\mathrm{OR}=1.090, \mathrm{p}=0.012)$, previous stroke $(\mathrm{OR}=1.638, \mathrm{p}=0.007)$ and NIH stroke scale $(\mathrm{OR}=0.865, \mathrm{p}=<0.001)$ were associated with exclusion from rtPA. In the NSSCU stroke population: age $(\mathrm{OR}=1.026, \mathrm{p}=0.012)$, history of atrial fibrillation $(\mathrm{OR}=2.494, \mathrm{p}=0.004)$, diabetes $(\mathrm{OR}=2.377, \mathrm{p}=$ $0.047)$, history of previous stroke $(\mathrm{OR}=2.782, \mathrm{p}=<0.001)$, weakness $(\mathrm{OR}=$ $0.365, \mathrm{p}=0.006)$, aphasia/language disturbance $(\mathrm{OR}=0.303, \mathrm{p}=<0.001)$, and antiplatelet medication $(\mathrm{OR}=0.578, \mathrm{p}=0.041$ ) were significantly associated with exclusion from rtPA.

Conclusions: The results of this study reveal that the proportion of clinical factors for exclusion is higher in the NSSCU than the SSCU in stroke patients excluded from rtPA. More factors are associated with rtPA exclusion in stroke patients admitted into NSSCU.

Keywords: TPA, Ischemic Stroke, Acute Ischemic Stroke Intervention

Financial Disclosures: The authors had no disclosures.

Grant Support: None. 
Poster 97

\section{Shorter Door-in to Door-out Time is Associated with Higher Likelihood of Endovascular Therapy}

Shashvat M Desai, $M D^{1}$, Benjamin Morrow, $M S N, R N^{1}$, Christian Martin-Gill, MD

$M P H^{1}$, Francis $X$ Guyette, $M D M P H^{1}$, Tudor $G$ Jovin, $M D^{1}$, Ashutosh $P$ Jadhav, MD

$P h D^{1}$

${ }^{1}$ University of Pittsburgh Medical Center, Pittsburgh, Pennsylvania, USA

Introduction: Endovascular therapy leads to improved clinical outcomes in patients presenting with large vessel occlusions (LVO); however outcomes are typically worse in patients triaged to a inter-facility transfer. A further understanding of the sources and impact of transfer delays is essential to improve outcomes in this population.

Methods: Data were analyzed from consecutive acute ischemic stroke patients with proximal LVO transferred to our comprehensive stroke center for consideration of endovascular therapy. The following variables were studied: door-in-door-out time (DIDO), baseline NIHSS/mRS, initial CT ASPECT, site of LVO, treatment and clinical outcome.

Results: A total of 309 patients were transferred from 14 referral hospitals to our CSC (from January to December 2016). Sixty-one patients (20\%) had a proximal LVO in the anterior circulation $(n=53)$ or basilar artery $(n=8)$. Forty patients (66\%) underwent endovascular thrombectomy and 21 patients (34\%) did not undergo endovascular thrombectomy - due to clinical improvement (33\%), large core or poor ASPECTS (48\%), premorbid disability (5\%), hemorrhagic transformation (5\%) and time from symptom onset $>24$ hours (9\%). Median DIDO time in the endovascular thrombectomy group was significantly better than the median DIDO time in patients who did not get endovascular thrombectomy (98.5 minutes vs 185 minutes, $\mathrm{p}$ value $=0.001$ ). After 90 minutes of DIDO time, the likelihood of undergoing IA therapy decreases by $7 \%$ with every additional 10 minutes of DIDO time. In patients presenting with NIHSS of 6 or higher, good outcomes were more frequent in the IA group versus non-IA group ( $33 \%$ versus $7 \%, p=0.06)$. In multivariable regression analysis, younger age and lower baseline NIHSS scores predicted good outcome in the IA group.

Conclusions: Shorter DIDO time is associated with higher likelihood of receiving endovascular therapy. DIDO time should be used as a clinical performance metric for stroke referring hospitals.

Keywords: Acute Ischemic Stroke Intervention, Endovascular Therapy, Ischemic Stroke, Care, Door To Needle

Financial Disclosures: The authors had no disclosures.

Grant Support: None. 


\section{Poster 98}

\section{Mobile Stroke Unit Serves Disaster Relief for Hurricane Harvey}

James C Grotta, $M D^{\prime}$, Stephanie A Parker, $R N^{2}$

${ }^{1}$ Memorial Hermann Hospital, Houston, Texas, USA; ${ }^{2}$ UT McGovern Medical

School, Houston, Texas, USA

Introduction: On August 25, 2017, Hurricane Harvey slammed into the south Texas coastline with $130 \mathrm{mph}$ winds causing devastating damage at landfall in Rockport Texas leaving residents homeless, without electricity, water, or supplies. A small emergency clinic was opened in Rockport staffed by emergency physicians and nurses from surrounding communities. There was insufficient power to operate xray or CT. On September 3, an inquiry was sent from the clinic to the our hospital in Houston to see if the Mobile Stroke Unit (MSU), housed at that hospital, might be available to assist.

Methods: When in operation, a $750 \mathrm{~kW}$ generator powered by the MSU truck engine supplies power to the CT scanner and unit. Responding to the request from Rockport, the authors drove the MSU 3.5 hours to Rockport.

Results: The MSU and CT scanner were powered off the generator, using about 50 gallons of gas each day. The local CT technologist was trained on use of the Ceretom scanner via telecommunication feed to the lead MSU CT technologist in Houston. Meals consisted of donated pizza and protein bars. The second day, another 50 gallons of gas were brought to Rockport to keep the MSU and CT scanner operational. After two days, no patients required a CT scan.

Conclusions: While in this case the MSU turned out not to be needed, our experience suggests a use for the MSU that has not been recognized previously, i.e. as a mobile Emergency Department, with diagnostic (including CT) and treatment capability where they don't otherwise exist either due to lack of resources or natural disaster. While currently being deployed only for stroke patients, and tested largely in urban environments in developed countries, our experience suggests a much broader worldwide application of the "mobile" approach to delivering medical care.

Keywords: TPA, New Innovation, Ischemic And Hemorrhagic Stroke, Imaging

Financial Disclosures: consultant: Stryker Corp, Frazer Ltd Grant Support: Genentech

Grant Support: None. 
Poster 99

\section{The Economics of Market Entry for Thrombectomy-Capable Acute Care Facilities}

Susan J Heilman, rn, mpm ${ }^{1}$, Kristin Truesdell, $n / a^{1}$

${ }^{1}$ Corazon, Inc., Pittsburgh, Pennsylvania, USA

Introduction: Endovascular treatment for stroke has increased in the US based upon clinical trial data demonstrating efficacy and cost effectiveness. With the advent of the DAWN trial, the window for an interventional strategy may be expanded. Expansion of hospital services for inclusion of thrombectomy care requires an investment in personnel, capital equipment, and services. The costs and decisions associated with market entry are outlined to assist strategic decision-making and service implementation.

Methods: Costs associated with neurointerventional service development were assessed at 6 facilities considering facility design, personnel, capital equipment, operating and administrative costs. Thrombectomy costs were benchmarked against Medicare inpatient stroke DRGs using a primary procedure code of 39.74 .

Results: Average program development expenditures were $\$ 7,531,058+$ $\$ 3,537,634$. The most significant expenditures were in personnel costs associated with recruitment and employment of neurologic specialists ranging from $\$ 3,305,246+\$ 465,762$. Facility investments were $\$ 3,174,312+$ $\$ 3,071,872$ depending upon the presence of an existing interventional suite.

Conclusions: The number of thrombectomy-capable facilities is expected to increase as research confirms the benefit of interventional stroke strategies. Economic investment in the development of a program can be scaled depending upon the availability of pre-existing personnel and imaging suites. If space and personnel are available, market entry can be initiated with an investment of $\$ 1,153,940$. Operating costs are dependent upon retention of a highly trained care team composed of neurosurgical, interventional, and medical providers. Service initiation is dependent upon a detailed business planning process to ensure adequate identification of population needs, infrastructure, and service maintenance expenditures.

Keywords: Stroke, Mechanical Thrombectomy

Financial Disclosures: The authors had no disclosures.

Grant Support: None. 


\section{Poster 100}

\section{Field Destination Triage of Stroke Patients with RACE Scale Reduces First Contact to Recanalization Time}

Mouhammad Jumaa, $M D^{1}$, Diana Slawski, $B S^{2}$, Syed Zaidi, $M D^{1}$

${ }^{1}$ The University of Toledo Medical Center, Toledo, Ohio, USA; ${ }^{2}$ The University of

Toledo College of Medicine, Toledo, Ohio, USA

Introduction: The aim of this study is to determine whether pre-hospital identification of stroke patients with suspected large vessel occlusion (LVO) and bypass to an endovascular capable center (ECC) is associated with shorter 'first medical contact' (FMC) to treatment times and improved outcomes.

Methods: We conducted retrospective review of consecutive patients who underwent Mechanical Thrombectomy (MT) and were initially triaged by local county Emergency Medical Services (EMS) before and after implementation of a protocol based on the Rapid aRterial Occlusion Evaluation (RACE) score. Between June 2013-July 2015, patients were triaged to the closest stroke center and then transferred to an ECC for MT (SA group). After July 2015, all patients with RACE scale $>5$ were triaged directly to an ECC (RA group).

Results: A retrospective review identified 113 patients between June 2013 and December 2016 (SA = 56, RA = 57). The RA group had significantly shorter first medical contact (FMC) to recanalization time ( $\mathrm{RA}=130 \mathrm{vs}$. $\mathrm{SA}=220.5 \mathrm{~min}(\mathrm{p}<0.001)$ and higher rate of excellent clinical outcome $(43.9 \%$ vs. $21.4 \% \mathrm{p}=0.015)$. Median transfer time in the SA group was 99 minutes.

Conclusions: Direct field triage of suspected LVO patients with RACE scale is associated with faster FMC to recanalization time and improved clinical outcomes in patients who undergo MT. Further studies are necessary to understand the generalizability of this approach.

Keywords: Acute Ischemic Stroke Intervention, Endovascular Therapy

Financial Disclosures: The authors had no disclosures.

Grant Support: None. 


\title{
Poster 101
}

\section{Continued Nationwide Decline in Stroke Related In-Hospital Mortality in United States}

\author{
Rakesh Khatri, $M D^{1}$, Mohtashim Arbaab Qureshi, MD', Mohammad Rauf Afzal, \\ $M D^{1}$, Anantha Ramana Vellipuram, $M D^{1}$, Salvador Cruz-Flores, $M D^{1}$, Gustavo J \\ Rodriguez, $M D^{1}$, Alberto Maud, $M D^{1}$
}

${ }^{1}$ Texas Tech University Health Sciences Center, El Paso, Texas, USA

Introduction: Previously a downward trend in stroke related in-hospital mortality was noted from 1996 to 2006 reflecting advancements in acute stroke care. Given ongoing improvement in acute stroke care models and therapies, our study assessed nationwide patterns in in-hospital mortality related to acute stroke for next decade.

Methods: Patients with acute stroke were identified from the nationwide in-patient sample database for the years 2007 to 2014 using International Classification of Diseases, Ninth Revision procedure codes. Time trends in the proportion of stroke hospitalizations that resulted in death were assessed.

Results: Between 2007 and 2014, overall stroke hospitalizations increased from 1017414 in 2007 to 2008 to 1114960 in 2013 to 2014, whereas percentage stroke hospitalizations that resulted in death decreased from $8.98 \%$ in $2007-2008$ to $7.34 \%$ in $2013-2014$ ( $\mathrm{P}<0.0001)$. Unadjusted Odds of mortality decreased regardless of stroke type: ischemic stroke (IS) (OR 0.798, 95\% CI 0.762 to 0.835 ), subarachnoid hemorrhage (SAH) (OR $0.885,95 \% \mathrm{CI}$ 0.810 to 0.968 ), and intracerebral hemorrhage (ICH) (OR $0.865,95 \%$ CI 0.824 to 0.908$)$. In multivariate analysis these odds remained significant (IS: OR $0.813,95 \%$ CI 0.770 to 0.859 ), SAH: OR 0.680 , $95 \%$ CI 0.611 to 0.757 , ICH: OR $0.707,95 \%$ CI 0.664 to 0.754 ) after adjusting with age, gender, race, medical comorbidities, in-hospital complications, insurance status and APRDRG Severity scale).

Conclusions: There is continued decline in Nationwide In-Hospital Mortality among hospitalized acute stroke patients. This reflects ongoing improvement in stroke care models and advancement in therapies

Keywords: Ischemic Stroke, SAH, ICH, Treatment

Financial Disclosures: The authors had no disclosures.

Grant Support: None. 


\section{Poster 102}

\section{Neurointerventionalist Role in Triaging Stroke from the Field}

Ritesh Kaushal, $M D^{1}$, Patrick Flynn, EMS Chief', Ariol Labrada, MD', Monica MejiaAcosta, $M D^{3}$, Roberto Sanchez, $M D^{3}$, Ana Mederos, $C E O^{3}$, Julie Riley, Neuroscience Director ${ }^{1}$, Sarah Janvier, Neuroscience Manager ${ }^{1}$, Ali Malek, MD ${ }^{1}$, Nils MuellerKronast, $M D^{1}$

${ }^{1}$ Advanced Neuroscience Network, Tenet Florida, Hialeah, Florida, USA; ${ }^{2}$ Hialeah EMS, Hialeah, Florida, USA; ${ }^{3}$ Palmetto General Hospital, Hialeah, Florida, USA

Introduction: Management of acute stroke especially Large Vessel Occlusion (LVO) is extremely time sensitive. Results from the STRATIS registry showed a 5.5\% absolute decline in the likelihood of achieving modified Rankin Scale score 0 to 2 for every hour delay in treatment. As endovascular therapy becomes the primary management tool in LVOs, this decrease of clinical benefit over time warrants optimization of the system of care.

Methods: Our hospital system has been working in very close collaboration with the Hialeah EMS. Starting January 2017, we initiated a new model towards optimal triage of acute stroke patients. Hialeah EMS has been given one phone per fire truck that only has the phone number of the neurointerventionalist. On reaching suspected stroke patient in the field, the EMS evaluate for signs of LVO, anti-coagulation status, current or past atrial fibrillation. If any of these are positive, EMS directly calls the neurointerventionalist from the field. The neurointerventionalist does assessment over the phone and/or facetime. The neurointerventionalist then immediately activates the team and the ER is notified.

Results: Starting January to August 2017, with the new model, the neurointerventionalist received 165 calls from EMS in the field. The team was activated 89 times for suspected LVO. 71 of these resulted in endovascular treatment for LVO. Of the 18 "blank" runs, 9 were intracranial hemorrhage and 9 were secondary to altered mental status, seizure or otherwise without LVO on non-invasive angiography. The median door-to-groin for the $71 \mathrm{LVO}$ intervention was 42 mins. In 2016, prior to implementation of this model, our door-to-groin was 76 mins for 94 LVOs treated.

Conclusions: Direct triaging of LVOs by neurointerventionalist with EMS from field can significantly reduce the door-to-groin time and consequently lead to better outcomes.

Keywords: Stroke, Acute Ischemic Stroke Intervention, Clinical Investigations

Financial Disclosures: The authors had no disclosures.

Grant Support: None. 
Poster 103

\section{Emerging Considerations in Regional Networks of Care}

Stacey Lang, rnc, bsn, cnrn ${ }^{1}$

${ }^{1}$ Corazon, Inc., Pittsburgh, Pennsylvania, USA

Introduction: Recent Centers for Disease Control and Prevent data was reviewed relative to time to treatment averages by geographic area in the United States. Despite treatment advances, early recognition and access to care continue to negatively impact outcomes in stroke. In response, key program elements combined with novel approaches to enhance patient access were identified, when in place, to significantly improve time to treatment metrics.

Methods: Rapid clinical advances dramatically increased the pool of patients affected by stroke who may benefit from expanded treatment options. Timely symptom recognition, fragmented transport systems, and limited provider availability, continue to challenge programs nationally in connecting eligible patients to available treatment within recommended time frames. Results of the DAWN Trial, as well as the 2016 Society of Vascular and Interventional Neurology (SVIN) Consensus document, present ample evidence of the need for change nationally with respect to networks of care and timely access to interventions. The landscape across the U.S. is complicated by the lack of uniformity in legislative and state based regulations around pre-hospital transport. The onus is on health care organizations to approach care delivery on a regional level and requires an expanded approach to strategic and programmatic planning and implementation.

Results: The Joint Commission (TJC) is developing an additional level of certification for U.S. stroke programs due to evolving clinical recommendations, particularly in Large Vessel Occlusion strokes. It is anticipated that programs certified as "Thrombectomy Only" will expand availability of treatment beyond traditional tertiary or quaternary centers. A comprehensive regional approach to program development is essential as providers deliver care to this expanded patient pool. A unique strategic planning and implementation method proven effective in diverse national markets will be presented.

Conclusions: In conclusion, programs who have embraced this methodology enjoy long-term financial viability, enhanced clinical outcomes, improved time to treatment results, and exceptional patient satisfaction.

Keywords: Stroke

Financial Disclosures: The authors had no disclosures.

Grant Support: None. 


\section{Poster 104}

\section{Emergent CTA is Associated with Faster IV TPA Delivery}

Radoslav I Raychev, $M D^{1}$, Jana Sawuger, RN, $B S N^{1}$, Nirav Patel, $M D^{1}$, Nima

Ramezan, $M D^{1}$, Mike Thompson, $M D^{1}$, George Wang, $M D^{1}$, Tze Kong, $M D^{1}$, Vikas

Rao, $M D^{1}$, Chiedozie Nwagwu, $M D^{1}$, Tri Tong, $M D^{1}$

'Saddleback Memorial Medical Center, Laguna Hills, California, USA

Introduction: Early diagnosis of large vessel occlusion (LVO) may facilitate patient triage in ED. However, multimodal imaging may delay IV TPA administration.

Methods: In response to updated guidelines establishing thrombectomy as standard of care for LVO, emergent CTA was included as part of the standard ED imaging protocol for all patients who presented to our institution with suspected acute ischemic stroke (AIS). In this prospectively collected database of consecutive patients treated with IV TPA since January 1st 2014 - present, we examined the relationship between multiple baseline factors and door-to-needle time Analyzed variables included: Walk-in status, Prehospital notification, Onset to ED arrival (min), Door to CT (min), Emergent CTA performed in ED prior to TPA, Thrombectomy, NIHSS $>/=6$; Age $>/=$ 65.

Results: Total of 150 patients were included in the analysis. The average door-to-IV TPA start significantly improved over time from 1:10 min in 2014 to $46 \mathrm{~min}$ in 2017. Among all analyzed patients, $77 \%(116 / 150)$ were transported by EMS, $76 \%(114 / 150)$ had NIHS $\geq 6,79 \%$ (119/150) were older than 65 . The median door-to-CT was $22 \mathrm{~min}$ (11-101), and the median door-toIV TPA delivery was $42 \mathrm{~min}$ (15-162). Thrombectomy was performed in $25 \%(38 / 150)$, and emergent CTA prior to IV TPA was obtained in $61 \%$ $(91 / 150)$. None of the patients who had undergone CTA had renal complications. Among all factors included in the multivariate model, the most significant predictors of door-to-needle $<60$ min were: 1) Door-to-CT (OR 0.94; $\mathrm{p}=0.008), 2)$ Thrombectomy (OR 2.88, $\mathrm{p}=0.029)$, and 3) Emergent CTA in ED (OR 2.68; $\mathrm{p}=0.017)$.

Conclusions: Thrombectomy and pre-IV TPA emergent CTA are associated with faster door-to-needle time likely due more rapid diagnosis of LVO. Emergent CTA should be considered as a standard imaging evaluation of all patients with suspected AIS.

Keywords: Acute Stroke, Endovascular Therapy, Angiographic Ct, Door To Needle, Lytics

Financial Disclosures: The authors had no disclosures.

Grant Support: None. 
Poster 105

\title{
Identify Patients Who Face Acute Delivery to a Comprehensive Stroke Center by EMS: FACE2AD Scale
}

\author{
Hiroshi Yamagami, Chief' ', Yoshinori Okuno, Resident ${ }^{2}$, Hiroharu Kataoka, Chief ${ }^{3}$, \\ Hidemori Tokunaga, Director ${ }^{4}$, Makoto Kobayashi, Director ${ }^{5}$, Nobuyuki Sakai, \\ Director ${ }^{6}$, Jun C Takahashi, Director ${ }^{3}$, Kazunori Toyoda, Director ${ }^{7}$, Masafumi Ihara, \\ Director ${ }^{2}$, Kazuyuki Nagatsuka, Director ${ }^{2}$ \\ ${ }^{1}$ Division of Stroke Care Unit, National Cerebral and Cardiovascular Center, \\ Suita, Japan; ${ }^{2}$ Department of Neurology, National Cerebral and Cardiovascular \\ Center, Suita, Japan; ${ }^{3}$ Department of Neurosurgery, National Cerebral and \\ Cardiovascular Center, Suita, Japan; ${ }^{4}$ Department of Neurosurgery, Nara City \\ Hospital, Nara, Japan; ${ }^{5}$ Emergency \& Critical Care Medicine, Tajima Emergency \\ \& Critical Care Medical Center, Toyooka, Japan; ${ }^{6}$ Department of Neurosurgery, \\ Kobe City Medical Center General Hospital, Kobe, Japan; ${ }^{7}$ Department of \\ Cerebrovascular Medicine, National Cerebral and Cardiovascular Center, Suita, \\ Japan
}

Introduction: Patients with acute ischemic stroke due to large vessel occlusion (LVO) should be triaged to an endovascular-capable hospital by emergency medical service (EMS). We designed a prehospital LVO prediction scale, which is constructed with assessments by EMS.

Methods: In the derivation cohort, we retrospectively examined 1157 patients transferred to our hospital by EMS because of suspicion of stroke within 24 hours of onset. LVO was defined as occlusion of internal carotid artery, middle cerebral artery (M1 or M2 portion), or basilar artery. We extracted factors associated with acute stroke due to LVO based on assessment by EMS, and developed a prehospital scale identifying LVO (FACE2AD scale). Thereafter, we validated the accuracy of this scale in consecutive 502 patients who were transferred to 4 stroke centers, and compared with previously reported 4 scales.

Results: Acute stroke due to LVO was diagnosed in 149 of $1157(13 \%)$ in the derivation cohort. We developed FACE2AD scale using factors related with LVO: assigning one point each for facial palsy, arm weakness, impaired consciousness (cannot say his/her name), atrial fibrillation, and diastolic blood pressure $\leq 85 \mathrm{mmHg}$, and two points for conjugate eye deviation. In validation cohort, 86 of 502 patients (17\%) were acute stroke with LVO. Optimal cut point of FACE2AD $\geq 3$ showed $80 \%$ sensitivity, $70 \%$ specificity, $39 \%$ positive predictive value, $98 \%$ negative predictive value and area under the curve (AUC) of $0.84(0.81-0.86)$ for prediction of LVO. FACE2AD scale had higher accuracy of predict LVO than RACE, CPSSS, PASS, and FAST-ED (AUC of FACE2AD 0.84 as the reference; RACE 0.78, p < 0.01; CPSSS 0.75, $\mathrm{p}<0.01$; PASS 0.77, $\mathrm{p}<0.01$; and FAST-ED 0.78; $\mathrm{p}<0.01$ ).

Conclusions: FACE2AD scale is a simple and reliable tool to identify ischemic stroke patients with LVO by EMS.

Keywords: Ischemic Stroke, Acute Ischemic Stroke Intervention

Financial Disclosures: The authors had no disclosures. 
Grant Support: This study was supported in part by Intramural Research Fund for Cardiovascular Diseases of National Cerebral and Cardiovascular Center (H26-4-1) 


\title{
Poster 106
}

\section{A Novel Ruthenium (II) Complex Potentially Inhibits Agonists Induced Platelet Activation: Impact on Akt/JNK Signaling}

\author{
Thanasekaran Jayakumar, Postdoctoral Fellow', Joen-Rong Sheu, Professor', \\ Chi-Li Chung, Medical Doctor ${ }^{3}$ \\ 'Department of Pharmacology, Taipei Medical University, Taipei, Taiwan; \\ ${ }^{2}$ Taipei Medical University, Taipei, Taiwan; ${ }^{3}$ Taipei Medical University Hospital, \\ Taipei, Taiwan
}

Introduction: Ruthenium complexes are considered as potential alternatives for platinum compounds in cancer therapy, and have been proved as promising anticancer drugs with high efficacy and low side effects. Platelet activation plays major role in cancer metastasis and progression. Thus, this study explored the effect of newly synthesized ruthenium complex, TQ-5 on human platelet activation.

Methods: Sheu et al [1] and Lin et al [2] were followed for the preparation of human platelet suspensions. As previously described [1,2], platelet aggregation was measured by a Lumi-Aggregometer (Payton Associates, Scarborough, ON, Canada). For the measurement of ATP release, a $20 \mu \mathrm{L}$ of luciferin-luciferase mixture was added $1 \mathrm{~min}$ before adding collagen, and the relative amount of ATP release was compared to the solvent control.The relative $\mathrm{Ca} 2+$ mobilization was measured as described previously [3]. Western blot analysis used to detect MAPKs signaling.

Results: At 3-5 $\mu \mathrm{M}$, TQ-5 inhibited collagen-induced platelet aggregation in washed human platelets, but TQ-5 inhibited platelet aggregation only at a maximum concentration of 500 and $100 \mu \mathrm{M}$ against thrombin and U46619induced stimulation, respectively. TQ-5 inhibited collagen induced ATP release and calcium mobilization $([\mathrm{Ca} 2+] \mathrm{i})$ without inducing cell cytotoxicity. TQ-5 inhibited collagen induced phosphorylation of Akt and c-Jun $\mathrm{N}$-terminal kinase (JNK), but not effectively inhibits ERK1/2 and p38-MAPK in human platelets. Additionally, TQ-5 significantly prolonged the closure time in whole blood and increased the occlusion time of thrombotic platelet plug formation in mice.

Conclusions: The results of this study demonstrates, for the first time, that a newly synthesized ruthenium complex TQ-5 exhibits potent antiplatelet activity by obstructing ATP release and $[\mathrm{Ca} 2+] \mathrm{i}$, and reducing activation of Akt/JNK signals. Collectively, these data suggest that TQ-5 could be established as therapeutic agent that aids stop or treat thromboembolic disorders, as it is reputable to be more effective than a well-known antithrombotic aspirin.

Keywords: Antiplatelet, Cerebral Protection, Cerebrovascular Disease, Coagulation, Pathophysiology

Financial Disclosures: The authors had no disclosures. 
Grant Support: This work was supported by grants (MOST1032320-B-038-017, OST104-2622-B-038-003, and MOST 104-2320-B-038045-MY2) from the National Science Council of Taiwan, and the Yuan's General Hospital-Taipei Medical University (104-YGH-TMU-01-3). One of the author 


\section{Poster 107}

\section{Aspirin and Anticoagulant Usage on Outcomes After Ruptured Arteriovenous Malformation: A Nationwide Inpatient Sample Analysis}

Rakesh Khatri, $M D^{1}$, Mohtashim Arbaab Qureshi, $M D^{1}$, Mohammed Rauf Afzal, $M D^{1}$, Anantha Ramana Vellipuram, $M D^{1}$, Salvador Cruz-Flores, $M D^{1}$, Gustavo J Rodriguez, $M D^{1}$, Alberto Maud, $M D^{1}$

${ }^{1}$ Texas Tech University Health Sciences Center, El Paso, Texas, USA

Introduction: Recently in nationwide study, neither long-term aspirin nor anticoagulant use were associated with differential mortality or complication rates after aneurysmal subarachnoid hemorrhage however their impact on outcomes of ruptured arteriovenous malformation is unclear.

Methods: Data from the Nationwide Inpatient Sample (NIS; 2006-2014) were analyzed in patients with a primary diagnosis of SAH and/or ICH with a diagnosis of an arteriovenous malformation. These patients were divided in two groups: receiving long-term aspirin or anticoagulant use versus neither. Multivariable logistic regression was performed to calculate the adjusted odds of in-hospital mortality, a nonroutine discharge (any discharge other than to home), or a poor outcome (death, discharge to institutional care, tracheostomy, or gastrostomy) for patients with long-term aspirin or anticoagulant use. Multivariable linear regression was used to evaluate length of hospital stay. Covariates included patient demographics, comorbidities, APDRG severity scale, and treatment of AVM including embolization, surgical resection and radiosurgery

Results: Our study examined 11066 patients with ruptured AVM. Patients taking either aspirin $(2.5 \%, \mathrm{n}=273)$ or anticoagulation $(1.7 \%, \mathrm{n}=185)$ were significantly older and had a greater burden of comorbid disease (hypertension, hyperlipidemia, atrial fibrillation and renal disease, $\mathrm{p}<0.001$ ). Inpatient mortality $(10.9 \%$ either aspirin/anticoagulation vs $9.2 \%$ neither, $\mathrm{p}=0.63$ ) Length of stay $(9.3 \pm 8.7$ days vs $11.0 \pm 13.5$ days $p=0.11)$ were not significantly different. In multivariable logistic regression models for outcome, discharge disposition was better in ASA/Anticoagulation group (home discharge OR 2.36, CI $1.40-3.98, \mathrm{p}=0.001$ ) but inpatient mortality was not different (OR 0.79, CI 0.34-1.82, $\mathrm{p}=0.58$ ).

Conclusions: In this nationwide study, neither long-term aspirin nor anticoagulant use was associated with differential mortality after ruptured arteriovenous malformation. Aspirin/anticoagulant use was associated with favorable discharge to home.

Keywords: Antiplatelet, Avm Embolization, SAH, Ruptured

Financial Disclosures: The authors had no disclosures.

Grant Support: None. 


\section{Poster 108}

\section{Combination Therapy of Endovascular Embolization and Stereotactic Radiosurgery for Ruptured Inoperable Arteriovenous Malformations}

Michael I Nahhas, $M D^{1}$, Scott $Y$ Rahimi, $M D^{1}$, Nathaniel Todnem, $M D^{1}$, Christopher A Witt, Medical Student ${ }^{1}$, John R Vender, $M D^{1}$, Cargill H Alleyne, $M D^{1}$

${ }^{1}$ Augusta University at The Medical College of Georgia, Augusta, Georgia, USA

Introduction: The management of cerebral arteriovenous malformations (AVMs) in general remains controversial. Ruptured AVMs in particular are associated with an increased risk of morbidity and mortality and carry a rehemorrhage risk up to $18 \%$. Some centers may choose to be more aggressive in treating ruptured AVMs because of this increased risk in re-hemorrhage. In this case series, we present data from a retrospective analysis of patients from our center with high-grade non-operative ruptured AVMs treated with a combination of endovascular embolization and gamma knife radiosurgery.

Methods: A retrospective analysis was performed on all patients with ruptured AVMs presenting to our center between 2010-2015. All patients were Spetzler-Martin grade III-IV and deemed not to be surgical candidates. We evaluated for changes in Modified Rankin scale (mRS), re-hemorrhage rates, and changes in size of nidus as outcome measures.

Results: Of the 16 patients who met inclusion criteria, 5 were females and 11 were males with an age range of 10-70 years (average: 46.63 years). The time range from embolization to follow up was 17-90 months (average 45.4 $+/-21.43$ months) with one patient lost to follow up. The average dose of gamma knife was $19+/-1.24 \mathrm{~Gy}$. The average mRS was 3.31 at the time of presentation, 2.06 at the time of gamma knife, and 1.53 at the time of last follow up. Follow up imaging included MRI and angiography. 7 patients (58.33\%) with repeat imaging had a complete obliteration of the AVM. The remaining 5 patients showed reduction in nidus diameter and volume. There has been no incidence of re-hemorrhage within this cohort.

Conclusions: Combination therapy of endovascular embolization and gamma knife radiosurgery is effective in treating grade III-IV AVMs that are not felt to be surgical candidates. Our small series had $58.33 \%$ obliteration, no re-hemorrhages, and no worsening in mRS scores over time.

Keywords: Cerebral Arteriovenous Malformations, Avm Embolization, Intracerebral Hemorrhage, Treatment

Financial Disclosures: The authors had no disclosures.

Grant Support: None. 
Platform Session I, Friday, November 10, 3:30-3:40 pm

\section{Thrombectomy Benefit: 6-24 Hour Time Window by Mode of Presentation: Secondary Analysis of the DAWN Trial}

Ashutosh Jadhav, $M D^{1}$, Tudor Jovin, $M D^{2}$

'UPMC, Pittsburg, Pennsylvania, USA

Introduction: Results of the DAWN trial recently demonstrated benefit of stent retriever thrombectomy over medical management in patients presenting with proximal anterior circulation large vessel occlusion in the 6-24 hour time window. Previous studies have suggested that wake up strokes (WUS) may have higher treatment effect after thrombectomy as compared to witnessed onset (WO) or unwitnessed onset (UWO) patients.

Methods: Data were analyzed from the DAWN study - a multicenter, prospective, randomized, clinical trial of patients presenting with LVO undergoing neurothrombectomy with the Trevo device versus medical management. A total of 206 patients were enrolled during the study. Univariate and multivariable logistic regression was used to assess relationship between outcome and mode of presentation.

Results: Of the 206 patients analyzed, mode of presentation in the DAWN trial was follows: WUS $(55.3 \%, \mathrm{n}=114)$, WO $(12.1 \%, \mathrm{n}=25)$ and UWO $(32.5 \%, \mathrm{n}=67)$ with baseline NIHSS $(17.2 \pm 4.7,14.7 \pm 4.0,19.3 \pm 6.0)$, MCA site of occlusion $(77.2 \%, 88.0 \%, 83.6 \%)$, TSLW to randomization $(13.4 \pm 3.7$, $10.0 \pm 3.7,14.1 \pm 4.9)$, baseline infarct volume $(12.4 \pm 11.7,8.2 \pm 8.8,11.3 \pm$ 10.9 ) and rate of successful recanalization mTICI $>2 \mathrm{~b}(81.8 \%, 81.8 \%, 89.7 \%)$. Nominal rates of good outcome $(33.3 \%, 40.0 \%, 25.4 \%)$, PH-2 $(2.1 \%$, $0.0 \%, 6.0 \%)$, sICH $(10.4 \%$ vs $0.0 \%, 1.8 \%)$ and 24 hour infarct volume were not different when comparing WUS vs WO and UWO. Treatment benefit (90 day mRS) was maintained across all 3 subgroups (WUS- $49.3 \% \mathrm{vs} 10.6 \%$, WO-63.6 vs 21.4 , UW-41.4\% vs $13.2 \%$; $\mathrm{p}^{*}$ interaction $=0.21$ ). Multivariate analysis identified age, NIHSS, ASPECTS, and diabetes as predictors of good outcome whereas the mode of presentation was not a predictor of good outcome.

Conclusions: Benefit of the endovascular therapy in appropriately selecting patients presenting in the late time window persists irrespective of mode of presentation.

Keywords: Acute Ischemic Stroke Intervention, Endovascular Therapy, Trevo, Angiographic Ct Perfusion, Door To Groin Puncture

Financial Disclosures: The authors had no disclosures.

Grant Support: None. 
Platform Session I, Friday, November 10, 3:40-3:50 pm

\section{Feasibility of RNA Extraction for RNA Sequencing Analysis in Acute Large Vessel Ischemic Stroke Thrombi}

Kunakorn Atchaneeyasakul, $M D^{1}$, Karen Bates, $P h D^{2}$, Alyssa Toledo², Kevin

Ramdas, $M D^{2}$, Meghana Shownkeen ${ }^{2}$, Luis Guada, $M D^{2}$, Dileep R Yavagal, $M D^{2}$

1 University of Miami Miller School of Medicine, Miami, Florida, USA;

${ }^{2}$ University of Miami, Miami, Florida, USA

Introduction: Thrombotic embolism is the most common cause of acute ischemic stroke, despite the embolic source having an unknown origin in $30-40 \%$ of cases. Mechanical thrombectomy has provided us with a method to directly retrieve the stroke-causing thrombi from ischemic stroke patients for analysis, outside of an autopsy. By collecting stroke-causing thrombi from known sources, we can then use novel RNA sequencing technology to directly study the composition of these clots. Using this RNA sequencing method may allow us to identify a marker that can identify the cause of cryptogenic embolism in the future. To our knowledge, there has not been any attempt at RNA extraction for profiling of stroke thrombi and we attempt to address its feasibility for future application.

Methods: This is a prospective IRB-approved study of acute middle cerebral artery ischemic stroke in patients receiving mechanical thrombectomy at a single academic center. Immediately after thrombi were retrieved by mechanical thrombectomy, they were place in RNA stabilization and storage solution (RNAlater). Each sample will be extracted for total RNA by the university's Human Genomics lab using a standard protocol. NanoDrop Microvolume Spectrophotometers and Fluorometer was used for RNA quantification. Thrombi weight was recorded. Pearson correlation was used for correlation analysis.

Results: From October 2016 to September 2017, five thrombi have been included in this study. Two thrombi were from patients with ipsilateral $>70 \%$ carotid stenosis, while the remaining three thrombi were from patients with atrial fibrillation. Average weight of the 5 thrombi was $25.8 \mathrm{mg}$ (range 12-36). Average extracted RNA concentration was $21.27 \mathrm{ng} / \mathrm{ul}$. There is a strong positive correlation between thrombi weight and extracted RNA concentration, $\mathrm{R}=0.78$. All of the thrombi-extracted RNA had an adequate laboratory RNA integrity score, used for RNA sequencing quality control.

Conclusions: This is the first study to confirm that RNA extraction from acute ischemic stroke thrombi is feasible. RNA sample may be used for future study to identify unique mRNA expression profiles of thrombectomy samples from known sources, laying the groundwork for the discovery of novel markers of stroke etiology.

Keywords: Thrombolytics, Stentretriever

Financial Disclosures: The authors had no disclosures.

Grant Support: SVIN Pilot Grant 
Platform Session I, Friday, November 10, 3:50-4:00 pm

\section{Endovascular Therapy and Ethnic Disparities in Stroke Outcomes}

Mehdi Bouslama, $M D^{1}$, Leticia C Rebello, $M D^{1}$, Diogo C Haussen, $M D^{1}$, Jonathan $A$ Grossberg, $M D^{1}$, Clara M Barreira, $M D^{1}$, Aaron M Anderson, MD', Samir $R$

Belagaje, $M D^{1}$, Nicolas A Bianchi, MD ${ }^{1}$, Michael R Frankel, $M D^{1}$, Raul G Nogueira, $M D^{1}$

${ }^{1}$ Emory University SOM/ Grady memorial Hospital, Atlanta, Georgia, USA

Introduction: Ethnic disparities in stroke are well described with higher incidence of disability and increased mortality in blacks versus whites. We sought to compare clinical outcomes between the latter ethnic groups after stroke endovascular therapy (ET).

Methods: Retrospective review of the prospectively acquired Grady Endovascular Stroke Therapy Outcomes Registry (GESTOR) between 9/2010-9/2015. Patients were dichotomized into 2 groups (1) Caucasians and (2) African-Americans, and matched for age, pretreatment glucose and baseline NIHSS. Baseline characteristics, procedural and outcome parameters were compared.

Results: Out of the 830 patients treated with endovascular therapy, 308 pairs of patients $(\mathrm{n}=616)$ underwent primary analysis. African Americans were younger $(p<0.01)$, had higher prevalence of hypertension $(p<0.01)$ and diabetes $(\mathrm{p}=0.04)$, and had higher ASPECTS $(\mathrm{p}=0.03)$ and shorter times to treatment $(\mathrm{p}=0.01)$. Blacks more frequently had Medicaid coverage and less private insurance $(29.6 \%$ vs $11.4 \%$ and $41.5 \%$ vs $60.3 \%$ respectively, $\mathrm{p}<0.01)$. The remaining baseline characteristics including baseline NIHSS and CT perfusion-derived ischemic core volumes were well balanced. There were no differences in the overall distribution of 90 -day $\mathrm{mRS}(\mathrm{p}=0.28)$, rates of successful reperfusion ( $84.7 \%$ vs $85.7 \%, p=0.91)$, good outcomes $(49.1 \%$ vs $44 \%, \mathrm{p}=0.24)$ or parenchymal hematomas $(6.5 \%$ vs $6.8 \%, \mathrm{p}=1.00)$. Blacks had lower 90 -day mortality rates $(18 \%$ vs $24.6 \%, \mathrm{p}=0.04)$ in univariate analysis which persisted as a non-significant trend after adjustment for potential confounders (OR 0.52 95\%CI 0.26-1.03, p = 0.06).

Conclusions: Despite unique baseline characteristics, African Americans treated with ET for LVO strokes have similar outcomes as Caucasians. Greater availability of ET may diminish the ethnic/racial disparities in stroke outcomes.

Keywords: Stroke, Endovascular Therapy

Financial Disclosures: The authors had no disclosures.

Grant Support: None. 
Platform Session I, Friday, November 10, 4:00-4:10 pm

\section{NCCT ASPECTS Modifies Intra-Arterial Treatment Effect in DAWN}

Parita Bhuva, M.D. ${ }^{1}$, Albert J Yoo, M.D., Ph.D. ${ }^{1}$, David S Liebeskind, M.D. ${ }^{2}$, Ashutosh J Jadhav, M.D., Ph.D. , Diogo C Haussen, M.D. , Ronald F Budzik, M.D. , Alain Bonafe, M.D. ', Dileep R Yavagal, M.D. ', Ricardo A Hanel, M.D. ${ }^{8}$, Marc Ribo, M.D. ${ }^{9}$, Christophe Cognard, M.D. ${ }^{10}$, Cathy Sila, M.D..$^{11}$, Ameer Hasan, D.O. ${ }^{12}$, Jeffrey $L$ Saver, M.D. ${ }^{2}$, Tudor G Jovin, M.D. ${ }^{3}$, Raul G Nogueira, M.D. ${ }^{4}$

${ }^{1}$ Texas Stroke Institute, Dallas-Fort Worth, Texas, USA; ${ }^{2}$ UCLA, Los Angeles, California, USA; ${ }^{3}$ University of Pittsburgh Medical Center, Pittsburg, Pennsylvania, USA; ${ }^{4}$ Grady Memorial Hospital, Emory University School of Medicine, Atlanta, Georgia, USA; ${ }^{5}$ OhioHealth Riverside Methodist Hospitals, Columbus, Ohio, USA; ${ }^{6}$ the Department of Neuroradiology, Hôpital Gui-deChauliac, Montpellier, France; ${ }^{7}$ University of Miami Miller School of MedicineJackson Memorial Hospital, Miami, Florida, USA; ${ }^{8}$ Baptist Jacksonville, Jacksonville, Florida, USA; ${ }^{9}$ Stroke Unit, Hospital Vall d'Hebrón, Barcelona, Spain; ${ }^{10}$ University Hospital of Toulouse, Toulouse, France; ${ }^{11}$ University Hospitals of Cleveland, Cleveland, Ohio, USA; ${ }^{12}$ Valley Baptist Medical Center, Department of Neuroscience, Harlingen, Texas, USA

Introduction: ASPECTS is widely used to assess infarct size and is established as a patient-selection tool in the traditional intra-arterial treatment (IAT) time window. Because the DAWN trial used advanced imaging, it is unclear if NCCT ASPECTS, which is more readily available, can be used for patient selection in the extended time window. We sought to characterize baseline ASPECTS in DAWN by imaging modality, and to assess whether ASPECTS modified IAT effects.

Methods: Core-lab ASPECTS was used for this analysis. ASPECTS score distribution was characterized in the entire population and in each clinicalimaging mismatch $(\mathrm{CIM})$ group: $A=$ infarct $\leq 21$, NIHSS $\geq 10$, age $\geq 80$; $B=$ infarct $\leq 31$, NIHSS $\geq 10$, age $<80$; $C=$ infarct $31-<51$, NIHSS $\geq 20$, age $<80$. We tested ASPECTS $\mathrm{x}$ treatment interaction for the entire ASPECTS scale and for dichotomized ASPECTS (0-6 [large] vs. 7-10 [small infarct]) for the endpoint of 90-day ordinal mRS.

Results: 197 DAWN patients were analyzed: 141(73\%) with NCCT ASPECTS, 77(40\%) with DWI ASPECTS, 21 patients with both. Median NCCT ASPECTS was 8(IQR 7-9) for the entire cohort; 8(8-9) for CIM A; 7.5(6-9) for CIM B; 7(5-8) for CIM C. Median DWI ASPECTS was 8(IQR 6-8) for the entire cohort; $8(8-9)$ for CIM A; 8(7-8) for CIM B; 5(5-5) for CIM C. There was significant modification of IAT effect by baseline NCCT ASPECTS for the entire scale (interaction $p=0.0265$ ); there was a trend for dichotomized ASPECTS (interaction $\mathrm{p}=0.0962$ ). IAT effect was more pronounced at higher NCCT ASPECTS scores. There was no significant modification of IAT effect for DWI ASPECTS for both the entire scale (interaction $\mathrm{p}=0.9387$ ) and dichotomized (interaction $\mathrm{p}=0.6239$ ). There was similar signal of treatment effect in each dichotomized ASPECTS group by DWI. 
Conclusions: Baseline NCCT ASPECTS modifies intra-arterial treatment effect in the DAWN cohort. Patients with higher NCCT ASPECTS appear to derive more benefit from IAT. No treatment interaction was seen for DWI ASPECTS.

Keywords: Acute Ischemic Stroke Intervention, Mechanical Thrombectomy, Endovascular Therapy, Imaging, Stroke

Financial Disclosures: The authors had no disclosures.

Grant Support: None. 
Platform Session I, Friday, November 10, 4:10-4:20 pm

\title{
Emergent Management of Tandem Lesions in Acute Ischemic Stroke: Analysis of the STRATIS Registry
}

\author{
Ashutosh P Jadhav, Asst Prof Neurol \& Neurol Surg', Osama O Zaidat, Medical \\ Director ${ }^{2}$, David S Liebeskind, Professor of Neurology ${ }^{3}$, Dileep R Yavagal, Assistant \\ Professor ${ }^{4}$, Diogo C Haussen, Assistant Professor ${ }^{5}$, Frank R Hellinger, Chief \\ Intervent Radiol', Reza Jahan, Professor Int Neuroradiol', Tudor G Jovin, Director \\ Stroke Inst ${ }^{1}$ \\ ${ }^{1}$ University of Pittsburgh Medical Center, Pittsburgh, Pennsylvania, USA; ${ }^{2} \mathrm{St}$. \\ Vincent Mercy Hospital, Toledo, Ohio, USA; ${ }^{3}$ University of California Los \\ Angeles Department of Neurology, Los Angeles, California, USA; ${ }^{4}$ University of \\ Miami Miller School of Medicine/Jackson Memorial Hospital, Miami, Florida, \\ USA; ${ }^{5}$ Emory University School of Medicine / Grady Memorial Hospital, \\ Atlanta, Georgia, USA; ${ }^{6}$ Florida Hospital, Orlando, Florida, USA; ${ }^{7}$ University of \\ California Los Angeles, Los Angeles, California, USA
}

Introduction: Large vessel occlusion is associated with poor outcomes in the absence of reperfusion, particularly in patients with tandem occlusions. While stent retriever thrombectomy is the standard of care for the intra-cranial occlusion, the best approach to the extra-cranial lesion remains unclear.

Methods: Data were analyzed from the STRATIS registry - a prospective, multicenter, observational, single-arm study of large vessel occlusion stroke patients treated with the Solitaire device (Medtronic, Irvine, Calif). A total of 984 patients were enrolled during the study. Univariate and multivariable logistic regression was used to assess relationship between outcome and procedural technique.

Results: Of 147 (14.9\%) patients treated within the STRATIS registry with tandem lesions, 80 patients were acutely stented and 67 patients were not acutely stented. There were no differences in age, CT ASPECT or IV-tPA use (76.3\% in stenting group vs $71.6 \%$ in non-stenting group, $\mathrm{p}=0.5)$; however, there was a slightly lower mean baseline NIHSS (16 vs $17.9, \mathrm{p}=0.07)$ and shorter mean onset to arterial puncture time (214 $\mathrm{min}$ vs $246 \mathrm{~min}, \mathrm{p}=0.04$ ) in the stenting group. Good outcomes (mRS 0-2 at 90 days) were higher in the stenting group $(68.5 \%$ vs $42.2 \%, \mathrm{p}=0.003)$ with no difference in mortality or symptomatic hemorrhage ( $0 \%$ vs $2.9 \%, \mathrm{p}=0.5)$. After adjustment for co-variates (age, baseline CT ASPECT, baseline mRS, baseline NIHSS, use of IV-tPA, onset to arterial puncture time, treating center volume size), stenting continued to be associated with superior outcomes. Within the stenting group, there was no difference in outcomes in patients undergoing stenting after thrombectomy compared to stenting before thrombectomy. In multivariate analysis, baseline NIHSS, CT ASPECT, and acute stenting were associated with good outcomes.

Conclusions: Acute stenting of an extra-cranial carotid stenosis during neurothrombectomy can be achieved without safety concerns. In a large real world experience, worse outcomes are observed in tandem lesions that are not acutely stented. 
Keywords: Carotid Stenting And Angioplasty, Acute Ischemic Stroke Intervention, Endovascular Therapy, Solitaire, Stentretriever

Financial Disclosures: The authors had no disclosures.

Grant Support: None. 
Platform Session I, Friday, November 10, 4:20-4:30 pm

\section{FAST-IA: Flat-Detector CT Assessment in Stroke to Reduce Times to Intra-Arterial Treatment}

Mehdi Bouslama, $M D^{1}$, Diogo C Haussen, $M D^{1}$, Jonathan A Grossberg, $M D^{1}$, Clara M Barreira, MD', Samir R Belagaje, $M D^{1}$, Nicolas A Bianchi, $M D^{1}$, Aaron $M$

Anderson, $M D^{1}$, Michael $R$ Frankel, $M D^{1}$, Raul G Nogueira, $M D^{1}$

${ }^{1}$ Emory University SOM/ Grady Memorial Hospital, Atlanta, Georgia, USA

Introduction: Time to treatment is one of the strongest determinants of outcomes in large vessel occlusion (LVO) stroke with the chances of good outcomes declining on average $10-15 \%$ for every 30 -minute delay in reperfusion. Bypassing the emergency department (ED) and the CT suite with direct transport to the neuroangiography suite for assessment through the use of Flat Detector (FD) CT imaging may safely shorten reperfusion times.

Methods: A single-center retrospective review of consecutive patients with anterior circulation LVO strokes transferred to our facility for consideration for endovascular therapy (ET) from 5/2016-4/2017. Those with basilar strokes and/or presenting to the ED were excluded. Patients were categorized into 2 groups: (1) Flat-Detector CT Assessment in Stroke to Reduce Times to Intra-Arterial Treatment (FAST-IA), including those that were transferred directly to the suite and underwent FD-CTA imaging; and (2) a control group comprising those that had the standard protocol including CT +/- CTA and CTP. Baseline characteristics, time metrics and outcomes were compared.

Results: Out of 238 patients that underwent ET, 120 patients fit inclusion criteria, of which 28 (23\%) were in the FAST-IA group and $92(77 \%)$ in the control group. Demographics, comorbidities as well as stroke severity were well balanced. FAST-IA patients had significantly shorter median door to puncture times (38.5 mins [30-45.5] vs 59[48-79], $\mathrm{p}<0.001$ ), door to reperfusion times (74[57.5-87.8] vs $108.5[88.5-153.3], \mathrm{p}=0.03)$ and picture to puncture times (14[12.5-19] vs 37.5[33.5-51], $\mathrm{p}<0.001)$. There were no differences between groups in rates of successful reperfusion (mTICI 2b-3) (96.4\% for Fast-IA vs $98.9 \%, \mathrm{p}=0.42)$, type-2 parenchymal hematomas $(8.2 \%$ vs $6.7 \%, \mathrm{p}=1.00)$, 90 -day good outcome $(35.3 \%$ vs $42.6 \%, \mathrm{p}=0.58)$ and mortality $(29.4 \%$ vs $22.1 \%, \mathrm{p}=0.53)$.

Conclusions: Directly transferring patients to angio and using FD-CT imaging to determine eligibility for stroke ET safe, feasible and results in significant reduction in door to needle, door to reperfusion and picture to puncture times. Future studies are warranted.

Keywords: Stroke, Imaging, Endovascular Therapy

Financial Disclosures: The authors had no disclosures.

Grant Support: None. 
Platform Session II, Saturday, November 11, 9:00-9:10 am

\section{Optimized Hyaluronan-Based Hydrogels Selectively Control Survival and Differentiation of Human Stem Cells Transplanted in Stroke}

Pouria Moshayedi, $M D, P h D^{1}$, Lina Nih, $P h D^{2}$, Irene L Llorente, $P h D^{3}$, Andrew $R$ Berg, $B S c^{3}$, Jessica Cinkornpumin, $M S c^{4}$, William Lowry, $P h D^{4}$, Tatiana Segura, $P h D^{2}$, S. Thomas Carmichael, $M D, P h D^{3}$

'Department of Neurology, University of Pittsburgh Medical Center, Pittsburgh, Pennsylvania, USA; ${ }^{2}$ Department of Chem and Biomol

Engineering, University of California Los Angeles, Los Angeles, California, USA; ${ }^{3}$ Department of Neurology, University of California Los Angeles, Los Angeles, California, USA; ${ }^{4}$ Department of Mol Cell and Dev Biology, University of California Los Angeles, Los Angeles, California, USA

Introduction: Stroke is among the leading causes of disability in the western population and majority of stroke survivors live with permanent disabilities. Stem cells have been used for almost two decades to promote repair and recovery in stroke patients, but their success has been limited by their suboptimal survival. This highlights importance of utilizing bioengineering and biophysics tools to design bio-scaffolds and provide cells with adhesive and growth factors, protect transplanted cells from host ischemic and inflammatory insults and to improve stem cell differentiation and integration.

Methods: We used biophysical methods to study effects of material stiffness on foreign body response. We then studied hyaluronic acid (HA)-based self-polymerizing hydrogel as a platform for adhesion of structural motifs and a depot release for growth factors. An iterative approach enabled us to optimize the complex combination of mechanical, biochemical and biological properties of an HA cell bio-scaffold.

Results: Adjusting the stiffness of hydrogel to mimic brain stiffness diminished inflammatory response in the host astrocytes and microglia, and it decreased foreign body reaction in the brain. We were then able to promote vascularization by making hydrogel crosslinker sensitive to tissue matrix metalloproteinases. In the last step of optimization a Design of Experiment approach was utilized to systematically change candidate adhesion motifs and growth factors to optimize stem cell survival or proliferation. We finally tested the optimized HA hydrogel in vivo and showed it promoted survival of encapsulated human neural progenitor cells (iPS-NPCs) after transplantation into the stroke core. It also deferentially tuned transplanted cell fate towards glial, neuronal or immature/progenitor fates. The water content of HA hydrogel made it trackable in vivo with MRI.

Conclusions: A mechanically, biochemically and biologically optimized hydrogel can serve as a therapeutic adjunct in stem cell therapy through selective control of stem cell survival and differentiation in vivo.

Keywords: Ischemic Stroke, Basic Sciences, Stem Cell Therapy

Financial Disclosures: The authors had no disclosures.

Grant Support: CIRM (RT2-01881), NIH (R01NS079691) 
Platform Session II, Saturday, November 11, 9:10-9:20 am

\section{Ineligibility for Endovascular Treatment in Large Vessel Occlusions within Time Window: Characteristics and Outcomes}

Aashish Anand, $M D^{1}$, Sowmya Rao, $P A^{1}$, Naregnia Pierre-Louis, $M D^{1}$, Benny Kim, $M D^{1}$, Alexander Venizelos, $M D^{1}$, Paul Hansen, $M D^{1}$, Norman Ajiboye, $M D^{1}$, Ryan Gianatasio, $M D^{1}$, Parita Bhuva, $M D^{1}$, Albert Yoo, $M D^{1}$

${ }^{1}$ Texas Stroke Institute, Plano, Texas, USA

Introduction: There are limited data on large vessel occlusion (LVO) patients who remain ineligible for intra-arterial treatment (IAT) despite presentation within the therapeutic time window. Our study evaluates reasons, characteristics and outcomes for these LVO patients.

Methods: Clinical data of all LVO patients during a 10-month period was collected from a prospective regional tele-stroke registry. Prevalence, clinical and outcome data of eligible and ineligible LVO patients within the 8-hour time window is compared and presented.

Results: $42 \%$ (69) of 166 LVO patients presenting within 8 hours were considered ineligible for thrombectomy. These included ASPECT score $<6$ on CT/ large core infarct volume on MRI in 43, low NIH stroke scale in 15, refusal by family in 4 , poor pre-morbid functional status in 3 , anticipated difficult trans-arterial access in 2 and hemorrhagic transformation after alteplase in 2 patients. The ineligible LVOs were more likely to first present to an "out of network" hospital $(\mathrm{p}=0.04)$, had lower rates of treatment with alteplase $(\mathrm{p}=0.04)$, and were less likely to receive advanced imaging $(\mathrm{p}=0.003)$. There was no significant difference in sex $(\mathrm{p}=0.4)$, mean age $(\mathrm{p}=0.2)$, initial NIHSS $(\mathrm{p}=0.9)$, time from last normal to presentation at comprehensive stroke center (CSC) $(p=0.1)$, direct presentation to a CSC $(p=0.06)$, higher clot burden on vascular imaging $(\mathrm{p}=0.3)$ and pre-morbid vascular risk factors. There was a non-significant trend towards poor modified Rankin score $(\mathrm{mRS})$ at discharge among ineligible LVOs $(\mathrm{p}=0.06)$. Among ineligible LVO patients, poor ASPECT score was associated with poor $\mathrm{mRS}$ at discharge $(\mathrm{p}=$ 0.002). In linear regression analysis, ineligible LVOs were independently associated with "out of network" presentation $(\mathrm{p}=0.03)$ and poor $\mathrm{mRS}$ at discharge $(\mathrm{p}=0.03)$.

Conclusions: A significant percentage of LVOs remain ineligible for endovascular treatment despite presentation within 8 hours. Poor ASPECTS accounts for the majority of these cases.

Keywords: Stroke, TICI, Intra-Arterial Therapy.

Financial Disclosures: The authors had no disclosures.

Grant Support: None. 
Platform Session II, Saturday, November 11, 9:20-9:30 am

\title{
Comparison of Outcomes with Thrombectomy Devices for Treatment of Acute Stroke: A Bayesian Network Meta- Analysis
}

\author{
Hamidreza Saber, $M D, M P H^{1}$, Sandra Narayanan, $M D^{2}$
}

${ }^{1}$ Wayne State University School of Medicine, Detroit, Michigan, USA; ${ }^{2}$ Wayne State University School of Medicine/Detroit Medical Center, Detroit, Michigan, USA

Introduction: Mechanical thrombectomy (MT) is increasingly used for the treatment of emergent large vessel occlusion (ELVO). Yet, randomized clinical trial (RCT) level data is limited on differences in efficacy or safety of MT devices. We aimed to conduct a network meta-analysis (NMA) that enables comparison of MT devices across trials.

Methods: A search was performed to identify RCTs that used thrombectomy devices (Merci, Trevo, Solitaire, Aspiration) in one or both arms for ELVO. The efficacy outcome was 90-day functional independence (mRS $0-2$ ), and safety outcomes were 90-day catastrophic outcome (mRS 5-6) and symptomatic intracranial hemorrhage (sICH). A fixed-effect Bayesian NMA was performed to calculate the odd-ratios (ORs) and their corresponding 95\% credible-interval (CrI). The rank-probability of each device for different outcomes were reported using the surface under the cumulative ranking curve.

Results: Six relevant RCTs were included (SWIFT, TREVO2, EXTEND-IA, SWIFT-PRIME, REVASCAT, THERAPY), comprising of 871 patients with ELVO, of whom 472 were in the Solitaire vs. medical-only trials, 108 in the Aspiration vs. medical-only trial, 178 in the Trevo vs. Merci trial, and 113 in the Solitaire vs. Merci trial. The NMA indicated that with medical-only arm as the reference, Trevo had the greatest functional independence (OR 4.26, 95\% CrI, 1.35-12.88; top-rank probability 93\%) followed by Solitaire device (OR 2.49, 95\% CrI, 1.7-3.74; top-rank probability 70\%). Solitaire and Aspiration devices had the greatest top-rank probability with respect to low sICH and catastrophic outcomes ( $76 \%$ and $91 \%$, respectively), but without significant differences from each other (OR 0.72, 95\% CrI, 0.23-2.05). Sensitivity analysis incorporating preliminary results from DAWN and ASTER trials did not alter these ranks.

Conclusions: These findings suggest that Trevo and Solitaire devices are associated with a greater likelihood of functional independence, whereas Solitaire and Aspiration devices appear to be safer than others with lower rates of sICH and catastrophic outcomes.

Keywords: Mechanical Thrombectomy, Ischemic Stroke, Acute Ischemic Stroke Intervention

Financial Disclosures: The authors had no disclosures.

Grant Support: None. 
Platform Session II, Saturday, November 11, 9:30-9:40 am

\title{
Reperfusion with ACE64 and ACE68 in the Treatment of Acute Ischemic Stroke. The PROMISE Registry
}

\author{
Peter Schramm, Doctor ${ }^{1}$, Pedro Navia, Neuroradiologist ${ }^{2}$, Rosario Papa, Doctor ${ }^{3}$, \\ Joaquin Z Parra, Doctor ${ }^{4}$, Alejandro T Weitz, Doctor ${ }^{5}$, Werner Weber, Doctor ${ }^{6}$, Jens \\ Fiehler, Doctor ${ }^{7}$, Patrik Michel, Doctor ${ }^{8}$, Vitor M Pereira, Doctor ${ }^{9}$, Timo Krings, \\ Doctor $^{9}$, Laurent Pierot, Doctor ${ }^{10}$, Jan Gralla, Doctor ${ }^{11}$, Paola Santalucia, Doctor ${ }^{12}$, \\ Tjoen H Lo, Doctor ${ }^{13}$ \\ 'University of Lubeck, Luebeck, Germany; ${ }^{2}$ Hospital Universitario Donostia, \\ San Sebastian, Guipúzcoa, Spain; ${ }^{3}$ A.O.U. Policlinico, Messina, Italy; ${ }^{4}$ Hospital \\ Clínico Universitario Virgen de la Arrixaca, Murcia, Spain; ${ }^{5}$ Vall d'Hebron \\ Hospital, Barcelona, Spain; ${ }^{6}$ Universitätsklinikum Knappschaftskrankenhaus \\ Bochum, Bochum, Germany; ${ }^{7}$ Universitätsklinikum Hamburg-Eppendorf, \\ Hamburg, Germany; ${ }^{8}$ Centre Hospitalier Universitaire Vaudoi, Lausanne, \\ Switzerland; ${ }^{9}$ University of Toronto, Toronto Western Hospital, Toronto, \\ Canada; ${ }^{10}$ Hôpital Maison Blanche, Reims Cedex, France; ${ }^{11}$ Inselspital \\ Neuroradiology, Bern, Switzerland; ${ }^{12}$ Fondazione IRCCS Ca' Granda Ospedale \\ Maggiore Policlinico, Milan, Italy; ${ }^{13}$ University Medical Center Utrecht, Utrecht, \\ Netherlands
}

Introduction: The aim of PROMISE registry is to evaluate safety and effectiveness of Penumbra System with the latest generation of ACE Reperfusion Catheters in a real-world population with acute ischemic stroke from anterior circulation large vessel occlusion (LVO), treated with the ADAPT technique in routine clinical practice. Safe and complete recanalization is important to reduce clot burden and determines the functional prognosis of patients with LVO acute ischemic stroke.

Methods: The PROMISE is a prospective, single-arm and multicenter registry, evaluating reperfusion with ACE64 and ACE68 catheters across 20 European centers. The primary endpoints included success in angiographic revascularization (TICI 2b-3) and clinical independence (mRS 0-2) at 90 days. Secondary endpoints included safety events, functional improvement at 7-10 days, procedural metrics and quality of life. Criteria for inclusion included the presentation of anterior circulation LVO within 6 hours of ictus, NIHSS $\geq 2$, CT-ASPECTS $\geq 6$ or MR DWI ASPECTS $\geq 5$ and wherein intervention proceeded with ADAPT as frontline. mTICI is adjudicated by a core laboratory. Safety endpoints are adjudicated by clinical events committee.

Results: A total of 201 patients were enrolled for evaluation of this interim analysis in the PROMISE registry. The mean (SD) age is 71.2 (12.4). The mean (SD) baseline NIHSS scores of 15.4 (5.9). The pre-stroke mRS score was within $0-2$ range, with $25.5 \%$ of subjects with $\mathrm{mRS}$ score greater than 0 . The mean (SD) baseline CT ASPECT score was 9.1(1.4). Occlusion vessel location observed $8.5 \%(17 / 201)$ of patients with ICA occlusion, $12.9 \%$ (26/201) with Carotid T, 67.7\% (136/201) with M1 and 10.9\% (22/201) with M2 respectively. Prior to the endovascular procedure, IV rtPA was administered in $61.7 \%(124 / 201)$ of patients. 
Conclusions: Data from the PROMISE registry will assess the safety and effectiveness of the Penumbra System with the novel ACE Aspiration Catheters using ADAPT as the frontline treatment.

Keywords: Acute Ischemic Stroke Intervention, Endovascular Therapy, Recanalization, Penumbra, TICI

Financial Disclosures: The authors had no disclosures.

Grant Support: None. 


\section{Posterior Circulation Acute Stroke Prognosis Early CT Score in Selecting Patients for Posterior Circulations Recanalization}

Kasra Khatibi, $M D^{1}$, May Nour, $M D, P h D^{1}$, Satoshi Tateshima, $M D^{1}$, Reza Jahan, $M D^{1}$, Gary Duckwiler, $M D^{1}$, Jeffrey Saver, $M D^{1}$, Viktor Szeder, $M D, P h D^{1}$

${ }^{1}$ University of California Los Angeles, Los Angeles, California, USA

Introduction: Basilar artery occlusion (BAO) has a high level of mortality and disability, for whom benefit of recanalization has been established. However, unlike anterior circulation stroke no well-established clinical or radiographic criteria is available to identify which patients will benefit from endovascular recanalization. Our aim was to evaluate posterior circulation Acute Stroke Prognosis Early CT score (pc-ASPECT) applied to MRI and CT as a prognostic factor in the patients who had undergone posterior circulation endovascular recanalization at our institution and compare to their clinical outcome.

Methods: All patients who had undergone endovascular recanalization of posterior circulation between years of 2002 and 2017 were included. pcASPECT score and the anatomical location of early ischemia was calculated/ recorded for the immediately prior to intervention MRI or CT for each patient. The pc-ASPECT score and location of the ischemia was compared to the modified ranking score (MRS) at 90 days using a Spearman Correlation Coefficient. The groups were later dichotomized to identify sensitivity of the radiographic findings for identification of unfavorable outcome.

Results: 19 patients with MRI and 8 patients with CT were identified and included in the study. The correlation coefficient of pc-ASPECT compared to MRS was -0.78 and +0.3 for MRI and CT group respectively. The highest anatomical correlation with the MRS was ischemic burden in the midbrain (0.65). pc-ASPECT score was significantly higher in the patients with favorable neurological outcome with MRS $\leq 2(\mathrm{p}=0.0001)$. pc-ASPECT score $<7$ sensitivity for unfavorable outcome was $100 \%$ with specificity $87.5 \%$.

Conclusions: pc-ASPECT score applied to MRI could play a role in selecting patients with basilar artery occlusion that would benefit from endovascular recanalization.

Keywords: Acute Ischemic Stroke Intervention, MRI, Endovascular, Recanalization

Financial Disclosures: The authors had no disclosures.

Grant Support: None. 
Platform Session II, Saturday, November 11, 9:50-10:00 am

\title{
Egyptian Stroke Network, Current Problems and Call for Action, Alexandria Prototype
}

\author{
Ossama y Mansour, $M D, P h D, F N R^{1}$, Ashraf Abdou, $M D^{2}$, Farouk Talaat, $M D^{2}$, \\ Hazem Marouf, $M D^{2}$, Ismail Ramadan, $M D^{2}$, Sameh Saeed, $M D^{2}$, Mohamed \\ Hamdy, $M D^{2}$, Jiadaa Mekky, $M D^{2}$, Horria Saeed, $M D^{2}$, Ahmed Deef, $M D^{2}$, Aymen \\ Ezeldeen, $M D^{2}$, Amer Elfatatry, $M D^{2}$, Mervat Hamed, $M D^{2}$
}

${ }^{1}$ Alexandria university, Stroke and interventional neurology center, Alexandria, Egypt; ${ }^{2}$ Alexandria University, Alexandria, Egypt

Introduction: Stroke is one of the major public health challenges in middle-income countries. Egypt is the most populated nation in the Middle East, according to recent estimates, the overall prevalence rate of stroke is high with a crude prevalence rate of 963/100000 inhabitants. In spite of disease burden, yet there is a huge evidence practice gap and clearly behind the milestones in the fight against stroke, which is the leading cause of death and disability.

Aims: The present article discusses the evolution of stroke care in Egypt with focus on the results of the Applying policy of the Egyptian stroke Network in Alexandria as a prototype for all Egypt.

Methods: We describe the main characteristics of stroke care before 2010; a pilot study in a Alexandria city between 2008 and 2010, the Egyptian Stroke Project initiative Alexandria Prototype; and the 2017 National Stroke Policy Act.

Results: The National Stroke Project was followed by a major increased on the number of stroke center in the Alexandria City. The key elements of the 2017 National Stroke Policy Act included: definition of the requirements and levels of stroke centers; improved reimbursement for stroke care; promotion of stroke telemedicine; definition of the Line of Stroke Care (to integrate available resources and other health programs); increased funding for stroke rehabilitation; funding for training of healthcare professionals and initiatives to increase awareness about stroke within the population, Improving Prehospital Triage system, Including Software for decreasing time of diagnosis and dispatch.

Conclusions: The evolution of stroke care in Alexandria over the last few years is a pathway that exemplifies the challenges that middle-income countries have to face in order to improve stroke prevention, treatment and rehabilitation. The reported Alexandria experience can be extrapolated to understand the past, present, and future of stroke care in middle-income countries.

Keywords: Acute Stroke, New Innovation, Thrombolytics, Acute Ischemic Stroke Intervention, Door To Needle

Financial Disclosures: The authors had no disclosures.

Grant Support: None. 